
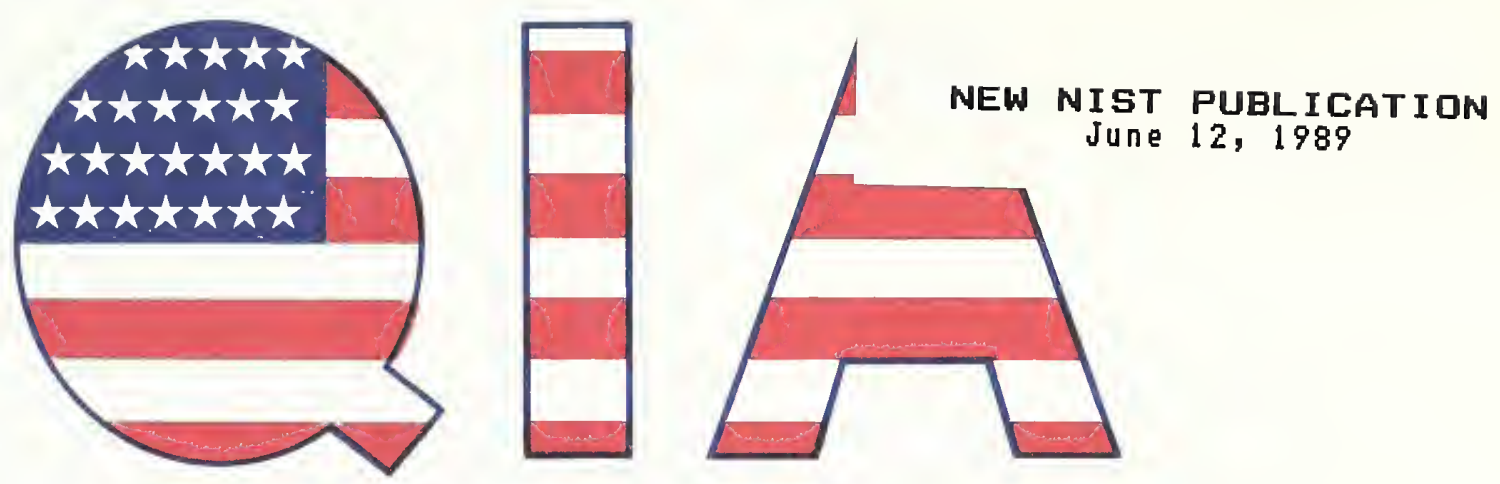

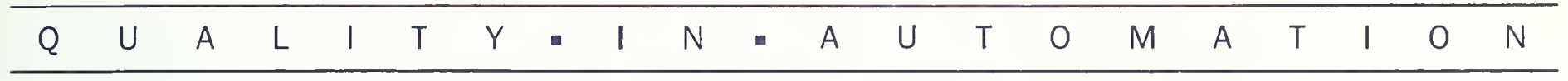

\title{
Progress Report of the Quality in Automation Project for FY88
}

U.S. DEPARTMENT OF COMMERCE National Institute of Standards and Technology

Gaithersburg, Maryland 20899

NISTIR 89-4045

Edited by

C. Denver Lovett

April 1989

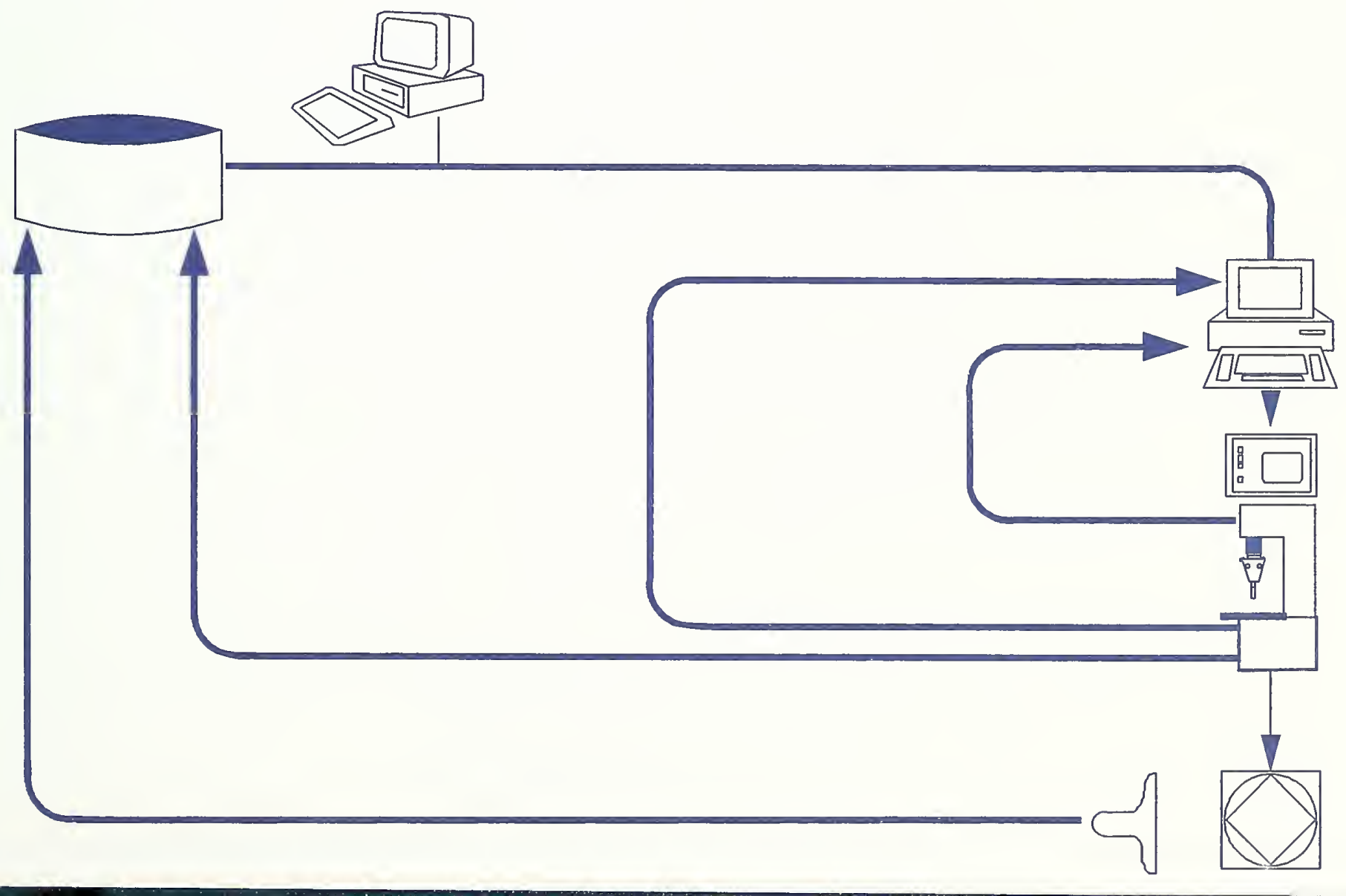





\section{NISTIR 89-4045}

\section{Progress Report of the Quality in Automation Project for FY88}

Edited by

C. Denver Lovett

U.S. DEPARTMENT OF COMMERCE

National Institute of Standards and Technology

Gaithersburg, MD 20899

April 1989

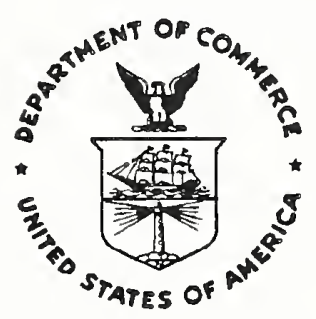

U.S. DEPARTMENT OF COMMERCE

Robert Mosbacher, Secretary

NATIONAL INSTITUTE OF STANDARDS

AND TECHNOLOGY

Raymond G. Kammer, Acting Director 



\section{DISCLAIMER}

Commercial equipment, instruments, or materials are identified in this report in order to specify adequately certain experimental procedures. In no case, does such identification imply recommendation or endorsement by the National Institute of Standards and Technology, nor does it imply that the material or equipment identified is necessarily the best available for the purpose.

\section{ACKNOWLEDGMENTS}

The funding provided by the Navy's Manufacturing Technology Program and DOE's Precision Flexible Manufacturing Project has made this research project possible. We appreciate their support. Also we are especially grateful to Dr. Ronald Miskell of Martin Marietta, Energy Systems Division. Dr. Miskell's suggestions and guidance have provided reassurance and a solid basis for continuing research.

We also acknowledge Dr. A. Donmez, Dr. R. Hocken, Dr. T. Hopp, Dr. T. Kramer, D. Capparrelli, J. McMahon, N. Gilman, and S. Osella for their frequent technical contributions and V. Gagne and C. Keyser for their assistance in the preparation of the manuscript. 



\begin{abstract}
This document describes a quality control architecture that uses real-time sensing, deterministic metrology methods, machine tool characterization, process-intermittent gauging, and process certification to control automated machining processes. The architecture was developed for the quality in automation (QIA) project which has the two-fold objective of reducing the reliance on traditional post-process inspection methods and moving toward greater reliance on in-process verification. The QIA project is developing a computer-based system that includes realtime sensors, a quality database, quality monitors, quality controllers, an inspection station, and a communication network, which allows data to flow among components of the quality system. The quality architecture includes control loops such as post-processing characterization, preprocessing characterization, process-intermittent gauging and real-time sensing (see Fig. 2.7). The sensors for temperature, force, and ultrasonics may result in a command to the machine tool controller to alter its pre-programmed position, to change its manipulative variables (speed and feed), and to modify the NC code. The corrective action will depend on the time varying nature of the errors and the predictions derived from the analysis models. A strategy for allocating the corrective action to a real-time error corrector or a software module that revises the process plan and the NC code is being developed. The machining of standard workpieces (NAS and modified BAS) and machine tool characterization serves as a major component in certifying the machining process.
\end{abstract}





\section{TABLE OF CONTENTS}

Page

1. INTRODUCTION

2. AN ARCHITECTURE FOR QUALITY CONTROL IN THE QIA PROJECT . . . . 5

2.1 ARCHITECTURE OVERVIEW . . . . . . . . . . . . . . 5

QIA Scenario. . . . . . . . . . . . . . . 5

2.2 CONTROL ARCHITECTURE. . . . . . . . . . . . . . . . . . 8

2.2.1 PRE-PROCESS CHARACTERIZATION. . . . . . . . . . . 10

2.2.2 REAL-TIME SENSING . . . . . . . . . . . . . . . . . 12

Thermal State of the Machine. . . . . . . . . . . . . 14

Vibration. . . . . . . . . . . . . . . . . 14

Force . . . . . . . . . . . . . . . . . . . . 15

Ultrasonics . . . . . . . . . . . . . . . . . 16

2.2.3 PROCESS-INTERMITTENT GAUGING. . . . . . . . . . . . . . 19

Part Dimensions and Form. . . . . . . . . . . . . . . 19

Part Orientation. . . . . . . . . . . . . . 22

Tool Setting. . . . . . . . . . . . . . . 22

Surface Texture . . . . . . . . . . . . . . . 22

2.2 .4 POST-PROCESS GAUGING. . . . . . . . . . . . . . 24

2.2 .5 GENERAL ...................... . . . 27

2.3 DATA REQUIREMENTS FOR AUTOMATED MANUFACTURING . . . . . 29

2.3.1 OVERVIEW OF DATA REQUIREMENTS . . . . . . . . . . . . . 29

2.3.2 THE CAD CLUSTER . . . . . . . . . . . . . . . . 29

2.3.3 THE PROCESS PLANNING CLUSTER. . . . . . . . . . . . . . 30

2.3.4 THE PART PROGRAMMING CLUSTER. . . . . . . . . . . . . . 31

2.3 .5 THE REAL-TIME SUPPORT CLUSTER . . . . . . . . . . . . . 32

2.3.6 THE CERTIFICATION CLUSTER . . . . . . . . . . . . . . 34

2.4 PROGRAMMING LANGUAGE ENVIRONMENT. . . . . . . . . . 34

2.4.1 OFF-LINE MODE . . . . . . . . . . . . . . . . . . 36

2.4.2 REAL-TIME MODE. . . . . . . . . . . . . . . . 38

2.5 INTEGRATION WITH THE AMRF............ 40

3. MACHINE TOOL CHARACTERIZATION . . . . . . . . . . . . 48

3.1 OVERVIEW. . . . . . . . . . . . . . . . . 48

3.2 MODEL FOR ERROR COMPENSATION. . . . . . . . . . . . 49

3.3 EXPERIMENTAL BASIS FOR ESTIMATING POSITIONING ERRORS. 50

3.3.1 DATA ACQUISITION REQUIREMENTS . . . . . . . . . . . 50

3.3.2 DATA ACQUISITION SYSTEM . . . . . . . . . . . . . . . 52

3.3.3 THERMOCOUPLE INSTALLATION . . . . . . . . . . . . . . 52

3.3.4 DATA LOGGING. . . . . . . . . . . . . . . . . . . . . . 56

3.3.5 MEASUREMENT OF POSITIONING ERRORS . . . . . . . . . . . 65

3.3 .6 DATA ANALYSIS . . . . . . . . . . . . . . . . . . . . . 72 
4. TEST PARTS AND INSPECTION RESUlTS. . . . . . . . . . . . . 74
4.1 GENERAL
74
4.2 TEST PART SELECTION ................ . . 74
4.3 FIXTURES AND BLANK PREPARATION. . . . . . . . . . . 76
4.4 NC CODE FOR FIRST TEST PART AND INSPECTION. . . . . . . 76

5. REAL-TIME ERROR CORRECTOR AND PROCESS INTERMITTENT PROBING . . 83

5.1 GENERAL . . . . . . . . . . . . . . . . . . 83

5.2 CONCEPTUAL DESIGN OF RTEC . . . . . . . . . . . . . 83

5.3 IMPLEMENTATION. . . . . . . . . . . . . . . 85

5.4 TESTING . . . . . . . . . . . . . . . . . 86

6. QUALITY ARCHITECTURE NETWORK . . . . . . . . . . . . . 87

6.1 GENERAL . . . . . . . . . . . . . . 87

6.2 ETHERNET FEATURES . . . . . . . . . . . . . . . . 87

6.3 IMPLEMENTATION. . . . . . . . . . . . . . . . . . . . . 88

7. ULTRASONIC SENSOR . . . . . . . . . . . . . . . . . . . . 89

7.1 GENERAL . . . . . . . . . . . . . . . . . . . . . . . 89

7.2 CONCEPTUAL DESIGN . . . . . . . . . . . . . . . . . . 89

7.3 IMPLEMENTATION AND TEST .............. . . 90

7.4 FUTURE PLANS. . . . . . . . . . . . . . . . . . . . 92

8. VISUALIZATION OF INSPECTION RESULTS . . . . . . . . . . . . 93

8.1 GENERAL ................... . . . . 93

8.2 DESIGN CONSIDERATIONS . . . . . . . . . . . . . . . . . 93

8.3 IMPLEMENTATiON AND TESTING. . . . . . . . . . . . . . 94

9. REFERENCES . . . . . . . . . . . . . . . . . 95 


\section{LIST OF TABLES}

Page

TABLE 2.1 AMRF ARCHITECTURE: LEVEL STRUCTURE AND FUNCTIONS. . 42

TABLE 2.2 SUPPORTING ELEMENTS FOR QIA PROJECT AT CELL LEVEL . 44

TABLE 3.1 THERMOCOUPLE LOCATIONS ON MONARCH VMC . . . . . . . . 58

TABLE 3.2 THERMOCOUPLE LOCATIONS ON WHITE-SUNSTRAND HWS . . . 61

TABLE 3.3 THERMOCOUPLE LOCATIONS ON MONARCH METALIST. . . . . . 63

TABLE 3.4 MEASUREMENTS REQUIRED FOR POSITIONING ERRORS. . . . . 70 
. 


\section{LIST OF FIGURES}

$\underline{\text { Page }}$

FIG. 2.1

FIG. 2.2

FIG. 2.3

FIG. 2.4

FIG. 2.5

FIG. 2.6

FIG. 2.7

FIG. 2.8

FIG. 2.9

FIG. 3.1

FIG. 3.2

FIG. 3.3

FIG. 3.4

FIG. 3.5

FIG. $3.6 \mathrm{a}$

FIG. $3.6 \mathrm{~b}$

FIG. 3.7

FIG. 3.8

FIG. $3.9 a$

FIG. $3.9 b$

FIG. $3.10 \mathrm{a}$

FIG. $3.10 \mathrm{~b}$

FIG. 3.11

FIG. 4.1

FIG. 4.2

FIG. 4.3

FIG. 4.4

FIG. 4.5

FIG. 4.6

FIG. 5.1

FIG. 7.1
QIA Project Overview . . . . . . . . . . . . . . . . 9

Control Loop for Pre-Process Characterization. . . . . . 11

Control Loop for Real-Time Sensing . . . . . . . . . . 13

Tool Force as an Indicator of Wear . . . . . . . . . . . 17

Control Loop for Process-Intermittent Gauging. . . . . . 20

Control Loop for Post-Process Gauging. . . . . . . . . . 26

Overall Control Loop Architecture for QIA. . . . . . . . 28

Expanded AMRF Control Hierarchy. . . . . . . . . . . . . 41

Network Communication Plan . . . . . . . . . . . . . . 46

Event Sequence for Data Acquisition . . . . . . . . 51

Data Acquisition System. . . . . . . . . . . . . . . 53

Thermocouple Fabrication Sequence. . . . . . . . . . . 54

Typical Thermocouple Installation. . . . . . . . . . . . 55

Thermocouple Locations for Vertical Machining Center . 57

Thermocouple Locations for Horizontal Machining Center . 59

Thermocouple Locations for Horizontal Machining Center: Z-axis Drive. . . . . . . . . . . . . . . . . . 60

Thermocouple Locations for Turning Center. . . . . . . . 62

Temperature Time History for Vertical Machining Center:

Machining Operations for Three Different Parts . . . . 64

Temperature Time History for Vertical Machining Center: Warm-Up Period (Spingle Speed is $1375 \mathrm{rpm}$ ) . . . . . . 66

Temperature Time History for Vertical Machining Center: Cool-Down Period. . . . . . . . . . . . . . . . . . 67

Temperature Time History for Vertical Machining Center: Warm-Up Period (Spindle Speed is $2500 \mathrm{rpm}$ ) . . . . . . 68

Temperature Time History for Vertical Machining Center: Cool-Down Period. . . . . . . . . . . . . . . . 69

Nomenclature for the Six Degrees-of-Freedom for Rigid Body Motion along an Axis. . . . . . . . . . . 71

Test Parts... . . . . . . . . . . . . 75

Six Probing Points for Larger Square. . . . . . . . . . 77

Measured Values of Length, Squareness and Parallelism for Larger Square. . . . . . . . . . . . . . . 78

The Radius Measurements at 12 Points . . . . . . . . . 79

Measured Values of Length, Squareness and Parallelism for Small Square. . . . . . . . . . . . . . . . . . 80

Six Probing Points for Small Square. . . . . . . . . 81

Machine Tool Quality Improvement Strategy. . . . . . . . 84

Relative Echo Amplitude. . . . . . . . . . . . . 91 


\section{INTRODUCTION}

C. Denver Lovett

The objectives of this report are to describe the Quality in Automation (QIA) Project goals, to report some of the accomplishments toward meeting those goals, and to give enough background information on the QIA project so that its goals and the related accomplishments are appreciated.

Today's innovations in manufacturing technology are driven by demands to shorten product cycle time and to maintain a consistent high level of product quality in an unattended manufacturing environment. These demands are sometimes generated by high accuracy requirements of our defense industry and at other times by competitive market forces on industry to produce high quality products with a short lead-time. One approach to meeting these demands is to implement a quality system that can monitor, measure and control significant parameters of the manufacturing process and of the machine tool. The National Institute of Standards and Technology (NIST) Center for Manufacturing Engineering has a QIA program that takes this approach to quality. This program is funded by the Navy in support of the quality efforts of the Automated Manufacturing Research Facility (AMRF), by the Department of Energy (DOE) to support the quality efforts of their Precision Flexible Manufacturing System (PFMS), and by direct NIST funds.

The QIA project has two sets of goals to satisfy, those of the AMRF/Navy program and those of the DOE's PFMS program. A mutual goal of both programs is to use sensor technology and deterministic metrology methods to verify that the machining processes can maintain their accuracy under various temperature conditions of the machine tool.

The ultimate goal of PFMS is to implement a quality control system that assures a high level of product quality, without totally depending on the traditional product inspection process to provide that quality assurance. However, during the initial operation, the PFMS quality assurance will focus on three components: (1) in-process quality 
assurance, (2) independent certification of process, and (3) traditional product inspection. As the effectiveness of items 1 and 2 is proven, the reliance on item 3 will be reduced. The QIA program will develop a quality architecture, strategies, and plans that should provide for a logical progression from the current product inspection and test methods toward a quality assurance system based on in-process verification methods, which maximize existing sensor technology and deterministic metrology methods. The QIA architecture will be covered in Sec. 2. It and the machine tool characterization are the primary considerations of this report.

The PFMS side of the project emphasizes a quality architecture that allows the retrieval of sufficient amounts of data for determining whether the process is within limits. If a manufacturing process began to produce a poorer quality product, the assessment of data would answer the question of what went wrong and why. The architecture also emphasizes the capability to query the quality database to identify the sources of variability in the machining process and the capability to implement corrective routines for the next batch of parts in order to reduce the process variability to an acceptable level for producing high quality parts.

The AMRF/Navy side of the project emphasizes real-time sensing of process variables and control of the machine tool and the machining process, based on deterministic metrology. The corrections for the variability in the machining process may be applied in the form of: software corrections to the segmented NC code module, (2) real-time hardware corrections, which modify the responses from the machine's positional feedback, and (3) changes to manipulative variables such as feed, speed, and depth of cut. The choice between hardware error correction and software error correction will depend on the time-variant characteristics of the errors and the facility for making the error correction. A possible example for using the third option is to sense the ultrasonic pulse that is generated by surface roughness and to manipulate the feed to reduce surface roughness. This topic is discussed in Sec. 7 , and the second option is discussed in Sec. 5. 
The QIA approach includes development of in-depth understanding of the baseline capabilities of the machine and the phenomena that cause variability in the machining process, development of sensors that can measure process parameters that cause variability, and application of control strategies that reduce the variability in the machining process. Baseline capabilities of machines will be obtained from machine tool characterization, which quantifies geometrical positioning errors of the machine. Error fields will be stored within the quality controller for later use by the quality controller, either in the real-time correction mode or in the software correction mode. This technique of measuring the machine errors and using the error map to correct for machine errors is sometimes referred to as pre-calibrated error compensation [1]. The technique can be used to calibrate the machine for a variety of workpieces. QIA uses this technique to calibrate the machine for two unique test pieces, the NAS 979 test part for milling and the BAS test part for turning. The machine tools and the test parts will be covered in Secs. 3 and 4 , respectively.

This QIA strategy also makes extensive use of process intermittent gauging techniques, which in turn will require a new hardware development that can provide a high speed on-machine probing capability. This hardware is currently being developed and will hereinafter be referred to as the Real-time Error Corrector (RTEC).

Ideally, the inspection results of a part inspected on the machine tool would be identical to the inspection results from a CMM. In reality, we expect differences. Therefore, verification of the processintermittent inspection results, using end-product attributes obtained from CMM, will be performed. Our goal is to maintain a tolerance better than \pm 0.001 inch $(25 \mu \mathrm{m})$ for every dimension specified on our test pieces. Correlation of the two sets of inspection results to identify any offsets in dimensional patterns are essential to the process verification. The initial inspection results are covered in Sec. 4. 
This QIA experiment is being carried out on an array of equipment consisting of the following:

1) A VMC-75 Monarch Vertical Machining Center, with a GE-2000 CNC Controller.

2) A White Sundstrand Horizontal Machining Center, with a Microswinc CNC controller.

3) A Monarch Metalist Turning Center, with GE-2000 CNC Controller.

4) The AMRF inspection workstation, consisting of a Cordax coordinate measuring machine, with an HP 9920 controller.

Each machining center has a quality controller which is interfaced to the machining center's CNC controller. This system consists of a Zenith PC-AT compatible computer equipped with a IEEE-488 communications board linked to an HP data acquisition system that controls an array of sensors. Each quality controller is linked to the machine tool's controlier via an RS-232 interface. All of these quality controllers will communicate with the QIA database and the inspection work station via the communications network.

An important requirement of the QIA project is to make quality information readily assessible to all points of interest via a quality monitor. A wealth of information in numeric form will not suffice because it is too burdensome to analyze. The QIA quality monitor will rely heavily on graphical representation of quality information; thus giving the operator the capability to visualize the many aspects of the inspection results. Small differences between part measurements and the ideal part will be exaggerated. The development of reliable and user friendly interfaces between man and machine is an important goal for the QIA monitor. Initial work will be discussed in Sec. 8 . 


\section{AN ARCHITECTURE FOR QUALITY CONTROL IN THE QIA PROJECT}

T.V. Vorburger and J.C. Boudreaux

\subsection{ARCHITECTURE OVERVIEW}

The Quality in Automation Project aims to develop a quality assurance system that demonstrates deterministic metrology [2] in an automated environment, specifically with the machine tools of the Automated Manufacturing Research Facility (AMRF). Deterministic metrology is the proposition that under certain conditions a manufacturing process and its environment can be monitored and controlled so as to avoid completely the production of bad products. The conditions are: (1) an automated environment so that human frailty is not a factor, (2) sufficiently uniform or well-characterized materials, and (3) a manufacturing process sufficiently well understood that its process variables can be directly linked to their effects on quality. The QIA system will combine quality assurance methods, such as post-process inspection, process-intermittent gauging, real-time sensing, and statistical process control with the tools of automation such as process planning, network communications, and automated numerical code (NC) generation. We further intend that the QIA system architecture be generally applicable to other automated manufacturing systems, such as the Precision Flexible Manufacturing System (PFMS) demonstration project of the Department of Energy.

When the QIA system is in place, the operation of the AMRF will be enhanced in several ways. The following scenario describes key steps in the manufacture of a part on the AMRF using the quality assurance capabilities to be developed by the QIA project.

\section{QIA Scenario}

1) A part design is developed on a CAD terminal tied to the quality network. The system provides appropriate responses concerning whether or not the requested tolerances are achievable with the available manufacturing equipment. 
2) A process plan is developed that includes archival information about the high-level manufacturing operations to be performed. The numerical code for the machining operations is also generated in a standard format (EIA RS-274D). In the AMRF, this is done independently of the process plan development. At this stage the machining numerical code is in its archival form. It is neither specific to a particular machine tool controller, nor does it include any capability for tool path correction.

3) The archival code is then reformatted into segmented code that contains NC chunks equivalent to at least one pass of the tool through the workpiece. The segmented code also contains language conventions. that are specific to a particular machine tool. The segmented code is stored in the quality controller (QC) for each machine and the chunks are then sent, one at a time, to the machine tool controller for each sequence in the cutting operation.

4) The QC houses a program that can change the operation of the machine in several ways based on sensor data. For example:

- Detection of temperature changes on the machine can lead to a correction of the tool position based on a model of the machine distortions as a function of temperature.

- Detection of excessive vibration or force on the tool can lead to a machine shutdown, a change in the machining conditions, or a change of tool.

- Detection of excessive surface roughness can lead to a machine shutdown or a reduction in the feed.

5) The tool path corrections are generated either from real-time measurements of the thermal state of the machine or from processintermittent data gathered during a part probing cycle as discussed below. The resulting correction steps are implemented by either a software or hardware approach. In the software approach, the correction steps are integrated into the segmented chunks of NC code housed in the QC. This adapted code is then sent to the machine tool 
controller. In the hardware approach, the correction steps are sent to a special processor called the real-time error corrector. This unit intercepts the pulses traveling from the machine tool encoders to the machine tool controller and changes them to accomplish the desired path correction without changing the NC code residing in the machine tool controller itself.

6) Between sets of processing steps, the QC monitors part accuracy by comparing touch-probe measurements with expectations based on the design. The QC can provide additional corrections to the tool positions. This is accomplished by modifying the segmented code blocks based on the touch-probe data. The QC can also provide information for someone to change the correction factors for the next part. The probing scenario is generated either automatically or interactively from the design data.

7) After machining, the part is measured in an inspection workstation. The part geometry is compared to the original design and to the results of the measurements on the machine. If significant errors occur, another set of correction factors is generated for the machine. These are stored in the QC. For this step, it would be desirable if the inspection station were at least a factor of three more accurate than the machines.

8) The quality data from the machining and inspection processes are integrated into overall quality evaluation statistics and displayed on the quality monitor. These displays include control charts, scatter plots, and correlation charts that monitor functional dimensions of the parts and process variables as functions of time. Two types of artifacts are used as control checks in the system. One is a previously manufactured artifact that can be probed on all of the machines and on the IWS. The other is a standard package containing the design, process plan, and NC program for a test workpiece that can be machined on an ad hoc basis. 
The completion of a prototype QIA system will involve a number of hardware and software tasks including:

- Development of a system architecture,

- Interferometric characterization of the machine tool errors as a function of machine thermal cycling,

- Development of models for tool path correction,

- Development of software code for tool path correction,

- Installation of the network communications,

- Development of the real-time error corrector hardware,

- Development of the programming environment,

- Development of post-process inspection scenarios and software,

- Development of process-intermittent gauging hardware,

- Development of process-intermittent gauging scenarios and software,

- Development of real-time accelerometer sensor hardware and software,

- Development of a force monitoring system for sensing tool wear,

- Development of sensors for surface roughness measurement,

- NC coding for the machining and inspection of the test parts,

- Integration of the subsystems into the main quality monitoring system.

An overview of the project milestones is shown in Figure 2.1 with projected starting dates and task durations (in days).

In the following subsections, we describe the proposed system architecture as of September 30, 1988. Section 2.2 describes the control loops that form the heart of the system. Section 2.3 contains the data requirements for the system. Section 2.4 discusses the programming environment and Sec. 2.5 briefly discusses the QIA network and depicts how it will interface with the AMRF. We envision that this architecture will be modified as we carry out the hardware and software tasks leading to completion of the prototype quality monitoring system.

\subsection{CONTROL ARCHITECTURE}

The system architecture for quality in automation depends on four control loops. In each loop, certain characteristics of the workpiece or the machining process are measured, and corrective actions are taken if 


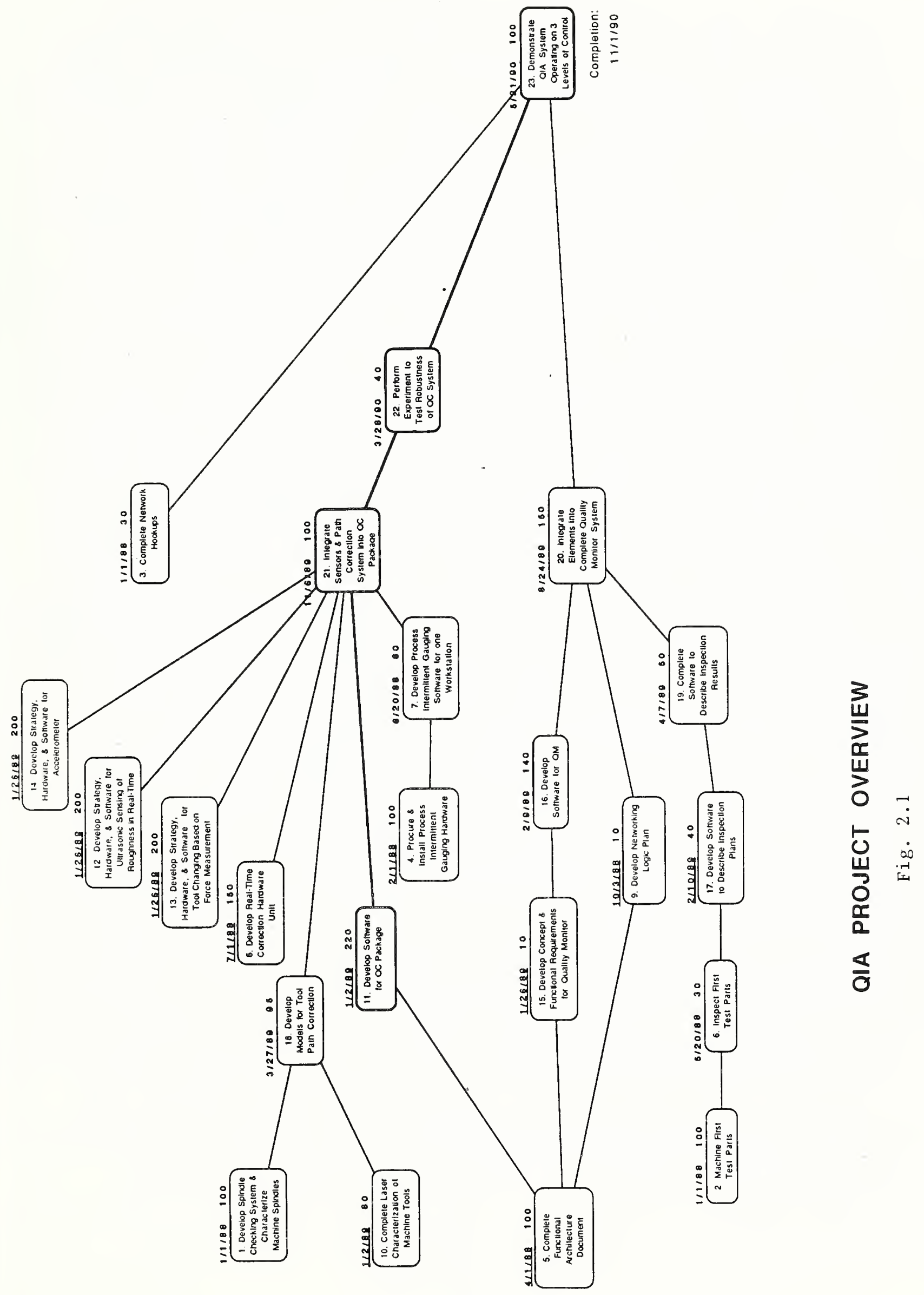


necessary. These control loops are called pre-process characterization, real-time sensing, process-intermittent gauging, and post-process gauging. The character of each level of control depends substantially on the type of measurement being done.

In this section we discuss the types of sensing that can be done and the control strategies that can be used with each. We further identify those control strategieș that may be readily adapted now into the QIA project and try to identify fruitful research areas in quality control of automated machining for the project.

In general, we are guided by the observation that the quality of a machined part depends on the accuracy of its dimensions, the accuracy of its form, and the amplitude of its surface texture. At this point, surface integrity, which includes the concepts of defects and residual stress, is considered to be outside the scope of the project.

Dimension and form are affected by such factors as path errors during machining, tooling size errors, and thermal changes in the workpiece. The surface texture includes both roughness and waviness [3] and also depends on several factors, including machining vibrations and machining conditions. The machining conditions that should be closely controlled are the machine feed and tool quality, but machining speed and possibly depth of cut also affect surface texture. In addition, the machinability properties of the workpiece material itself control the resulting surface texture. Knowledge of machinability properties can be useful for realtime or process-intermittent control, but this aspect of control is outside the scope of the QIA project. In QIA the major areas of concern for quality in machining are the sources of dimension and form errors, unacceptable vibrations, and tool quality.

\subsubsection{PRE-PROCESS CHARAGTERIZATION}

The pre-processing loop (Fig. 2.2) consists of two major activities:

(1) characterizing the thermal stability of the machine tool and 


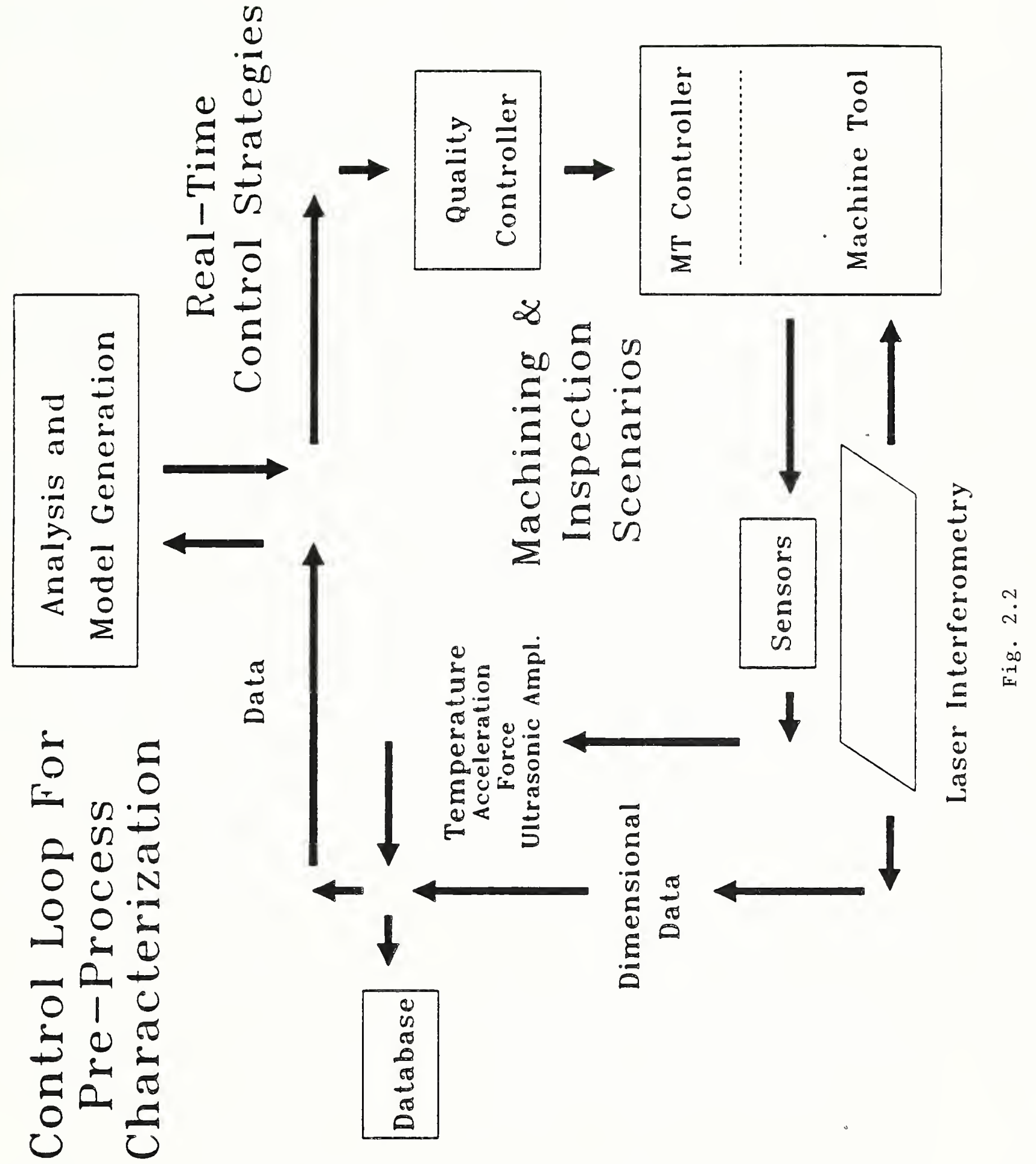


(2) taking laser measurements to determine the geometric accuracy of the machine tools as temperature varies. We installed forty thermocouples on each machine to determine those temperature sensitive locations that contribute to thermally induced deformations of the machine that would cause unwanted deviations between the tool and workpiece. The positions for each are discussed in Sec. 3.

From the combination of laser interferometry and thermal data, we are developing the models of the machining path errors as functions of position and temperature. These models will be used in the other three control loops. Therefore, in the pre-process loop, the sensed quantities are the interferometric and temperature data, and the outputs are the geometrical and thermal models of the machine path errors and the set of control strategies to be used in the other three loops. We will implement these control strategies at the beginning of the project and modify them only occasionally after that, whereas the other loops will be running on a daily basis.

The architecture of the pre-process loop should also allow for the generation of other control strategies besides dimensional corrections. Therefore, experiments that yield information leading to the development of such control strategies are also part of the pre-process control loop. The sensed quantities include vibration or force on the machine or the roughness of the workpiece. These will be discussed further in Sec. 2.2 .2 .

\subsubsection{REAL-TIME SENSING}

Real-time sensing refers to those measurements that are made while the part is being machined. Quantities that may be sensed at this stage are the thermal state of the machine, the vibration level, cutting forces, and possibly surface roughness. We describe below a method for sensing each of these quantities and how the results may be used for real-time control of the machining process. A conceptual diagram is shown in Fig. 2.3. 


\section{Control Loop for \\ Real Time Sensing}

Signals to Operator

Quality

Controller

\section{Program Segments:}

Corrected NC Code

Temperature or

Correction Pulses

rms Acc. Machine Shutdown

Force Machine Shutdown

Ultrasonic Ampl.

Feed Reduction

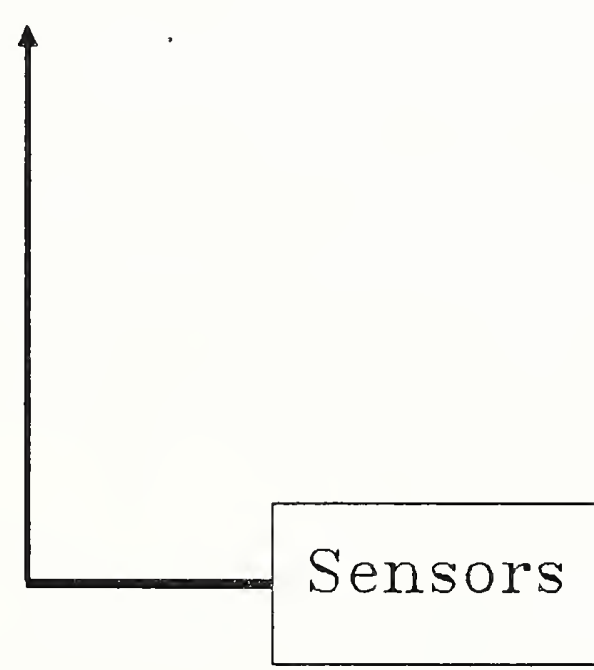
or

Machine Shutdown

Requests

MT

Controller

Machine Tool

Fig. 2.3 


\section{Thermal State of the Machine}

Thermal sensing is performed with an array of thermocouples to be discussed in Sec. 3. The thermal conditions that result in deforming the structural loop of the machine tool will become inputs to the empirical model. We are designing this model to analyze the thermal data and to calculate the tool path errors as a function of temperature.

The procedures we are using are based on techniques previously developed at NIST [4-6]. The model of machine thermal distortion is being developed for each machine by the laser interferometry measurements of the machine distortions discussed in Sec. 2.2.1. The resulting model must be simple enough so that the tool path correction can be calculated by the in-line quality controller in real-time ( $0.1 \mathrm{~s})$.

The corrections will be accomplished in one of two ways depending on the speed required. First, we are developing methods to segment the NC machining code into chunks before we send it to the machine tool controller. The quality controller will correct these chunks of code. The correction may involve changing only one or two parameters in the NC code such as the endpoints of a straight tool path, but it more likely will involve explicit correction of each interpolation step in a tool path to produce accurate form in the part (see Sec. 2.4.1).

For higher speed requirements, the positional feedback signal that the machine tool controller normally receives can be "corrected" by appropriate interjection of pulse signals that simulate measurement data from the machine scales. NIST is developing the hardware for implementing this approach and this device is discussed in Sec. 5. The software driver is described in Sec. 2.4.2.

\section{Vibration}

The vibration level directly affects the waviness of a machined component. Vibration may be sensed by an accelerometer located on the tool post of a lathe or on the part fixture of a milling machine. The 
vibration measurements will likely be of a simple nature. That is, measurements may be taken of the rms acceleration level integrated over the audio bandwidth $(\sim 20 \mathrm{~Hz}-15 \mathrm{KHz})$ at a sampling rate of $10 \mathrm{~Hz}$. It may also be beneficial to measure the spectrum of the vibration signal, but that procedure is not included in the present plan.

In the initial stages of the project we will invoke a simple strategy to deal with the accelerometer signals. If the rms acceleration exceeds a certain level, we will back the tool off from the workpiece, stop the machine and signal for manual intervention to correct the problem. To implement this "Stop" and "Backoff "strategy, we will activate the electrical closure of the "Feed Hold" and "Clear" buttons on the machine toll controller. Then we will download instructions to stop and back off. The threshold acceleration level for directing a shutdown will depend on the machining operation and the part size, shape, and material. That level must be learned by experimentation for each process.

A more elaborate adaptive control strategy can also be devised. For example, in a rough cutting procedure, the cutting speed could be changed by a significant factor in order to change the driving frequency of the vibration. Or a search algorithm through feed and speed conditions could be devised to reduce the measured rms acceleration to an acceptable level. Alternatively, a table of recommended cutting conditions for various materials and processes could be installed in the database. Such a table might be derived from available machining data [7] or from knowledge gained during the QIA project.

However, the last approach is really a setup strategy rather than a control strategy. In any case, the more elaborate procedures constitute the subject of a possible research investigation in the QIA project. Our first implementation will be the simple shutdown strategy discussed first.

\section{Force}

The condition of the tool affects both the quality of the parts and the productivity of an unmanned, automated machining operation. The QIA 
Architecture will enhance quality and productivity by monitoring tool wear. We have considered the following two approaches to monitoring tool wear: (1) using process-intermittent gauging to inspect tool geometry for wear, (2) using real-time force sensors to monitor unacceptable cutting forces.

The latter option would offer continuous monitoring of the tool's cutting force and the likely detection of unacceptable tools. Rapid changes in cutting force should indicate an unacceptable, worn tool and likely degradation of the texture of the machined surface. Figure 2.4 shows the tool force versus time relationship for a hypothetical turning process. As the tool becomes worn, the wear land on the cutting edge increases, leading in turn to increased cutting force. This observation suggests that we can use the tool force as an indicator of tool wear. However, we need to learn from research the force thresholds that warrant a tool change for: (1) various tools, (2) various cutting operations, and (3) various materials.

Better sensors for measuring tool force are also needed for this approach. Commercial sensors for measuring the tool force in turning applications have been developed elsewhere $[8,9]$. If some of the above limitations are solved, then tool force sensing could offer a more functional gauge of tool performance than tool geometry inspection. Therefore, the first implementation of the QIA architecture will use cutting force sensing as a simple indicator of tool wear and as a signal to the quality controller to change the tool. This work will rely to a large extent on sensors and strategies developed in other laboratories.

The monitoring of tool geometry by using process-intermittent gauging is discussed in Sec. 2.2.3.

\section{Ultrasonics}

We are designing the QIA architecture to include surface monitoring and control with an ultrasonic sensor. The architectural structure will provide two modes for monitoring surface texture with ultrasonics. The 
Tool Force

as an

Indicator of Wear

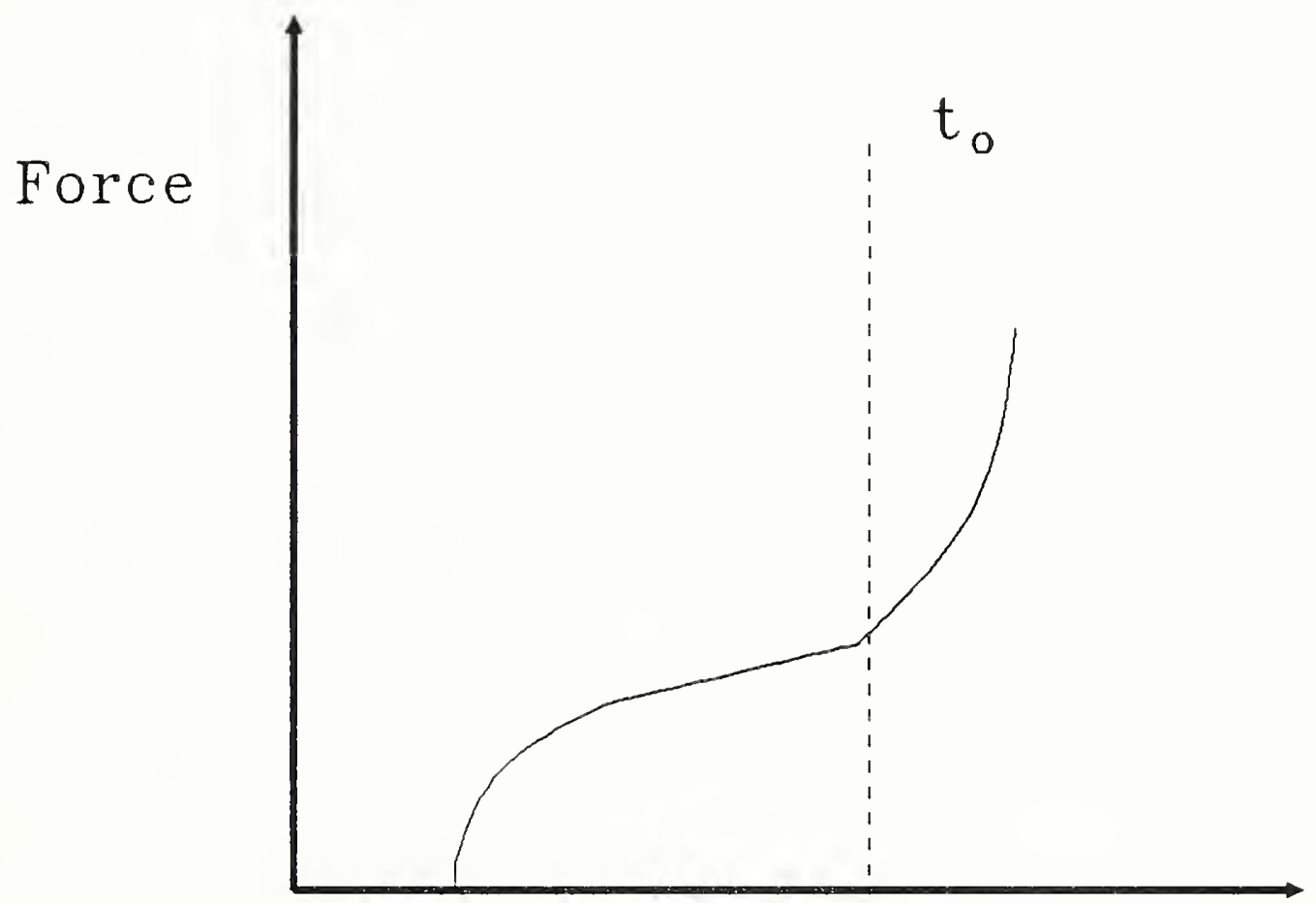

Time

Fig. 2.4 
first is as a real-time sensor for surface roughness. This monitoring system is based on the principle that the reflected amplitude of an ultrasonic pulse is generally inversely related to the surface roughness [10]. Second, we might also use this technique as a process-intermittent surface roughness gauge between machining steps as discussed later. The information from the gauge will be complementary to that from the vibration sensor, because vibration in the process is a direct cause of waviness.

In addition to monitoring surface roughness, the architectural structure can include a facility to control surface roughness. For example, if the sensor gives an excessive roughness value, the control response might be to decrease the tool feed by one third. The usefulness of such a strategy would depend on the tool and the workpiece material. It is probably an acceptable strategy for high-speed steel cutters used in milling certain types of workpieces. If after changing the initial conditions, the sensor still produced an excessive roughness value, the machine would be shut down and the quality controller would call for manual intervention by the operator.

The other monitoring capability that ultrasonics may provide is to measure the delay-time in the reflected ultrasonics pulse. This delaytime can serve as a basis for measuring critical dimensions such as the wall thickness of the workpiece. The ability to make this measurement will depend on the results from another research project (outside of the scope of the QIA project) that will test the practical use of this approach.

The above considerations mainly apply to cylindrical and prismatic work pieces. More research must be done before we can effectively perform real-time monitoring of surface roughness on a hemispherical component. The nosepiece of the modified BAS turning part that we will machine in the QIA project is a case in point. Because the normal to the surface changes during machining of a hemisphere, it is difficult to hold a gauging system normal to the surface as the machining proceeds. On the other hand, an oblique scattering geometry would likely be much more difficult to 
implement for real-time sensing of surface texture, since it would likely require careful alignment between a separated source and receiver. A scanning mechanism for producing normal incidence on spherical parts is under development under a separate NIST project in cooperation with DOE.

\subsubsection{PROCESS-INTERMITTENT GAUGING}

The term process-intermittent gauging refers to measurements performed on the workpiece or the cutting tool while they are fixtured on the machine but machining is not taking place. This primarily means measurements taken during setup or after machining, just before releasing the part from the fixture.

We are designing the QIA architecture to employ process-intermittent gauging to acquire data rapidly on the following manufacturing parameters: part dimensions and form, part orientation, tool setting, surface texture. of these, part dimensional measurements using on-machine probing and surface roughness gauging are receiving most of the current effort.

Figure 2.5 shows the conceptual design for the control loop. The numbers in this figure indicate a chronological order: measurement commands followed by results followed by modified process commands from the quality controller. We discuss below the four types of measurement operations in process-intermittent gauging.

\section{Part Dimensions and Form}

We are constructing prototype hardware to implement the conceptual design of Fig. 2.5 (see Sec. 5). The high-speed dimensional measurement of machined parts using on-machine probing will be accomplished with the RTEC, which will continually update the position coordinates of the machine tool so that this information can be read rapidly by the quality controller (with access times on the order of $1 \mu \mathrm{s}$ ). Such a procedure will overcome the access-time limitations associated with reading the position coordinates from the machine tool controller itself [11]. From a mechanical point of view, the probing movements can take place at high 
Control Loop for

Process-Intermittent Gauging

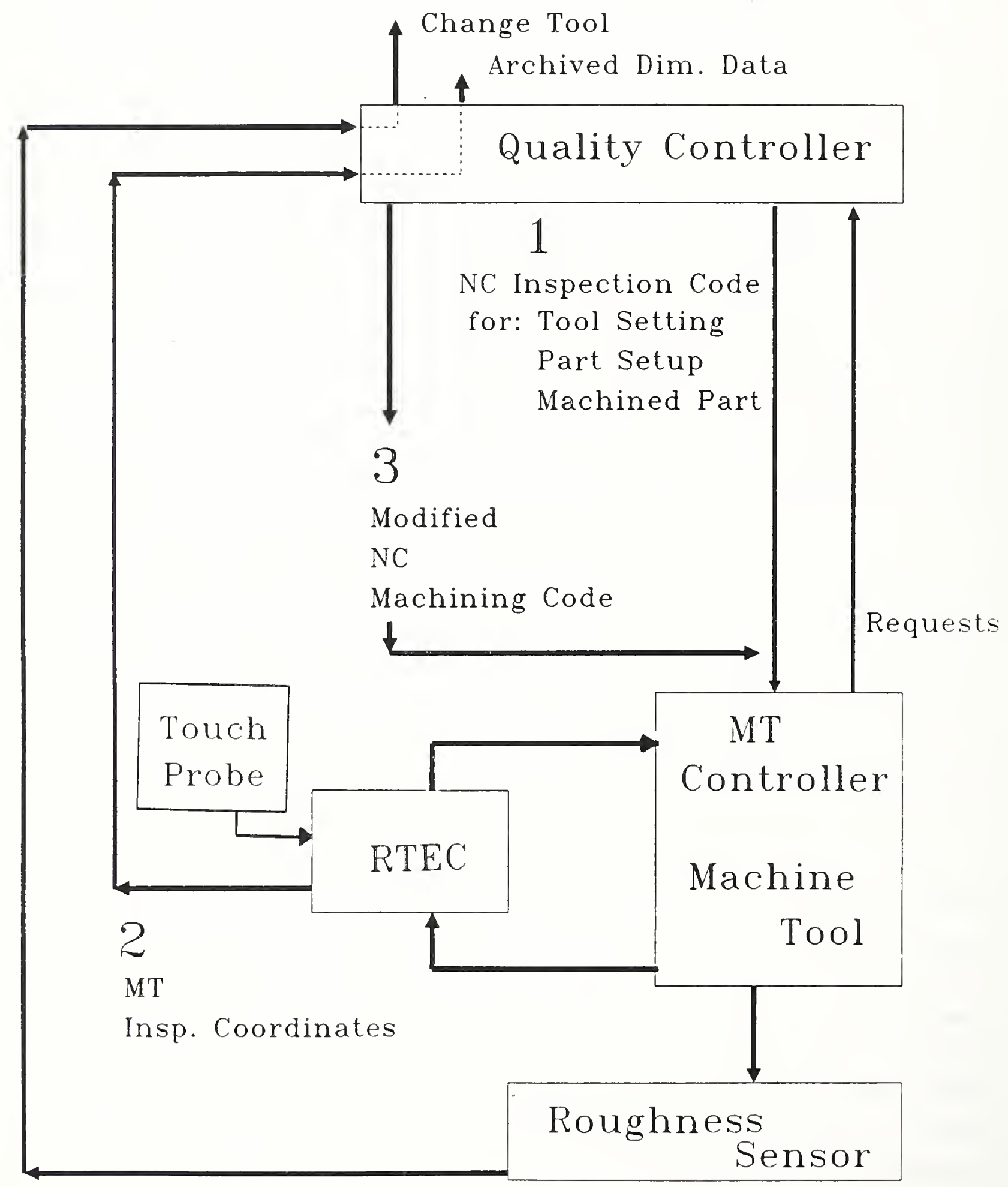

Fig. 2.5 
speed ( $200 \mathrm{in} / \mathrm{min}$ ) because the part dimensions and orientation are well known, owing to the fact that the part has just been machined to a specified form and is still located in the machining fixture.

We have developed options and strategies for using the on-machine probing data. The basic strategy will involve a roughing cut, and possibly a semifinish cut, followed by a series of rapid probing measurements of the part, followed by the finishing cut. We will design the quality controller software to use the probing information between cuts to make a path correction in the finishing cut if necessary.

For certain types of machining errors, a direct path correction can be performed. That is, the measured form of the part can be compared to the expected form, and the subsequent correction to the tool path in the finishing cut will simply be the geometrical difference between the two. Such a procedure is feasible for certain types of path errors including springback errors, dimensional changes in the tool due to wear, and firstorder spindle growth. However if, for example, the springback error is different in the finishing cut than it is in the roughing cut, a direct path correction, based on form errors measured after the roughing cut, will lead to error of form in the finished part.

This scenario is only one of several types of machining conditions for which direct path corrections will lead to trouble. However, that observation suggests that an iterative machining procedure involving a roughing cut followed by several cycles of dimensional probings and dimensional cuts, followed by a finishing cut, might be an effective approach to accomplish high dimensional quality in automated machining. Although such an iterative procedure would require more machining time, it could be more general and more straightforward to develop in comparison with a menu of procedures that depend on cutting conditions.

It should be noted that the problem of part distortion due to stress relief after machining is one that we will deal with by a combination of post-process and process-intermittent gauging. A second question has to do with the probing algorithms that are required for effective part 
probing. In particular, how many data points are needed for adequate measurement of tool path error in every situation?

In any case, the application of process-intermittent part probing is certainly a subject for research in the QIA project.

\section{Part Orientation}

It is crucial to measure the orientation of newly fixtured parts during setup to make sure that they are properly positioned in the machine fixture. This procedure is important for unmachined blanks as well as for machined parts that are being refixtured. In either case the measurement scenario is a series of NC touch-probing steps quite similar to that described under "Part Dimension and Form". The measurement results can either lead to a path correction if the orientation errors are small or to a signal that manual intervention is required and the part must be refixtured.

\section{Tool Setting}

Automated tool setting stations will be installed in each of the three machines in the QIA project. The tool setting stations will measure the length, position, and radius of the tool for milling machines and the tool tip position for turning machines. The equipment for accomplishing both the tool setting operation and the part probing operations discussed before will be made available to the project through an agreement with Renishaw Corporation.

\section{Surface Texture}

It is also important to inspect the surface texture of the part after machining steps and particularly after the finishing cut is made.

Excessive roughness often indicates that the tool has become worn, whereas excessive waviness usually indicates that excessive vibration has occurred during machining. Either condition warrants manual intervention to correct the problem. 
We are considering the following options for process-intermittent measurement of surface texture. For the first implementation of the QIA system, we will use the ultrasonic roughness sensor as a real-time device for process-intermittent inspection on the turning machine. Until now this technique has been limited to fairly rough surfaces (roughness average $>1 \mu \mathrm{m})$ [10] and long surface wavelengths $(\lambda>150 \mu \mathrm{m}$ for an ultrasonic frequency of $10 \mathrm{MHz}$ ). It should be noted that the probing wavelength is approximately equal to the lower limit of detectable surface wavelengths, depending on the angle of incidence of the radiation with respect to the surface. In principle the ultrasonic gauge may be easily operated at frequencies as high as $100 \mathrm{MHz}(\lambda-15 \mu \mathrm{m})$. Results on its sensitivity to surface roughness at this frequency are not yet available.

An alternative option is the commercial infrared sensor [12, 13] currently used on the inspection workstation in the AMRF. Because the probing wavelength of this one is $0.8 \mu \mathrm{m}$, this technique is complementary to the ultrasonic technique. Its range of measurable $R_{a}$ values [3] for fine machined surfaces is $-0.5 \mu \mathrm{m}$, almost equal to the resolution of the ultrasonic technique, and its range of measurable surface wavelengths $(-0.8 \mu \mathrm{m}-30 \mu \mathrm{m})$ is much shorter than that of the $10 \mathrm{MHz}$ ultrasonic sensor.

The foregoing analysis of options suggest three research topics for study in the QIA project.

First, the upper sensitivity limit of $0.5 \mu \mathrm{m}$ for the infrared gauge has only been identified for surfaces with fine machining marks produced by hand lapping. Its range should also be tested for machining processes like milling, facing, and turning that leave widely spaced feed marks on the surface.

Second, both the infrared and ultrasonic gauges are scattering instruments. A scattering technique with an intermediate probe wavelength between those two should be developed. Possibilities include a $\mathrm{CO}_{2}$ laser system with a wavelength of $10.6 \mu \mathrm{m}$ or an ultrasonic gauge with frequency 
of $100 \mathrm{MHz}$ as discussed above. Either instrument would test a range of surface roughnesses that bridges the gap between the ranges of the other two instruments.

One alternative to the scattering approaches discussed above is the use of a compact stylus-type profiling instrument that can be placed on the part or held against it by a robot. Existing battery-powered, portable roughness gauges could be fitted with transmitters for communicating with a suitable interface to the quality controller. Such a development would make for entirely wireless operation of stylus type, profiling devices in automated machining.

One of the above approaches will be a research topic in the QIA project, but during the first implementation of QIA, the ultrasonic technique will be used for process-intermittent gauging. Other possible alternatives for process-intermittent, roughness gauging include capacitance probes, both profiling [14] and area-averaging [15], optical profiling $[16,17]$ and ultrasonic profiling [10].

\subsubsection{POST-PROCESS GAUGING}

A goal of the QIA project is to provide for a logical progression from traditional product inspection toward quality assurance based on inprocess verification methods. Post process inspection will provide a metrological basis at the start. We will verify the process-intermittent gauging by performing post-process gauging of finished parts. This gauging will be accomplished with an accurate coordinate measuring machine for the measurement of part dimension and form and a stylus type gauge for the measurement of surface texture. The accuracy of the CMM should be significantly better than the required accuracy of the manufactured components. Along the same line, the stylus-type surface texture gauge will provide an accurate, standardized approach [3] to the measurement of roughness and of waviness as well. 
The post-process gauging system will serve as a metrological anchor for the real-time sensing and process-intermittent gauging procedures. The associated control loop is shown schematically in Fig. 2.6.

Post-process gauging serves two main functions: certification and process monitoring. Both functions involve testing of the part against the design tolerances. The data gathered for certification are attributes of the part itself whereas the process monitoring information is associated with the machining systems and with previous parts manufactured on them.

Important considerations for certification are the design tolerances, the measurement algorithms, and part sampling. The design tolerances must be carefully defined so that the part meets its functional purpose without excessive manufacturing expense. Measurement algorithms must then be developed that adequately test those functional tolerances. This consideration is particularly important during the present era in machining technology when functional gauges are being replaced with digital measurement procedures embedded in CMM controls. The number of data points that must be taken for adequate testing against each tolerance is a crucial question here [18].

Part sampling refers to the question of whether to measure every part that is produced in a batch. In some cases and on certain parts, certification can be achieved instead by sampling only a fraction of the manufactured parts provided this is supplemented with process-intermittent and real-time monitoring of both the parts and the process.

The dual function of process monitoring consists, first of monitoring for control and, second, process diagnosis when the measured quantities appear to be slipping out of control. Both functions require the integration of the inspection results into overall quality evaluation statistics including control charts, scatter plots, and correlation charts that monitor key functional dimensions of the parts and key process variables as functions of time. 


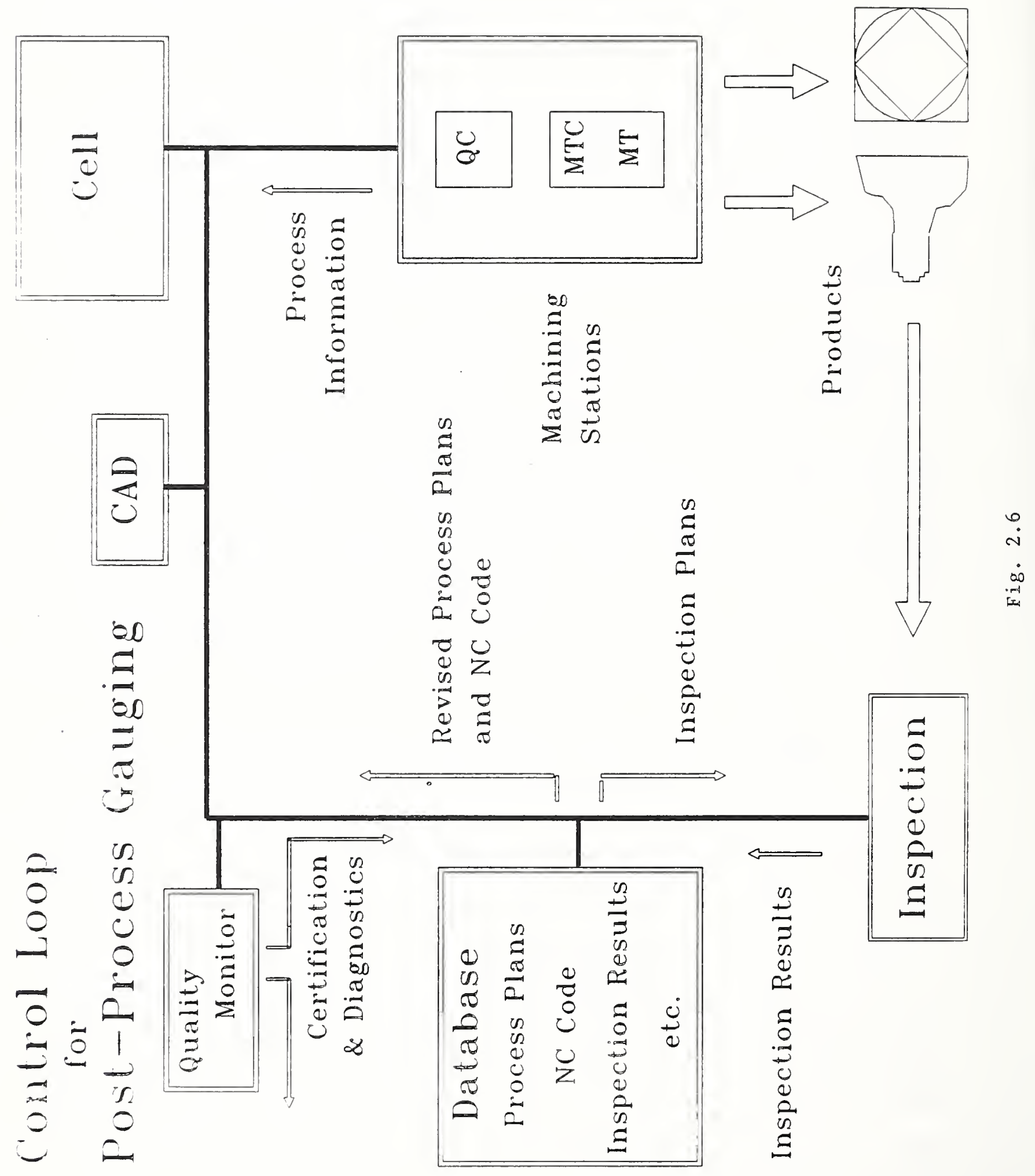


Diagnostic analysis of changes in the quality evaluation statistics will enable us to pinpoint the process errors that caused them and to correct the process. In diagnostic analysis, the graphical display of the component, its tolerances, and the measured deviations in a user-friendly manner will be particularly important features of the quality monitoring system.

For certain types of machining errors, the diagnostic analysis will cause the direct path correction of a machine for subsequent components based on the errors measured on previous components. In other cases, the diagnosis will result in a major restructuring of the steps of a process plan.

During the initial stages of the QIA project, much of the diagnosis will be manual and will involve expert knowledge. However, the continual automation of the quality monitoring function will warrant the development of rules for the inspection measurements, rules for diagnosis and process characterization, and rules for modifying the process plans. An open architectural structure will permit the gradual codification of the rules into automated quality subsystems that can coexist with human manipulation, oversight, and intervention.

\subsubsection{GENERAL}

The entire control loop structure is shown in Fig. 2.7. It suggests that in order to simplify the architecture, there should be a bifurcation of duties between the quality controller (QC) and the system comprising the database and the quality monitor (QM).

The quality controller will be working closely with the machine tool controller. It will receive the sensor data during real time sensing and process intermittent gauging operations and send back the appropriate correction responses or stop commands to the MTC. The QC will also condense and reformat the sensor data for archiving by the quality monitor. The database, on the other hand, will receive the data generated 


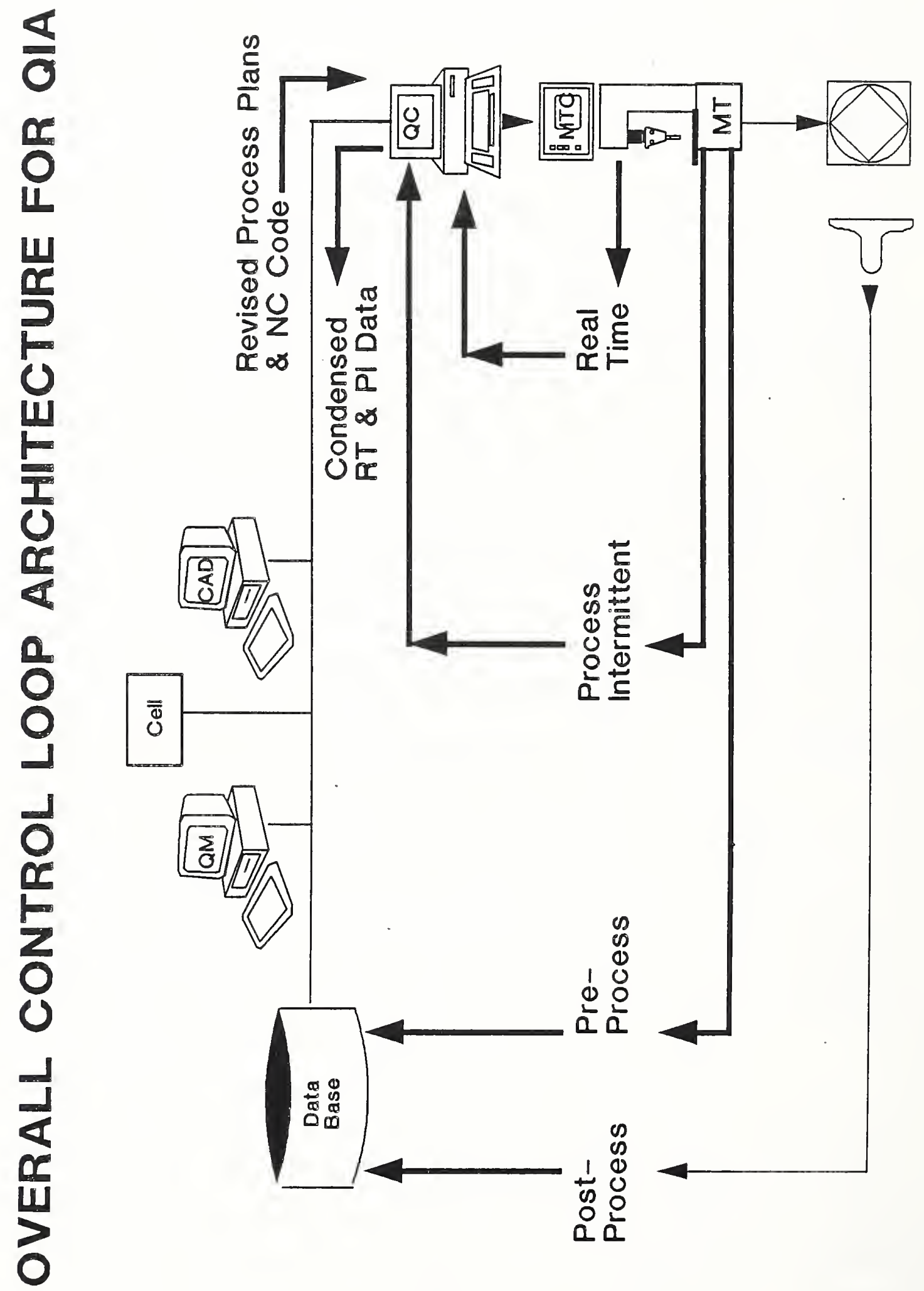

i 
in the pre-processing and post-processing operations. The stored data will then be directly available to the quality monitor for analysis.

The potentially large volume of real-time data will likely have to be condensed in the QC before being archived in the database. On the other hand, the amount of process-intermittent data is not likely to be large; therefore, it can be transmitted through the QC to the database without having to be condensed.

\subsection{DATA REQUIREMENTS FOR AUTOMATED MANUFACTURING}

\subsubsection{OVERVIEW OF DATA REQUIREMENTS}

This section reflects points of mutual agreement reached in discussions concerning the design of the associated data management system. Data requirements in the QIA and PFMS projects are expected to be similar. Because of the complexity of the issues involved, this section is intended to be used as a basis for subsequent technical discussions leading to more precise specifications for data requirements in flexible automated machining systems. The data management system has five components: the CAD cluster, the process planning cluster, the partprogramming cluster, the real-time support cluster, and the certification cluster.

\section{- 2.3.2 THE CAD CLUSTER}

The QIA data management system will need access to archival data elements generated during the part design process. The information usually available from CAD systems includes part geometry and topology, dimensioning and tolerance data, material type, part features, surface finish, and other categories of engineering data elements.

Part geometry and topology data provide the information necessary to represent the geometry of the part in a computer-readable form and the interconnection of geometric design features. Part geometry may be represented using wireframe information structures. More complicated 
geometric features are described by splicing together simpler features or by introducing spline functions, i.e., piecewise continuous polynominal interpolation functions. More advanced geometric representations include boundary representations and constructive solid geometry.

Dimension data give information about the magnitude, or size, of specific features of the part so that all sizes and shapes can be determined without calculating or assuming the magnitude of any unrepresented dimensions. The following dimensions are represented: dimension, diameter dimension, linear dimension, ordinate dimension, point dimension, and radius dimension. Tolerancing data must also be included. These data elements express allowable errors on dimensions. In addition to conventional tolerancing, other methods may be used in part design specifications. One frequently cited example is geometric tolerancing, which specifies tolerances of a part's form features, including: straightness, flatness, roundness, cylindricity, parallelism, perpendicularity, angularity, concentricity, and symmetry.

The definition of data elements in the cluster is being done at NIST as part of the Product Data Exchange Specification (PDES) effort. This standard is being developed by the active collaboration of both U.S. national and international groups. Because this effort has widespread support throughout the Department of Defense and also in the private sector, the most rational course of action for the QIA project is to adopt this approach as the defining basis of this data cluster.

Reference [19] contains the current draft of the physical file format which will be used to represent PDES data elements.

\subsubsection{THE PROCESS PLANNING CLUSTER}

The QIA data management system will include process plans that describe steps in the manufacturing process above the level of the NC machining code. 
The process plan contains a description of operations to be performed at each workstation, including material handling operations, fixturing operations, tooling operations, machining operations, transfer instructions for parts, and protocols for both process-intermittent and end-process inspection.

Within AMRF, process planning is understood to be keyed to part features, which implies that there must be a complicated linkage between this cluster and the CAD cluster. Since a flat file format for process planning has already been developed within the AMRF, we propose that QIA use this format as a basis for subsequent development. A technical overview of the AMRF flat-file format is found elsewhere [20].

\subsubsection{THE PART PROGRAMMING CLUSTER}

The QIA data management system will store a nominal part program for each part. Part programs will conform to the EIA RS-274D standard [21]. However, no agreement has yet been reached about the conditions which a part program has to satisfy in order to be accepted as nominal. For example, a standard-conforming part program may contain a line which commands that the tool trajectory be circular (preparatory functions G02, G03). Though it may seem reasonable to allow this in a nominal part program, the effect of this would be to introduce another source of nonrepeatability by relying on system-specific features of NC machines to resolve the linear interpolation method. Therefore, it is probably a better idea to restrict nominal part programs to those in which the tool trajectory is explicitly linear.

Since one of the major thrusts of this project is to improve the quality of manufactured parts using the techniques of adaptive machining, it is essential that careful consideration be given to what we call the segmentation problem. Given a fairly complicated engineering part, the normal conventions are to develop several part programs and then to decide the specific order in which to run them. This means that the nominal part program is really a set of programs, each one of which is a complete part program. Not only must the data management system keep track of these 
segments, which itself requires a great deal of interaction with process planning, but it must also keep track of the specific order in which the components are passed to the NC machine controller for execution. Since the order of execution is a data element of each step in the procedure section of a process plan, this requirement is easily satisfied if the part program is segmented in the same way as the process plan. However, it is not yet clear that we can always achieve such a tight coupling. In fact, we might rarely achieve this tight coupling if we decide to compensate for systematic errors by modifying the nominal part program itself, i.e., by adding correction factors to every point in the tool path.

\subsubsection{THE REAL-TIME SUPPORT CLUSTER}

A great number of data elements will be needed to support real-time control and diagnostic analysis of the manufacturing history of the part. These are classified into the real-time support cluster, discussed here, and the certification cluster.

Assuming that a rationally segmented part program has been generated, the reduction of further systematic error requires us to compensate for changes in the machining environment. Such changes may only be detected by the real-time acquisition and processing of sensory data. Once detected, appropriate compensation can be done either by modifying the next program segment, or by sending correction signals to the real-time error corrector (RTEC).

The real-time support cluster consists of the real-time and process intermittent data gathered by the quality controller and will include the following:

Real-time Data:

- Temperature profiles of key thermal sensors as a function of time,

- Acceleration, force, and ultrasonic amplitude profiles and the derived roughness or waviness values, 
- Machining conditions extracted from the process plan.

Process-Intermittent Data:

- Temperature profiles as before,

- Expected profile of the machined component,

- Deviation profile as measured by the touch probe,

- Optical or ultrasonic signals for surface texture and derived roughness or waviness values.

The database in the QC for handling the above information will use a variant of the Standardized Query Language (SQL) [22] presently used in the AMRF database.

The signal profiles from the acceleration, force, and ultrasonic sensors will likely be filtered and condensed in the quality controller before any shutdown actions are initiated.

The measured signals from these sensors will consist of a stream of voltage signals that remain constant or that vary by small amounts if the machining process is in control. These data may be input to the QC at a rate of 1 per second for each measured value. Given these inputs, an appropriate summary criterion for action by the quality controller might be the average value of each sensor's data over a 1 minute interval together with the standard deviation of the readings and the highest and lowest measured values during the 1 minute interval.

The temperature data will be handled somewhat differently. Because the temperatures of the machine tool elements change slowly, the thermocouple array may be sensed at a rate as slow as once per minute. However, all of these data will likely be stored in the quality database during a machining operation because of their critical influence in the path correction strategies adopted by the QC. 


\subsubsection{THE CERTIFICATION CLUSTER}

The purpose of the certification cluster will be to provide a complete record of the manufacturing history of each part and of the characteristics of each machine so that an operator at the quality monitor can detect incipient changes in the machining process or can reconstruct the causes of extraordinary lapses in part quality.

The certification cluster will include the pre-process and postprocess inspection results as well as the condensed data from the realtime cluster. All of these will be archived in the quality database.

At this stage of the project, it appears necessary to archive every data point measured for dimension and form assessment. We also plan to archive surface texture profiles obtained from post-process inspection. The vast quantity of surface texture profiling data may eventually warrant the deletion of such data from the system and the archiving of appropriate roughness and waviness statistics instead, depending on the intended function of the surfaces of the manufactured components.

All of the process monitoring functions described above will be accessible on at least one user-friendly terminal called the quality monitor, which will communicate with the database via the network. This will be a complete monitoring system. Sub-elements of it may be duplicated elsewhere in the AMRF/quality network.

\subsection{PROGRAMMING LANGUAGE ENVIRONMENT}

Since the primary effort of the QIA Project is to identify and reduce sources of systematic error throughout the manufacturing process, the architecture is being designed to support: (1) real-time acquisition and processing of sensory data obtained from thermal, vibration, force, and ultrasonic sensors; and (2) adaptive machining algorithms which compensate for sensed environmental conditions by modifying the tool path as well as the speed and feed rates. 
The functional capabilities of the proposed QIA system match the intended application domain of the Automated Manufacturing Programming Language Environment (AMPLE) system, developed at NIST. AMPLE is a uniform programming language environment for the construction of control interfaces to industrial manufacturing processes and an integrated system of software tools for translating product design and process planning specifications into equipment-level control programs. Since this programming language environment has been thoroughly described in other readily available publications [23-28], only a brief overview will be presented here. A detailed description of the programming language environment for QIA will appear in a separate report.

The need for self-monitoring and adaptive control requires a programming language environment to be expressive enough to contain an internal world model, including a representation of the workstation itself. This world model constitutes the core of the AMPLE system. Around the core is a loosely confederated, extensible bundle of software modules with which most programmers will interact. This two-level system architecture has two consequences. First, AMPLE contains explicit mechanisms for the definition of interfaces between each module and core, and also between several modules. Second, AMPLE has been designed to support a computing environment within which external software packages can be integrated $[25,26]$.

Some modules, such as those that provide access to core, will be required by almost all application programs. Other modules are tailored to more specific areas and have narrower scopes. Some of the widely used modules are a user interface for off-line programming, a communication module to provide a communication link between AMPLE and the workstation controllers, a process planning interface, and a real-time control interface to generate appropriate control code for the workstation's resident controllers in their native language [23]. Though there are many controller-level languages with which AMPLE may have to interface, the most important for QIA is the NC machine code language defined by the standard EIA RS-274D [21]. 
The general methodology to be followed in the QIA Project will be to describe the functional capabilities of the QIA system by developing an integrated system of AMPLE modules $[23,26]$. In all cases, the definition of each will consist of a description of (1) the operations that the module supplies, which may be understood to be a behavioral characterization of the system, (2) the various state variables that the system actually needs, and (3) the interface protocols that permit these modules to be interconnected, as well as their respective patterns of communication.

The QIA system is designed to be used in two different modes: off-line mode for verifying nominal part programs using the AMPLE NCVer module, and for resolving the programs into appropriate segments; and real-time mode for data acquisition and processing, and also for modifying segments to compensate for changes in the machining environment. Appropriate compensations can be done either by modifying the tool path in the next segment or by sending correction signals to the RTEC.

\subsubsection{OFF-LINE MODE}

The most important part of the off-line system is the verification and nominalization of NC part programs, which are always understood to be coded in conformity with EIA RS-274D.

Verification of part programs will be done by using the prototype NC verification module NCVer. This module does elementary syntactic errorchecking on part programs. If no fatal errors are detected, then NCVer generates a solid model of the part which would be produced by running the part program. This solid model is constructed by means of a specially crafted command interface from NCVer to a commercial solid modelling system, called GMS, produced by Interactive Computer Modelling, Inc. Specifically, given a solid model of a piece of stock or the in-process part to be machined as well as a description of the profile of the tool or tools to be used, NCVer converts the tool paths of the part program into geometric solids, usually by using the swept-volume method, and then sequentially removes each of these constructed volumes from the solid 
model of the part [29]. As noted in Sec. 2.3.4, no general agreement has yet been reached about the conditions which a part program has to satisfy in order to be accepted as nominal, but some points are obvious.

First, a nominal part program may only contain linear tool paths, and circular tool paths must be replaced by suitable linear approximations. This determination reduces one important source of non-repeatability by eliminating the system-specific methods of linear interpolation.

Second, a careful consideration of the segmentation problem suggests that the off-line programming system must be able to provide very high quality editing capabilities which not only help the programmer to modify interactively sets of part-program segments, but which also allow the user to maintain close connection with the original nominal part program, the manufacturing process plans, and all relevant design data. The programming system must also be able to maintain a very precise internal representation of the code so as to correctly propagate code changes throughout the set of segments. This is a specific case of the general problem of constraint propagation in complicated systems [30]. The off-line programming system must also supply meaningful defaults for the order in which the segments are to be passed to the NC machine controller for execution.

The structural organization of program segments is an important topic which can only be touched on here. Within the context of the AMPLE system, all programs are specific instances of abstract digraph type $[25,31]$. This combinatorial type is equivalent in expressive power to Petri nets. The nodes of the directed graph will be called boxes. The underlying model of execution requires that each box have some definite state. As with Petri nets, the significant aspects of the program net can be simulated by the motions of control tokens. When a program net is initiated, a single token is passed to the token-holder of all those boxes that have no immediate predecessors. These boxes are then said to be enabled. All subsequent markings of net are determined by the following enabling rule: once all operations in an activated box are successfully terminated, then one token is removed from that box's token holder, and 
one token is placed in the token-holders of all of the box's immediate successors.

The rules governing the activation of enabled boxes are more complicated. Any number, including all, of the enabled boxes may be activated concurrently. Hence, the only effective control that the programmer has over the order of execution is that which is forced by the enabling rule.

The off-line programming component should also provide computer assistance in ensuring the quality of finished and in-process parts by defining both manual and automated inspection procedures. Part inspection procedures will measure pre-defined dimensions of finished parts and in-process parts to determine whether all relevant tolerances have been met.

\subsubsection{REAL-TIME MODE}

Present interest in AMPLE-based systems naturally arises in connection with highly automated small-batch flexible manufacturing systems whose equipment is programmable and rapidly re-configurable to produce many different industrial products. To be economically feasible, such systems must be tightly controlled, which implies that all control programs must be verified before being used and that workstations must be equipped to monitor their own operating states and have some ability to adapt to changes in their operating environment [28]. But adaptive control is possible only if the workstations are equipped with sensors. Sensors may either be atomic, such as probes and temperature sensors, or more complicated sensory systems consisting of networks of simpler sensors [25].

Sensory data may directly affect the equipment-level control code which the real-time system must produce. For example, to produce a part that is within acceptable tolerances, it may be necessary to measure the temperature at selected points on the NC machine and then to compensate for the errors resulting from thermal drift by modifying the tool path 
in the NC part program [4,6]. This example suggests that interactions between off-line programmability and real-time control are complicated and require careful elaboration.

Assuming that a rationally segmented part program has been generated, then to further reduce systematic error requires us to compensate for changes in the machining environment. Such changes may only be detected by the real-time acquisition and processing of sensory data. Once detected, appropriate compensation can be done either by modifying the next part program segment, or by sending correction signals to the RTEC. As discussed in Sec. 2.2.2 real-time sensing will consist of four sensory modalities: thermal, vibration, force, and ultrasonic.

The AMPLE methodology recognizes that there are two very different kinds of issues that all real-time systems must resolve: (1) what operations or series of operations need to be performed, and (2) when must those operations be initiated, and perhaps more importantly, when must they be concluded.

That is, before one can adequately address the requirements for realtime processing, one should assume that the system has the capability of performing the necessary operations. In the present context, the assembly of the code to be executed and the specification of the precise order in which it is to be run is programmed off-line. More precisely, the effect of the off-line programming consists of a directed graph, each node of which represents one or more operations to be performed. The notion of a directed graph is especially useful in this context since it suggests that the nodes may be processed in several sequences, provided only that no node is initiated before its parents have finished. Nodes, all of whose parents have finished, are said to be enabled. Since it is usually assumed that graphs have such nice properties as being cycle-free, then it is always the case that there is a single well-defined set of enabled nodes. One could enforce some arbitrary selection rule during the offline programming phase, thereby guaranteeing that only one node is enabled at a time, but a more reasonable decision would be to allow the selection 
of the next enabled node to be made during the real-time phase of the system.

\subsection{INTEGRATION WITH THE AMRF}

The QIA architecture is to be integrated with the existing AMRF level structure and communications network.

The present AMRF architecture contains five levels as shown in Fig. $2.8[32,33]$. These are the facility, shop, cell, workstation, and equipment. Some control functions of each level are listed in Table 2.1. Quality related functions are also included there to support the overall quality control activities of the facility. The QIA architecture will primarily affect operations at the three lowest levels. In particular, Table 2.2 describes the functions of three key supporting elements of the QIA system at the cell level.

The quality controllers are to be inserted into the system at the equipment level just above the machine tool controller. The scheme for integrating them and the rest of the quality control system into the AMRF operation is shown in the network communication plan (Fig. 2.9). The horizontal, vertical, and inspection stations are already part of the AMRF command structure and are housed on the floor of the AMRF. The turning station is housed in a separate building and is not part of the AMRF.

During the machine tool characterization phase of the project and the initial machining experiments, the quality controllers will be connected to the quality monitor and the database (located with the file server in Fig. 2.9) via the OPENET/ETHERNET, and the initial control strategies will be developed on that system. All the commands to the machine tools will flow through the quality controllers independent of the workstation controllers so as not to disrupt the current AMRF software architecture and the AMRF MAP network during the testing phase. That is, the connections shown in Fig. 2.9 between the QC's and the workstation controllers will not be functioning. 


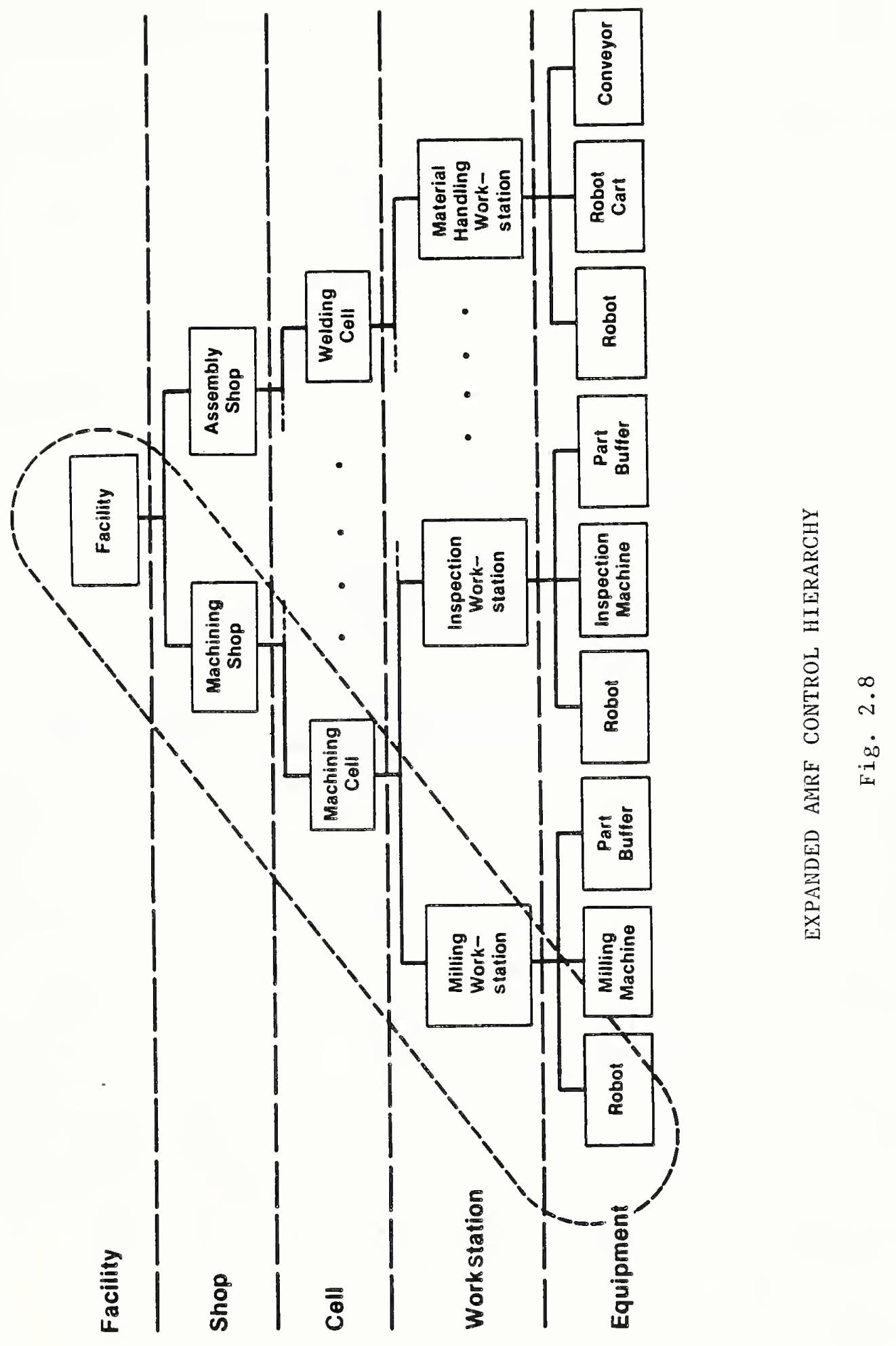


TABLE 2.1

AMRF ARCHITECTURE: LEVEL STRUCTURE AND FUNCTIONS

Facility

Coordinates the activities of design, manufacturing engineering and production control.

- Generates designs using design constraints and rules.

- Generates NC code from CAD drawings.

- Develops process plans based on process capability models.

Shop

- Sequences requests and schedules shop resources based on process evolution models.

- Maintains process evolution models based on archival quality data feedback from the shop floor.

- Receives requests with priorities and due dates from the facility level.

- Has overall responsibility for inventory control, tool management, capacity planning, and preventive maintenance.

- Coordinates activities of subordinate workstations to complete the jobs assigned by the shop.

- Develops schedules of anticipated start and finish times, and priorities for each job.

- Replans and reschedules to overcome developing conflicts and delays.

- Houses the quality monitoring system as one of its elements.

- Schedules and analyzes machine calibration. 
TABLE 2.1 (cont'd)

Workstation

- Generates sequences to perform the tasks assigned by the cell.

- Coordinates activities of materials handling, fixturing, and machining.

- Generates sequences by which the subordinates can complete each task.

- Directs machine calibration and setups for individual manufacturing processes.

Equipment

- Controls a single class of equipment such as a machine tool, robot, or materials handling device.

- Sequences through current tasks assigned by the workstation.

- Performs NC code correction in real-time or between process steps.

- Performs machine calibration and setup. 
TABLE 2.2

SUPPORTING ELEMENTS FOR QIA PROJECT AT CELL LEVEL

ELEMENT

Quality Monitor

Quality Network

Database

\section{FUNCTIONS}

- Performs comparison of NC Codes with CAD designs.

- Gives user access to the database regarding dimensional, surface finish, and other data on parts.

- Displays graphs, error maps, and control charts of the dimensional and surface finish data.

- Houses theoretical models of the machines (e.g., results of finite element analyses) and displays graph and error maps of this information.

- Compares theoretical and experimental error maps.

- Provides the link between QC's and shop quality monitor and other quality-related workstations.

- Interfaces to the database containing part and process information. Some of this information is archival; some of it is temporary.

- Provides a gateway to the AMRF and interacts with process planning there. Certain process planning steps are in the domain of the AMRF, but correction factors to enhance the accuracy are provided through the quality network.

- Houses the quality data. Access to the data is organized in several ways. To the user these may look like different types of data files.

Example:

a) Part/Process Active Data File:

- Part ID \#, number of parts

- Nominal and "corrected" NC part programs EIA RS-274D 
Database (cont'd)

- Tooling list

- Fixturing instructions

- Fixturing data

- Fixturing diagram

- Tool setting data

- Tool time in cut - Expected

$$
\text { - Actual }
$$

- Estimates of remaining tool life based on above data.

- A developing history of operator comments. This comprises a maintenance function and an expert system function.

- Temperature records for up to 40 sensors for each machine, with data included on the time intervals used.

- Probing data and probing scenarios. This includes probe-proof data (i.e. configurations, shapes, dimensional info, and diameters of probes).

- Materials

- Preprocessing instructions - ex: steps for preparation of the blank, heat treatment, etc.

b) Part Data File:

- Part specific data extracted from file (a).

c) Process Data File:

- Machine specific data extracted from file (a).

d) Part and Process Archival Data File:

- Product Definition: part geometry, material, and tolerances in PDES Format.

- Nominal uncorrected EIA RS-274D inspection code.

- Quality control data or inspection results (ex., geometry and surface finish data). 


\section{NETWORK COMMUNICATION PLAN}
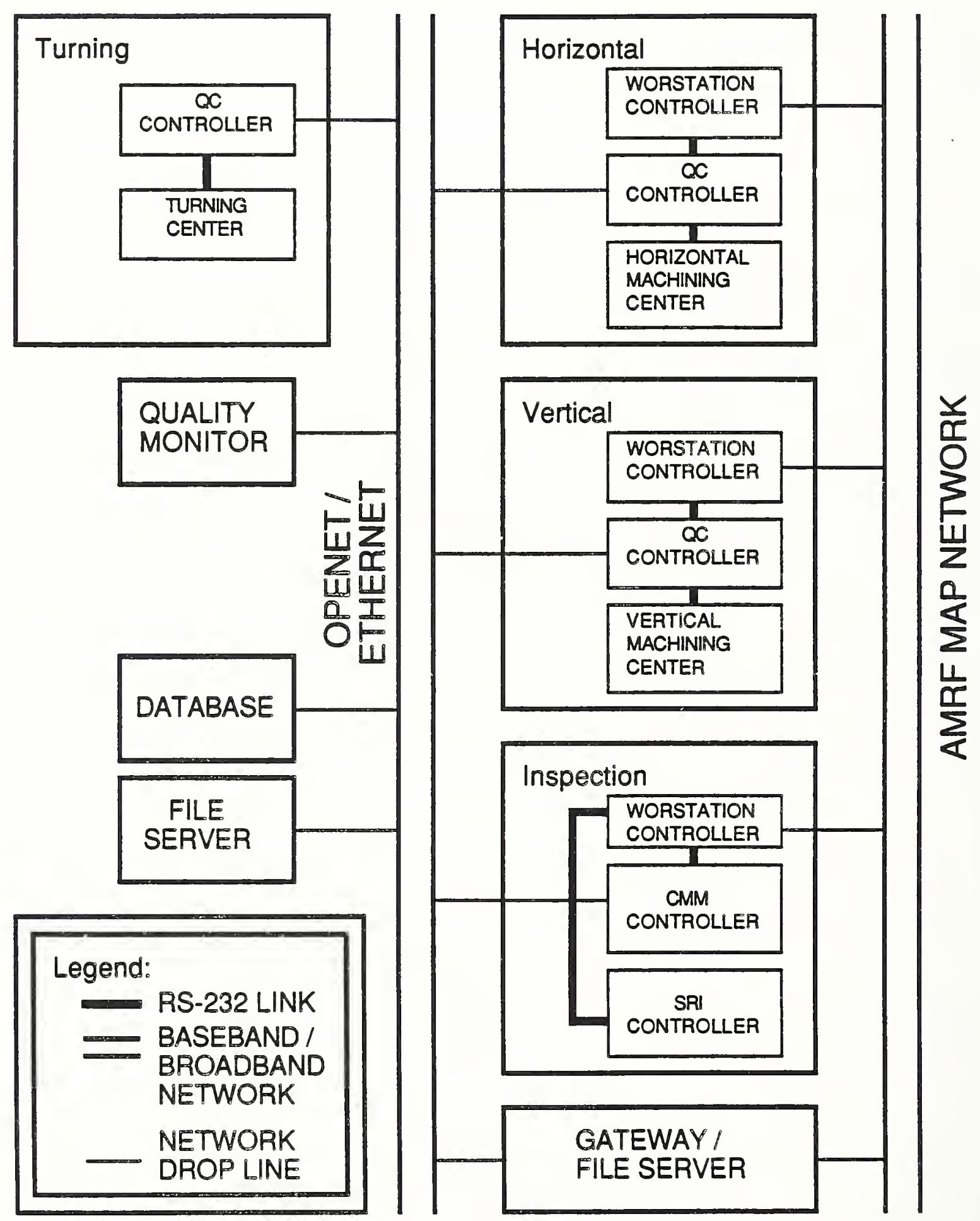

Fig. 2.9 
Once the initial experiments have been completed and the operation of the control system has been shaken down, the OPENET system will be connected to the AMRF net via the Gateway and two of the quality controllers will be integrated into the AMRF netowrk and the hierarchical command structure of the AMRF via connection to the vertical and horizontal workstation controllers. This hardware change will be accompanied by software modifications to the two workstation controllers to accommodate the new quality control and monitoring functions. 


\title{
3. MACHINE TOOL CHARACTERIZATION
}

\author{
F.F. Rudder, Jr.
}

\subsection{OVERVIEW}

The purpose of characterizing a machine tool is to determine the tool or cutter positioning errors relative to the workpiece. These positioning errors establish the machine tool accuracy and the precision of the workpiece dimensions.

The total positioning errors of the tool relative to the workpiece are the result of a complex interaction among the machine tool elements, the physical machining operations, and the dynamic environment within which the machine tool operates. Due to the complexity of this interaction, it is necessary to determine the components of the total positioning errors and the dependence of these components upon the physical machining operations and the resulting dynamic environmental conditions. With this knowledge it may then be possible to characterize the machine tool and improve the machining accuracy.

Among the major factors affecting the positioning errors are the geometric errors of the machine tool, static deformation of the machine tool when loaded by large workpieces, and thermal effects on the geometric errors. Geometric errors are caused by unwanted motions of the machine elements such as carriages, cross-slides, and worktables. These unwanted motions occur because of geometric imperfections and misalignments. Heat generated by the machine tool and the cutting operation causes temperature changes of the machine tool elements and environment. Due to the complex geometry of the machine structure, concentrated heat sources, such as the drive motors and the spindle bearings, create thermal gradients along the machine structure. Spindle growth, lead-screw expansion, and a significant part of the machine structure deformation are the results of these temperature changes and gradients $[4,34]$. 
Improvement in machining accuracy is accomplished by introducing compensation for the tool path movement. The compensation considered here focuses attention upon the geometric and thermally-induced error for three types of CNC machine tools: a three-axis vertical spindle machining center, a three-axis horizontal spindle machining center, and a turning center. Hence, the task requires the experimental determination of geometric errors and their dependence upon the thermal environment of the machine tool.

\subsection{MODEL FOR ERROR COMPENSATION}

Error compensation requires a quantitative description of the spatial relationship between the cutting tool and the workpiece. Due to the complex mechanical systems, the diversity of machine tool configurations, and the dynamic environmental conditions, it is necessary to utilize a model and/or methodology to describe the spatial characteristics of a machine tool and to determine the errox experimentally. The model guides the experimental plan, the data acquisition and analysis, and the error estimates. The error estimates provide the quantitative description of the error compensation.

The use of transformation matrices to define the kinematic description of a machine tool is well established $[4-6,35,36]$. These transformation matrices define the orientation of each axis of a machine tool relative to each other and to the machine tool reference frame. The elements defining each matrix are the position exrors of the axis, the intended movement along the axis, and the geometry describing the machine tool component. The compound displacement resulting from kinematically-related components is then obtained by matrix multiplication. In this manner, the locations of the workpiece and the cutting tool are established relative to the machine tool reference frame and the total position error is estimated.

This model requires the position errors for each axis (including the effect of thermal gradients, etc.) as data to establish the required 
compensation. The measurements of these position errors is an experimental task that is now described.

\subsection{EXPERIMENTAL BASIS FOR ESTIMATING POSITIONING ERRORS}

\subsubsection{DATA ACQUISITION REQUIREMENTS}

The data requirements for estimating machine tool positioning errors necessitate the development of an empirical data base that combines commanded machine tool position, absolute machine tool position, and machine tool environmental condition. The commanded machine tool position is determined by the part program being executed and is read directly into the data base from the machine tool registers. The absolute machine tool position is read directly into the data base from a laser interferometer controlled by a PC. The machine tool environmental condition is based upon sensor outputs located on the machine tool. Presently, thermocouples are the only sensors installed upon the machine tools.

The data acquisition is conducted in an orderly sequence of events for each axis of the machine tool. This sequence of events is illustrated in Fig. 3.1. The resulting data set represents beginning temperature, machine tool position data, and ending temperature. This data set is followed by a constant cycle of machine tool operation the purpose of which is to alter the machine tool temperature. The data acquisition is then repeated.

The "AXIS POSITION PROGRAM" indicated in Fig. 3.1 is a sequence of commanded moves (in absolute machine tool coordinates) for a given axis. This is a part program resident in the machine tool controller. This part program composes a sequence of moves beginning at one limit of the axis, extending to the opposite limit, and returning to the beginning position. The axis travel is divided into uniform discrete intervals that define the "commanded" positions. The machine tool pauses at each commanded position until the machine tool position and the laser position have been logged 


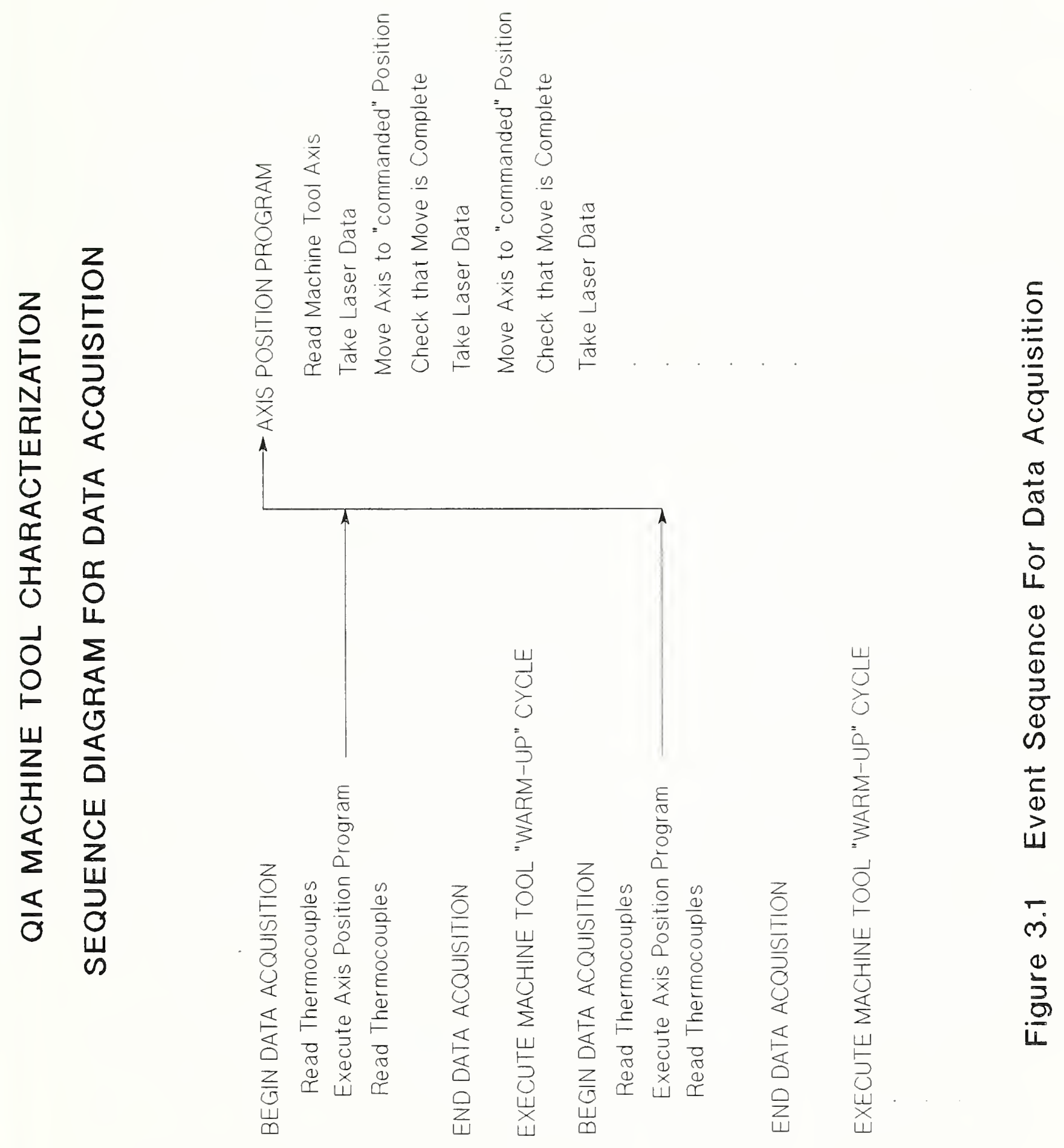


into the data base. The machine tool is then moved to the next commanded position.

\subsubsection{DATA ACQUISITION SYSTEM}

The above data acquisition requirements identify the need for an automated data acquisition system. Such a system, based upon a PC controller, is illustrated in Fig. 3.2. Using a resident program, the controller orchestrates the sequence of events described previously to log data in a orderly fashion. The data acquisition system illustrated in Fig. 3.2 may also be utilized for other purposes such as logging temperature profiles during machine tool operation. This use will be described subsequently.

\subsubsection{THERMOCOUPLE INSTALLATION}

Temperature sensing on all of the machine tools is accomplished using type $\mathrm{T}$ thermocouples. Figure 3.3 illustrates the fabrication sequence utilized for all thermocouples. Following fabrication, all thermocouples were calibrated, using the data acquisition system, at $0^{\circ} \mathrm{C}$ (ice point) and at $29.77^{\circ} \mathrm{C}$ (Gallium-Cell) to establish the complete circuit from the thermocouple junction to the disc storage of the data.

Following calibration, the thermocouples were installed on the machine tools at selected locations. The specific locations will be described subsequently. The detailed "sandwich" installation utilized for each thermocouple is illustrated in Fig. 3.4. For each thermocouple, the wire was coiled about the copper disc as illustrated. The disc was then bonded to the appropriate location on the machine tool using a special-purpose epoxy cement characterized by its high electrical resistance and high thermal conductivity. Curing time for this epoxy is 24 hours at room temperature. After curing, the thermocouple junction and coil were covered with a sticky-backed foam pad. The purpose of this pad is to provide thermal insulation of the junction from environmental factors exterior to the machine tool surface. The complete "sandwich" was then 


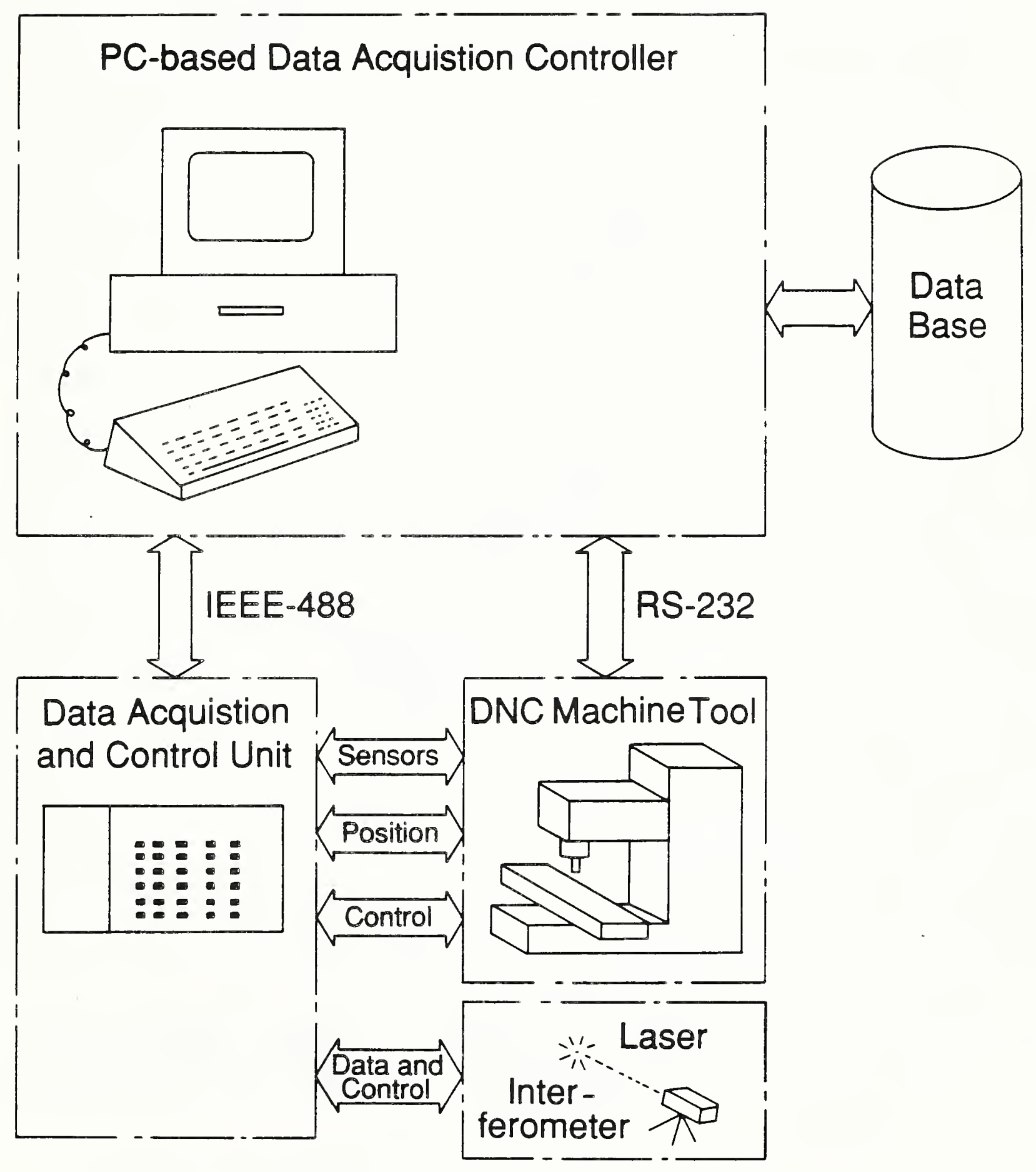

Data Acquisition System

Fig. 3.2 


\section{Thermocouple Fabrication Sequence}
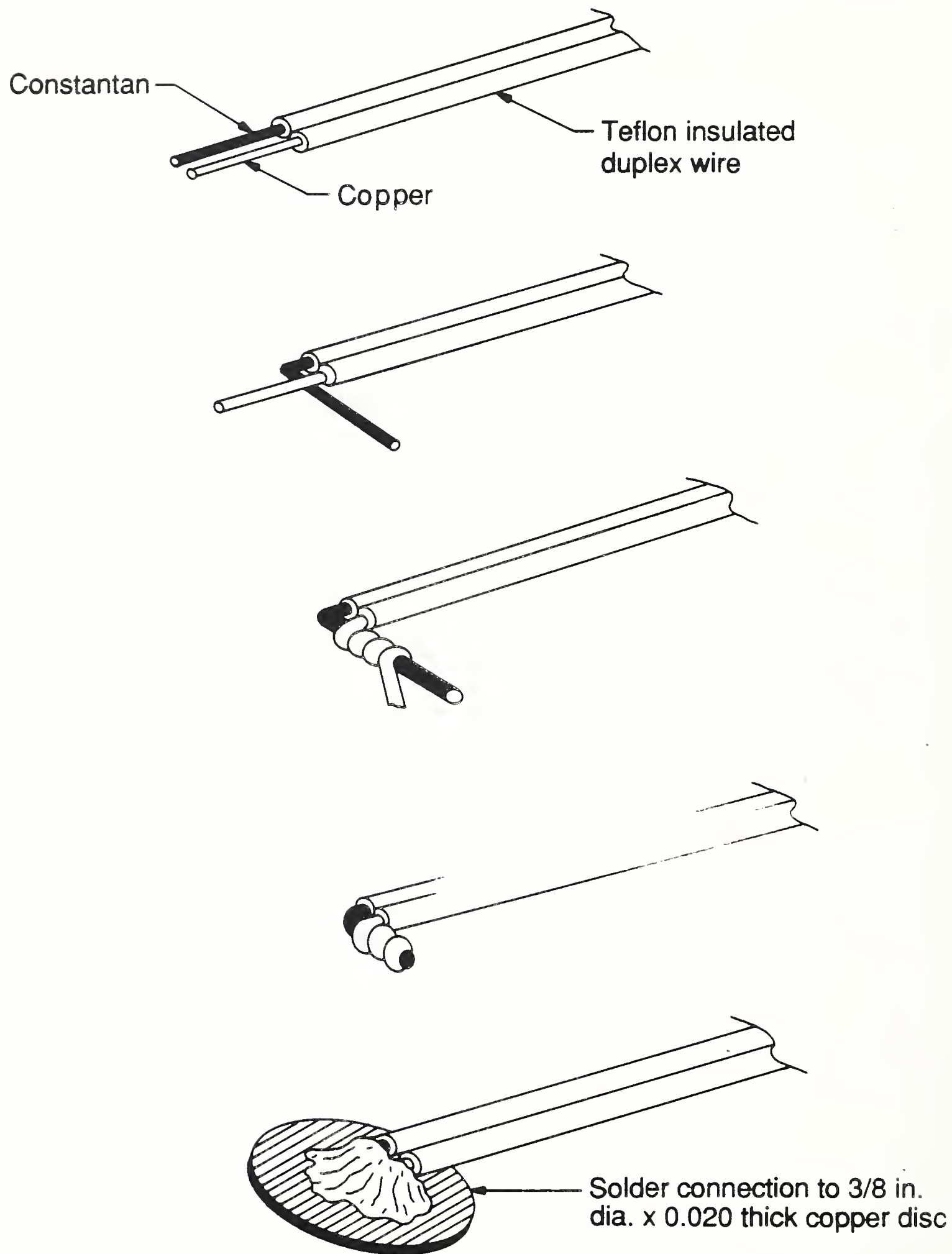

Thermocouple Fabrication Sequence

Fig. 3.3 


\section{Typical Thermocouple Installation}

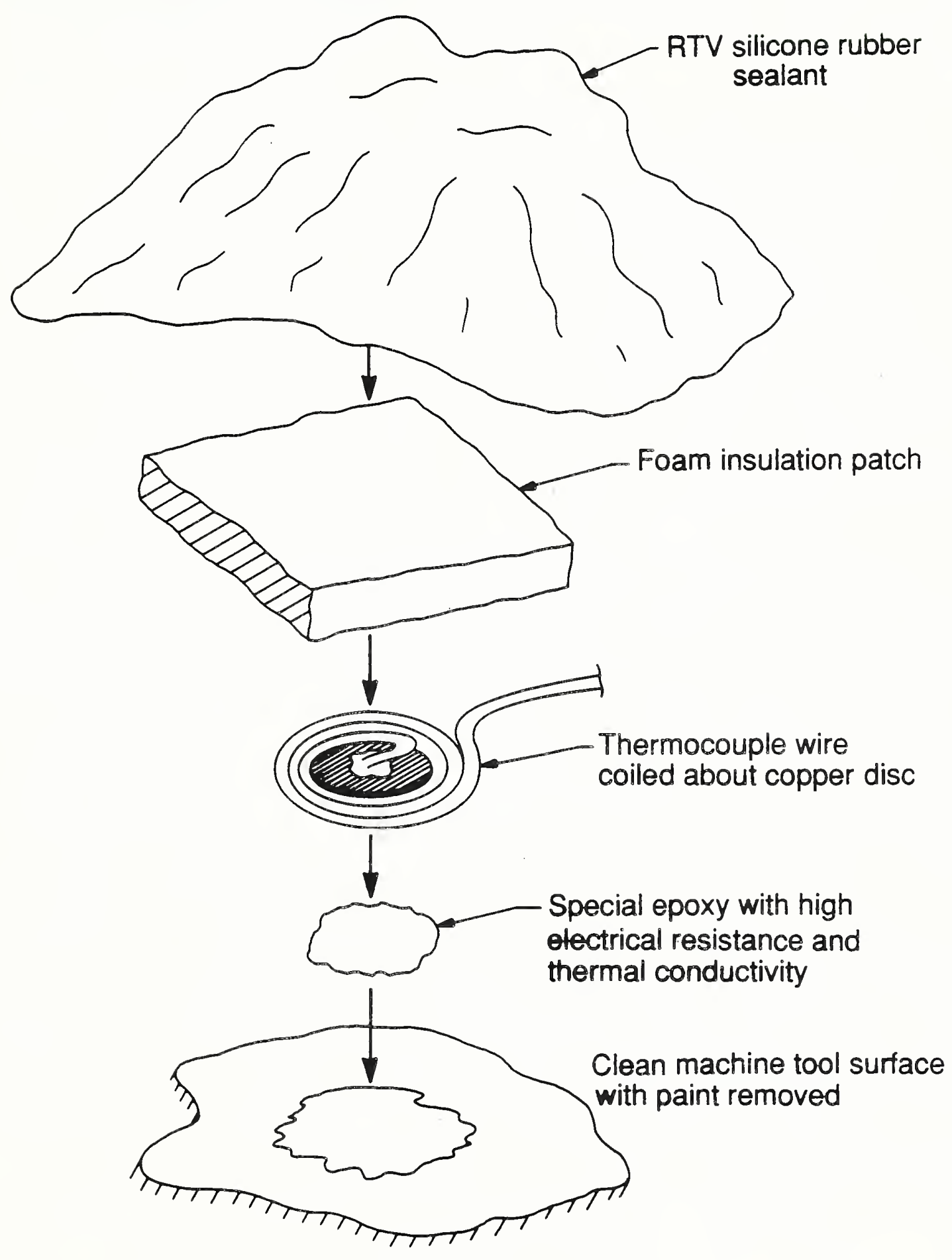

Fig. 3.4 
covered with RTV silicone rubber sealant to provide resistance to direct exposure of the junction from cutting fluids, oil, etc. The thermocouple wire was then routed and attached to the machine tool, as appropriate, to ensure that any machine tool movement did not interfere with the installation.

For the three machine tools utilized in this project, over 120 thermocouples were installed requiring more than 2400 feet of wire. The specific thermocouple locations for each machine tool are illustrated in Fig. 3.5 through Fig. 3.7. Each location is identified by a dark spot and a number. The description of each location is presented in Tables 3.1 through 3.3. Each numbered location corresponds to a data channel number in the data acquisition unit.

\subsubsection{DATA LOGGING}

The data acquisition system described previously may be used to log time series data during machining operations. One initial use of the system has been to log temperature profiles or histories to observe the range of temperatures encountered during machining. Another initial use of the system has been to log temperature profiles for parametric evaluation of spindle speed and axis feed rate for defining a "warm up" cycle to be used in the measurement of position errors as related to thermal environment.

Figure 3.8 presents a single machining cycle for the vertical machining center illustrating the response of four thermocouples. The thermocouples are identified in Fig. 3.5 and Table 3.1 and the machining was initiated from a cold machine condition. Figure 3.8 illustrates the non-equilibrium thermal conditions that occur during machining. Such conditions imply that the data acquisition and the subsequent (statistical) data analysis more than likely must account for a time lag effect in addition to the consideration of the instantaneous temperature at a location. Since both heating and cooling will be occurring during machining, it is likely that a thermal time constant for each thermocouple 


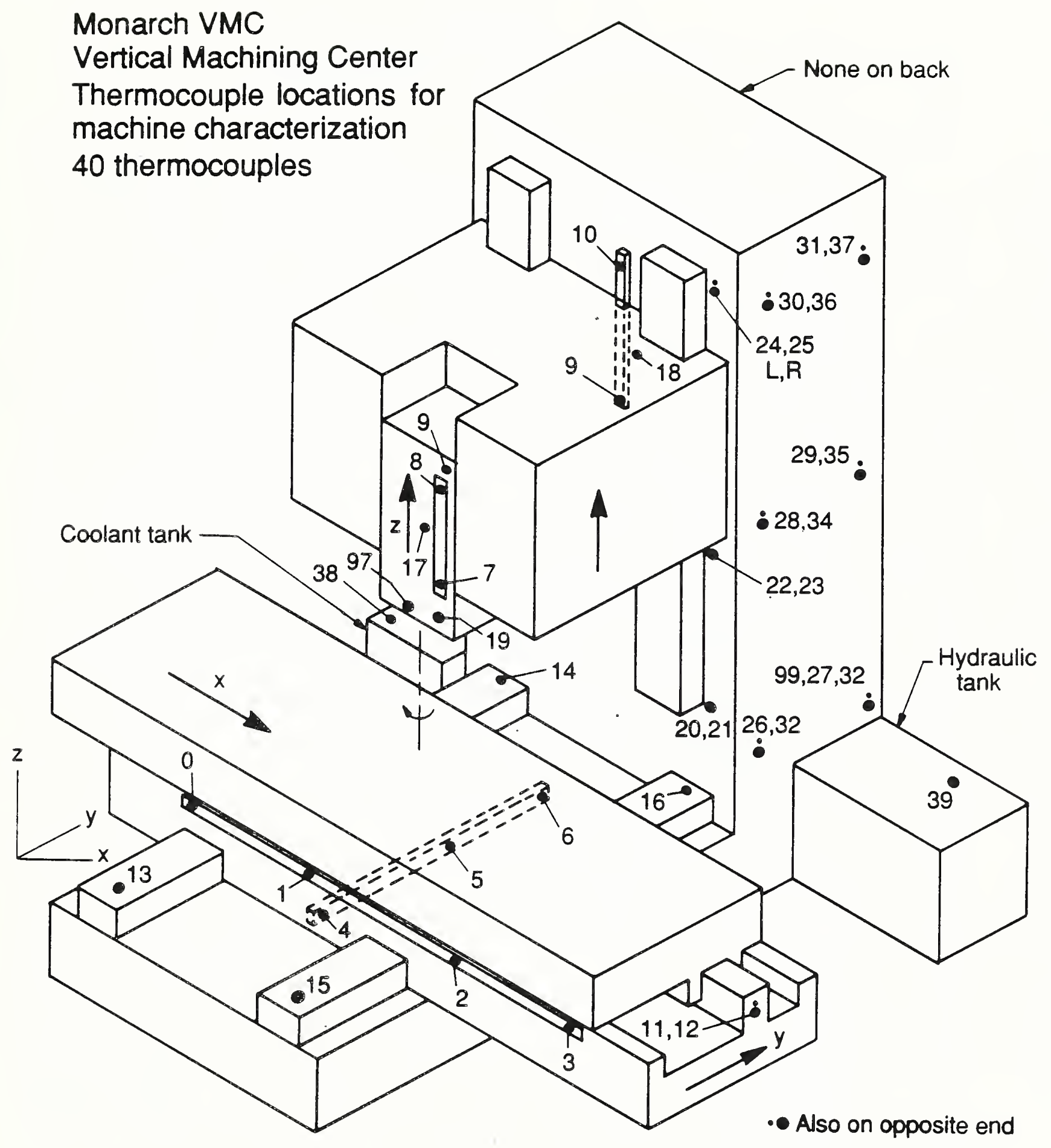

Fig. 3.5 
TABLE 3.1

THERMCOUPLE LOGATIONS ON MONARCH VMC

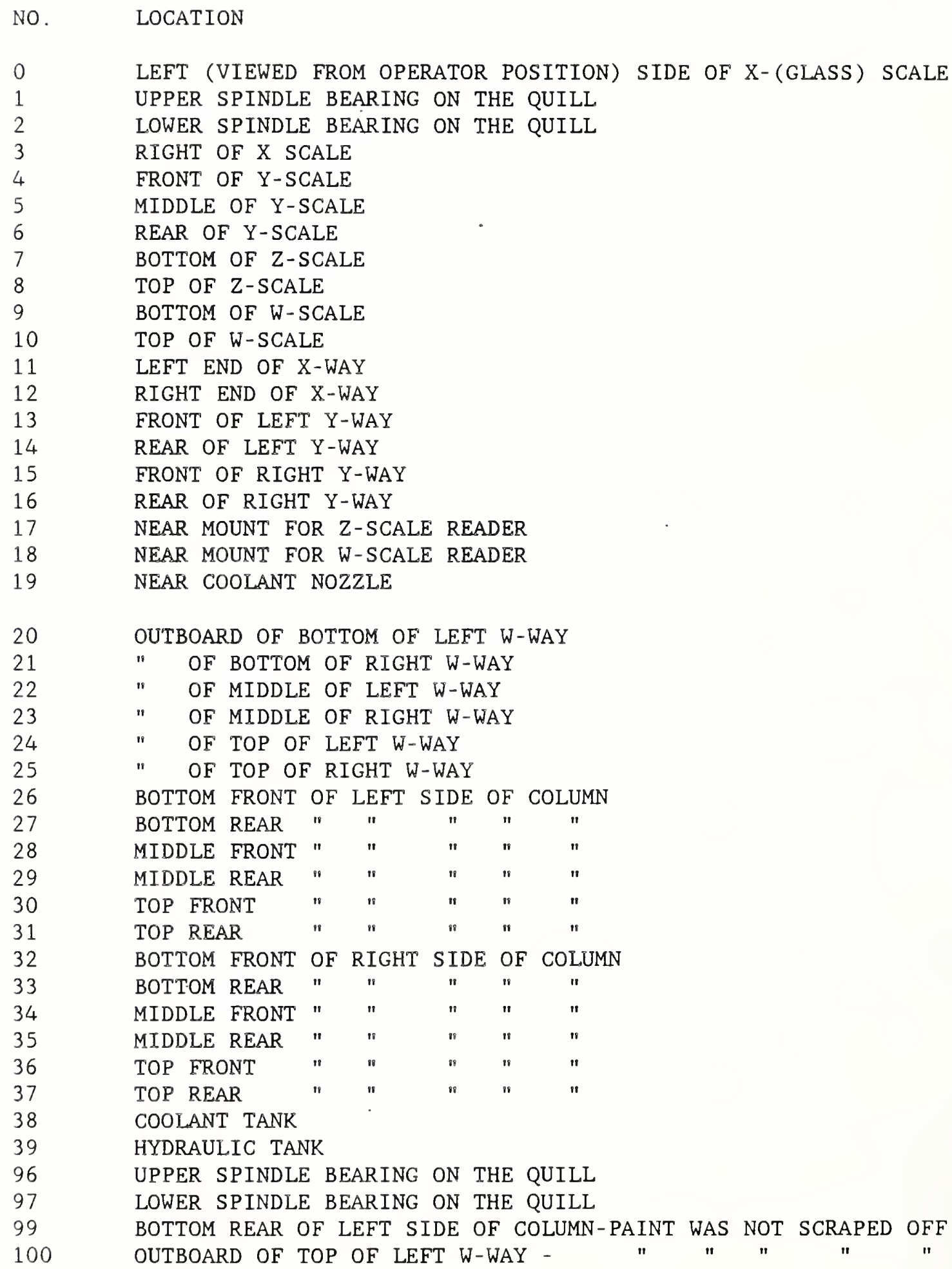




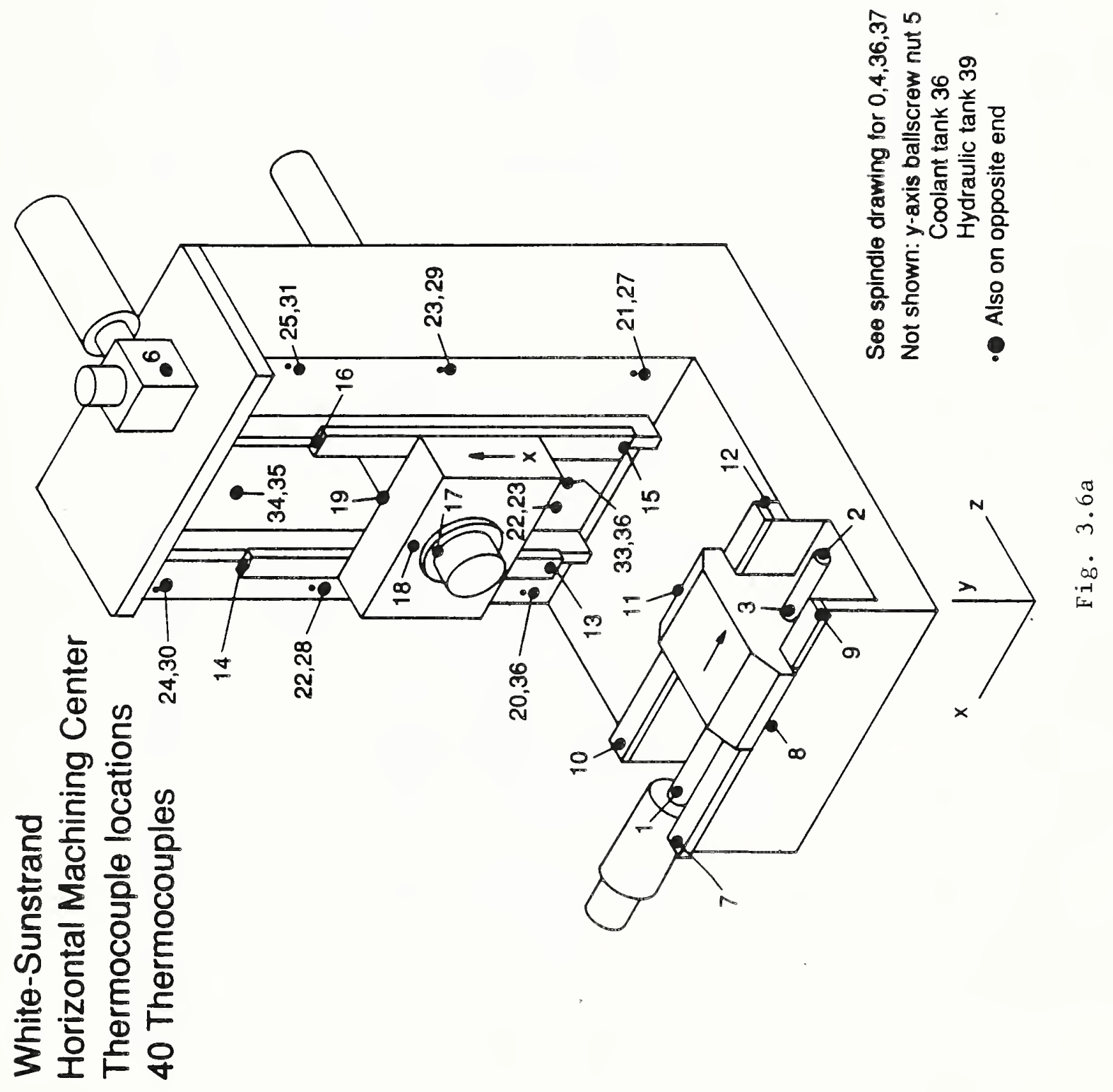




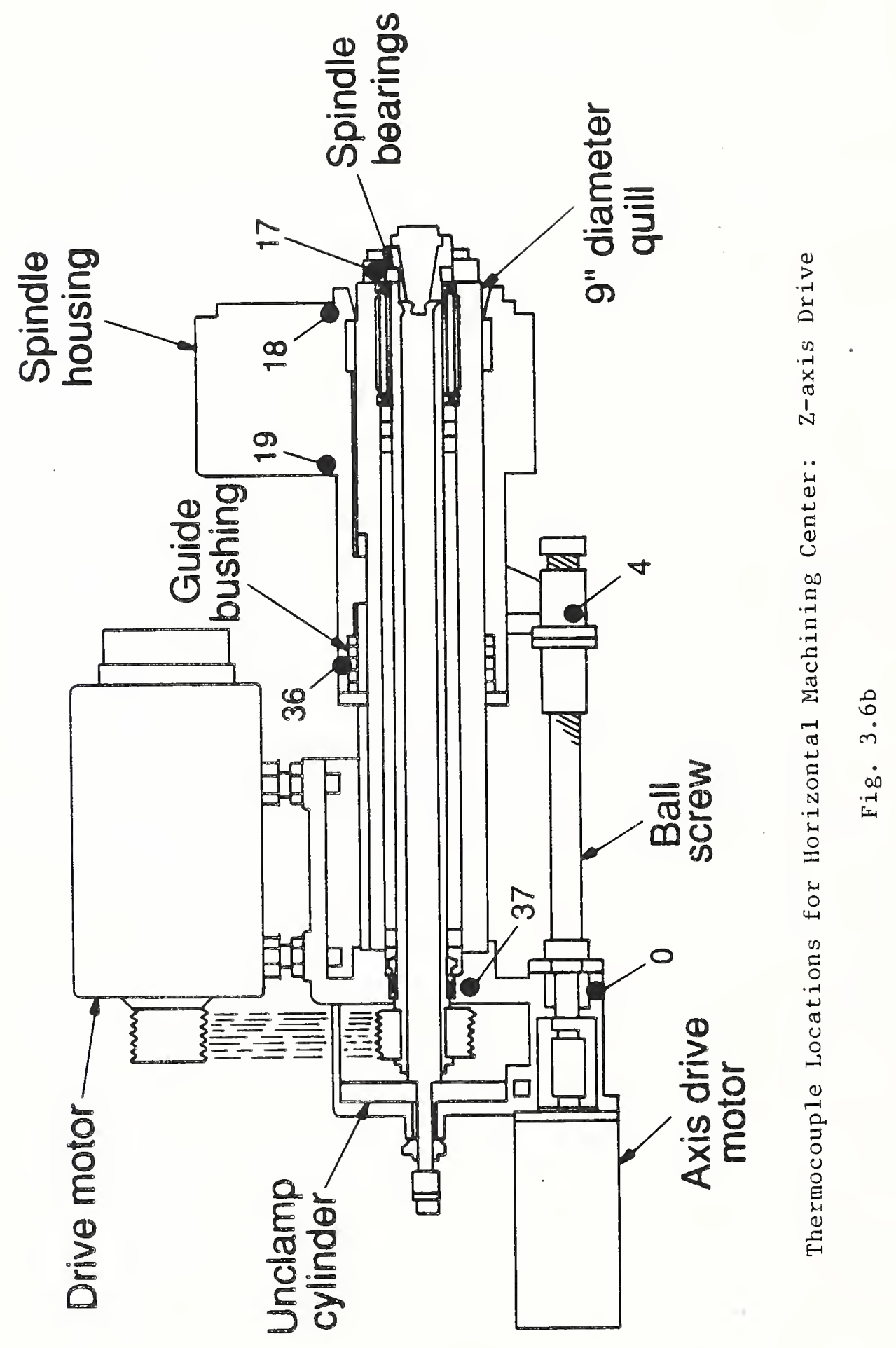


TABLE 3.2

THERMOCOUPLE LOCATIONS ON WHITE-SUNSTRAND HWS

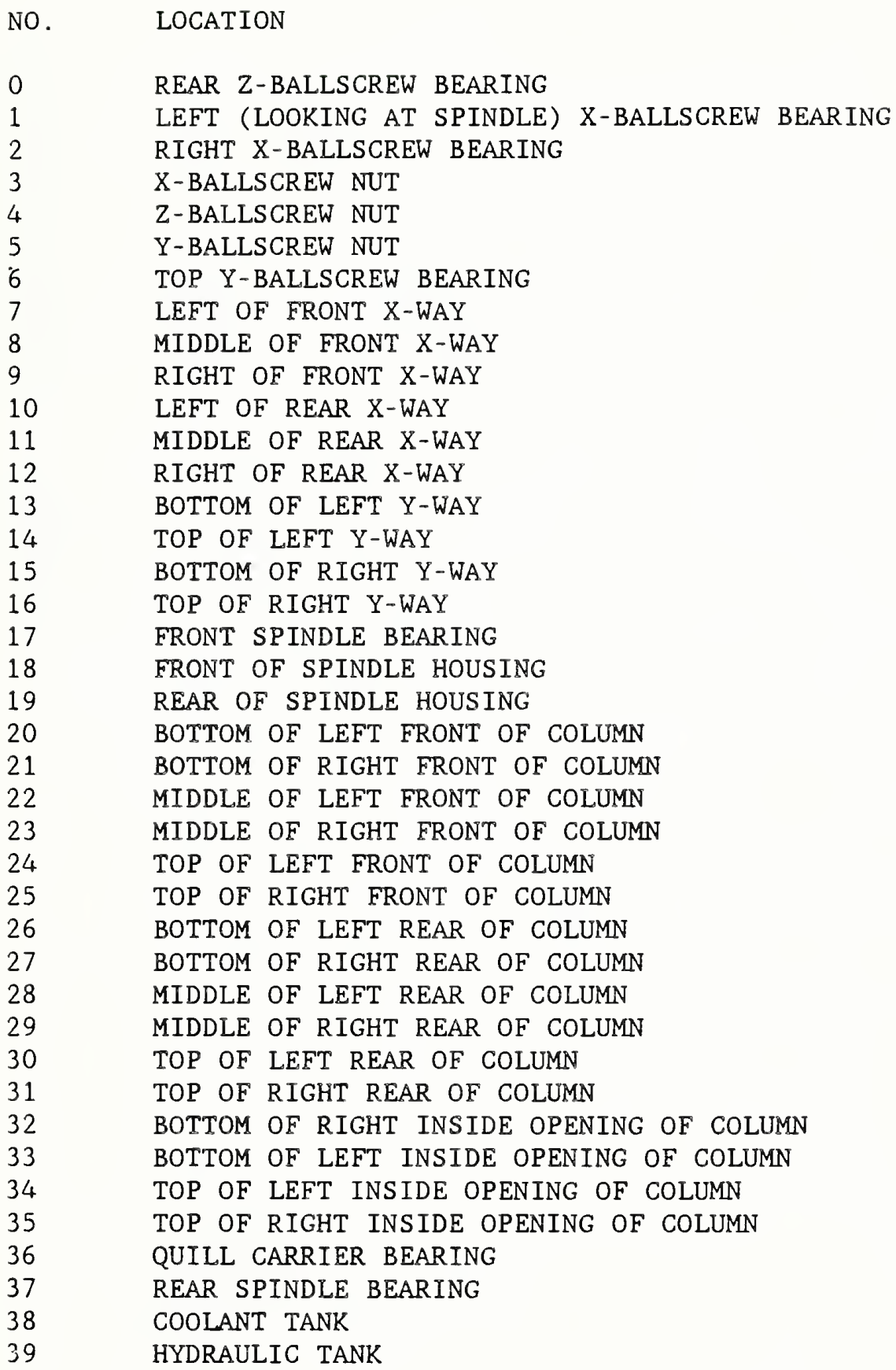




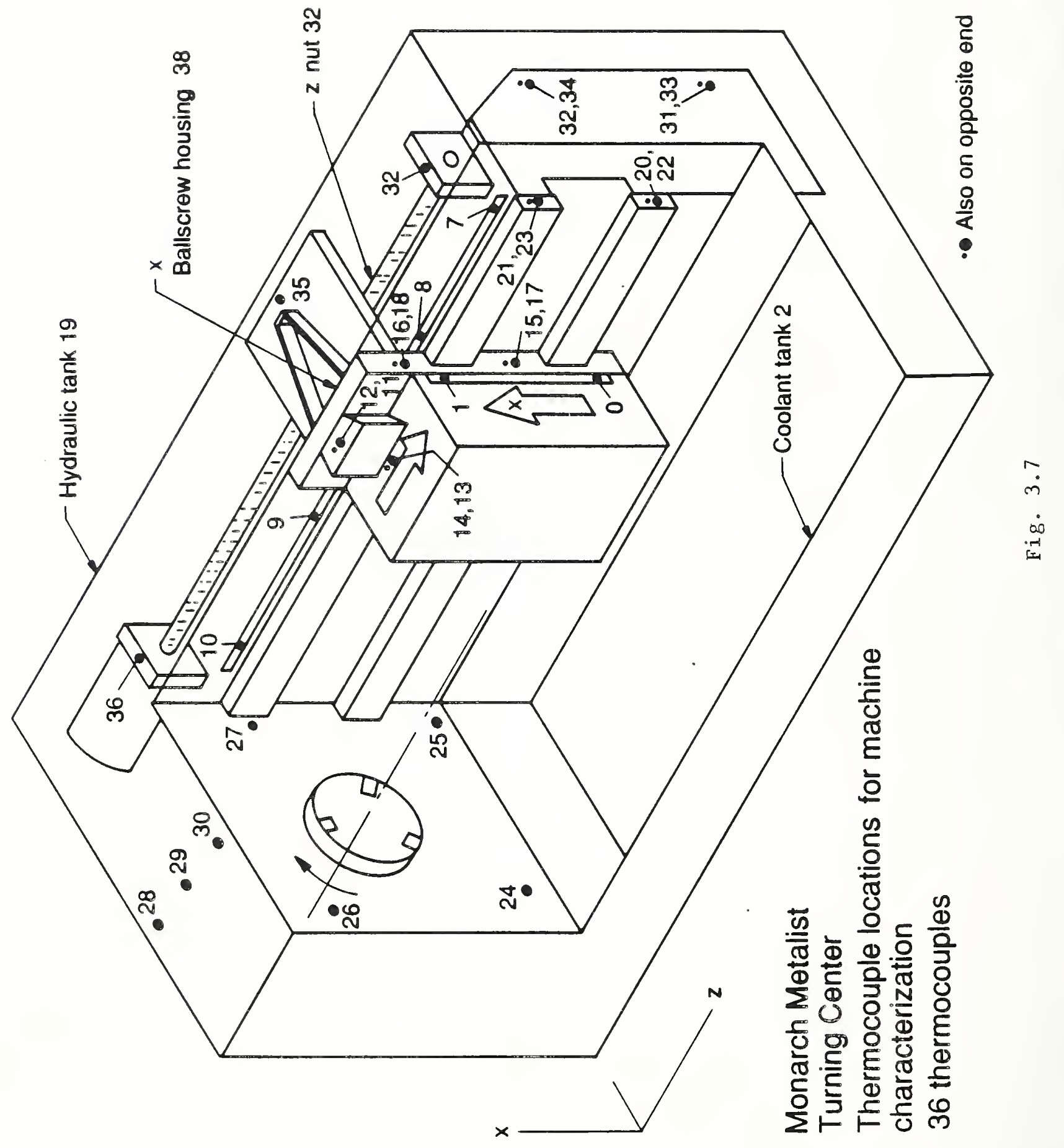


NO. LOCATION

$\begin{array}{lll}0 & \text { BOTTOM OF X-(GLASS) } & \text { SCALE } \\ 1 & \text { TOP OF X-SCALE } & \\ 2 & \text { COOLANT TANK } . \\ 3 & \text { NOT USED } \\ 4 & \text { " " " } \\ 5 & \text { " " }\end{array}$

RIGHT OF Z-SCALE

RIGHT CENTER OF Z-SCALE

LEFT CENTER OF Z-SCALE

LEFT OF Z-SCALE

TOP OF $X$-WAY

BOTTOM OF X-WAY

TOP OF X-HEAD

BOTTOM OF $X$-HEAD

BOTTOM LEFT OF Z-SLIDE

TOP LEFT OF Z-SLIDE

BOTTOM RIGHT OF Z-SLIDE

TOP RIGHT OF Z-SLIDE

HYDRAULIC TANK

LEFT END OF LOWER Z-WAY

LEFT END OF UPPER Z-WAY

RIGHT END OF LOWER Z-WAY

RIGHT END OF UPPER Z-WAY

LOWER FRONT OF SPINDLE HEAD

LOWER REAR OF SPINDLE HEAD

UPPER FRONT OF SPINDLE HEAD

UPPER REAR OF SPINDLE HEAD

LEFT OF TOP OF " "

MIDDLE OF TOP OF " "

RIGHT OF TOP OF " "

BOTTOM LEFT OF BED

TOP LEFT OF BED

BOTTOM RIGHT OF BED

TOP RIGHT OF BED

NEAR X-DRIVE MOTOR SHAFT BEARING

LEFT Z-BALLSCREW BEARING

RIGHT Z-BALLSCREW BEARING

$X$-BALLSCREW HOUSING

Z-BALLSCREW NUT 


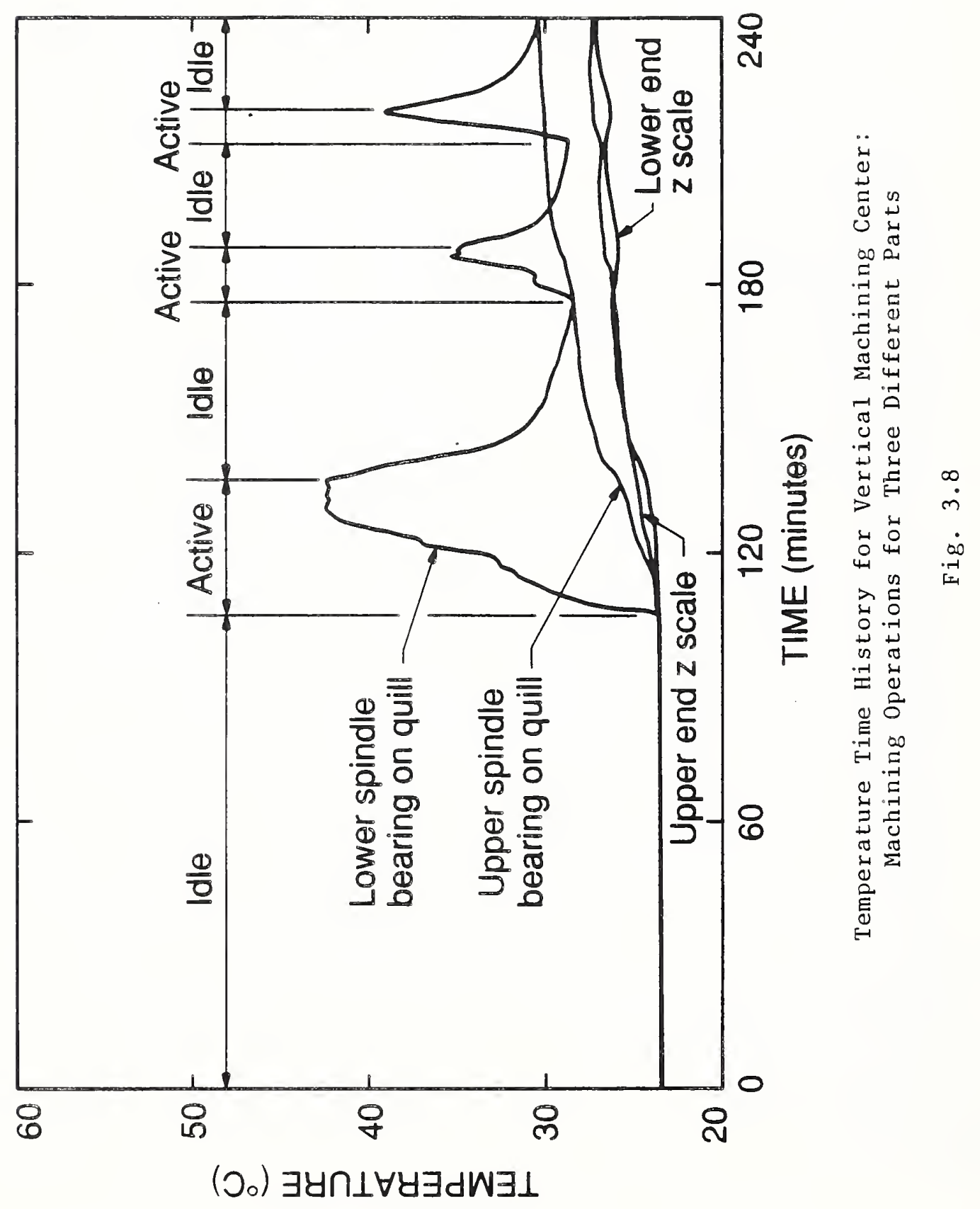


location may be an important consideration. These time constants must be determined experimentally.

Figures 3.9 and 3.10 are temperature profiles for the vertical machining center for two different spindle speeds during a sixteen hour warm up/cool down cycle. The five temperature profiles shown in figures $3.8,3.9$, and 3.10 were obtained from the same four thermocouples. The warm up/cool down cycle used does not represent actual machining since the tool was "cutting air". The purpose of these experiments was to determine the time required for the thermocouples to reach thermal equilibrium during the heating phase and to compare the cooling characteristics of various elements of the machine tool during the cooling phase.

\subsubsection{MEASUREMENT OF POSITIONING ERRORS}

For each of the three machine tools included in this project, an extensive experimental program must be conducted to measure the tool-tip positioning errors. Since each of the machine tools possess a different kinematic relationship between the workpiece and the tool tip, the details of the measurements will vary for each machine tool. However, the overall approach is the same for all machines.

Table 3.4 lists the different measurements required to obtain the machine tool positioning errors for the vertical machining center. The horizontal machining center will have similar measurement requirements, but the turning machining center will not require measurements related to $y$-axis movement. For each measurement, the technique to be utilized is listed. Except for the error due to roll motion along an axis, the position errors will be measured using a laser interferometer with appropriate optics. For the roll of horizontal axes, electronic levels will be utilized. For the roll of vertical axes, an offset spindle mounted LVDT probe will be utilized with table-mounted straight edges. This approach assumes that the machine tool elements behave as rigid bodies kinematically linked to each other. The nomenclature for the rigid body motion is illustrated in Fig. 3.11 . 


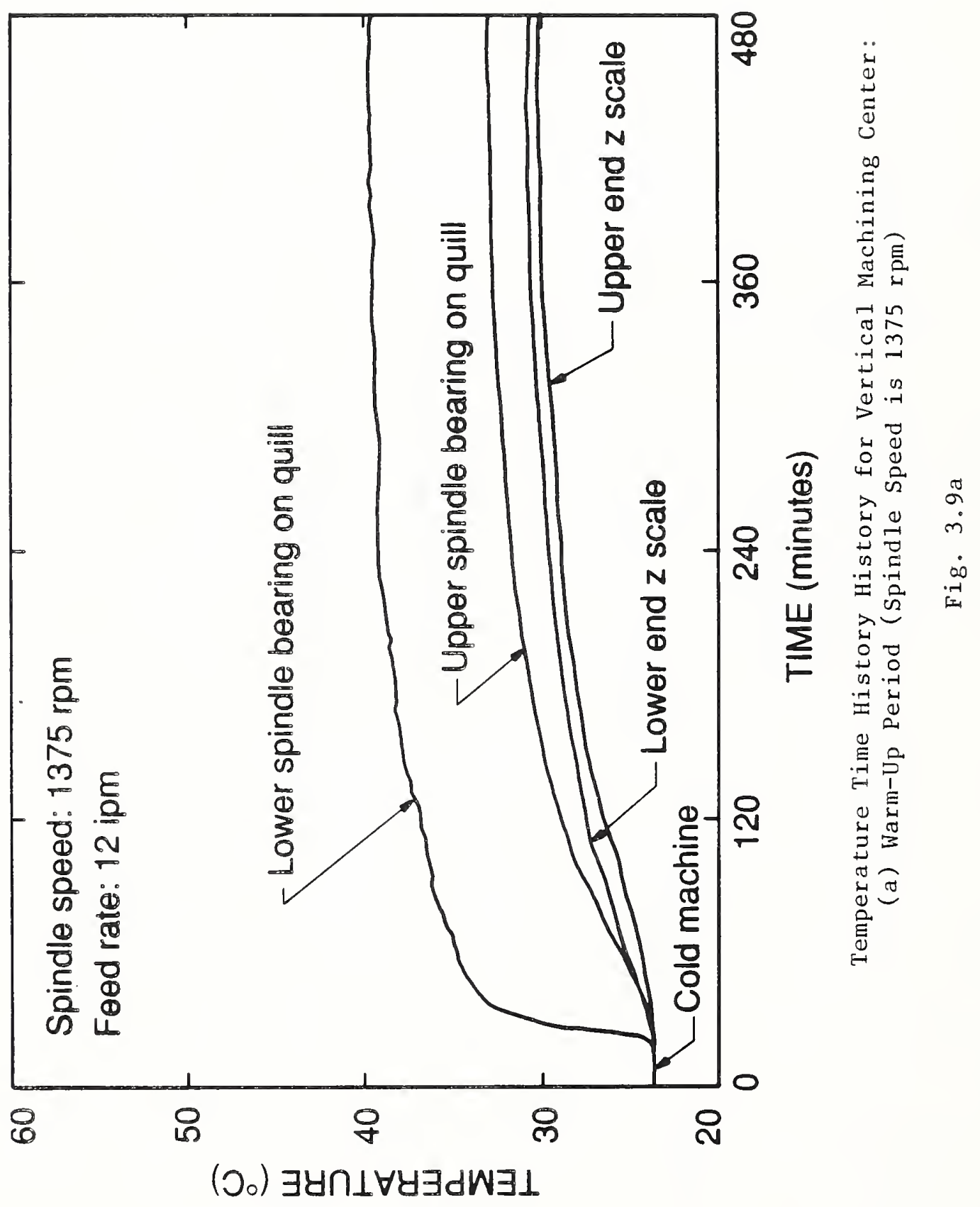




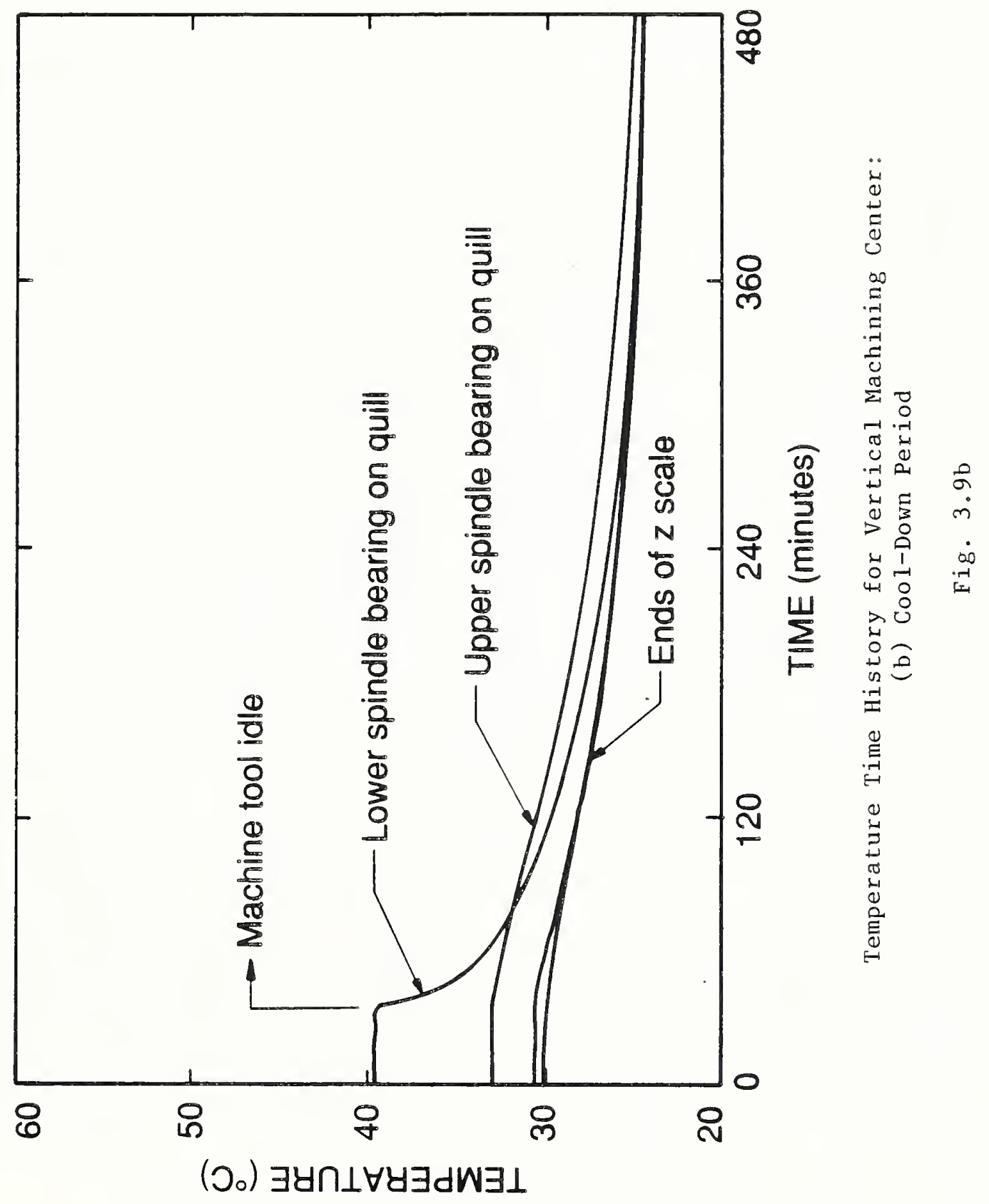




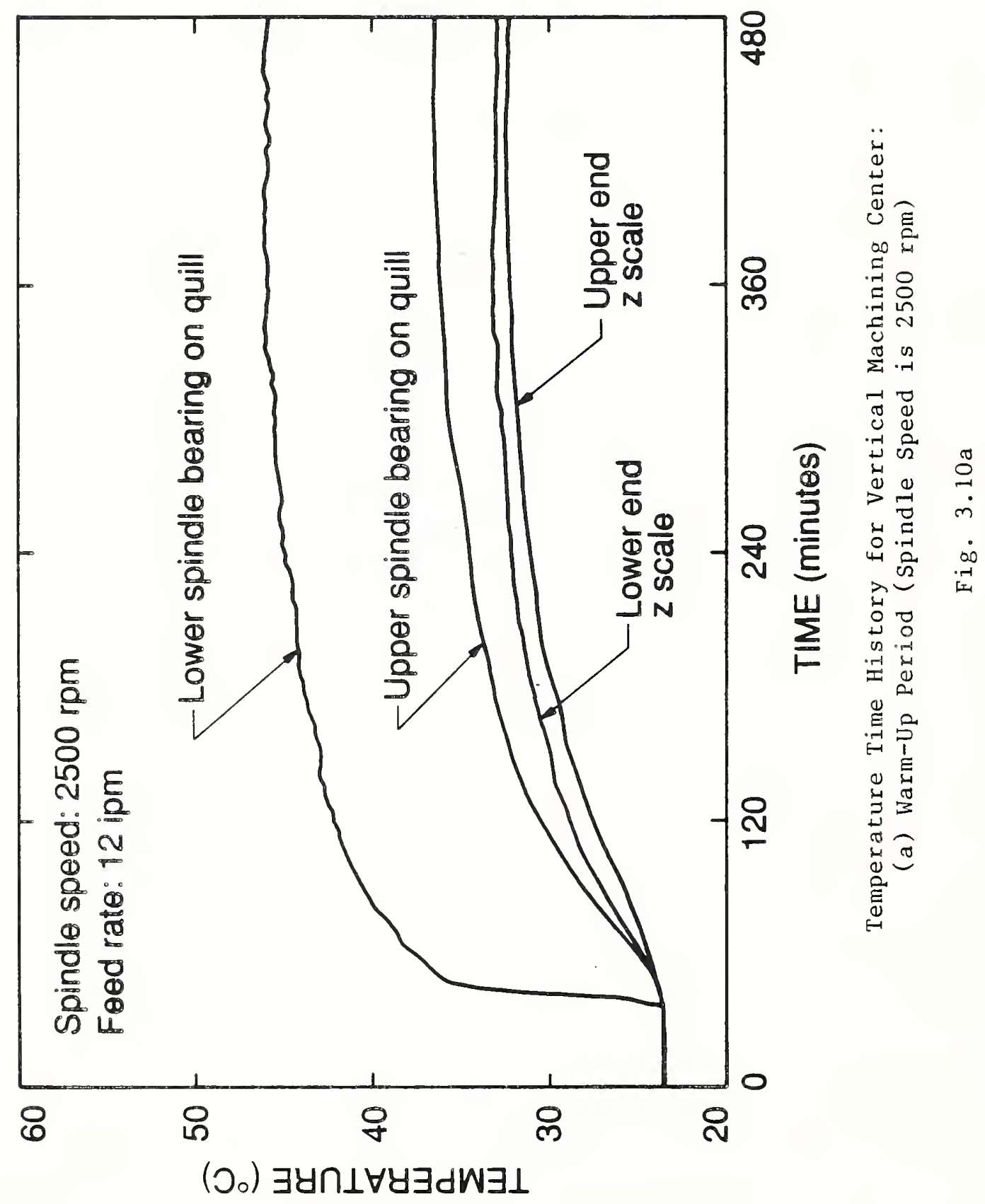




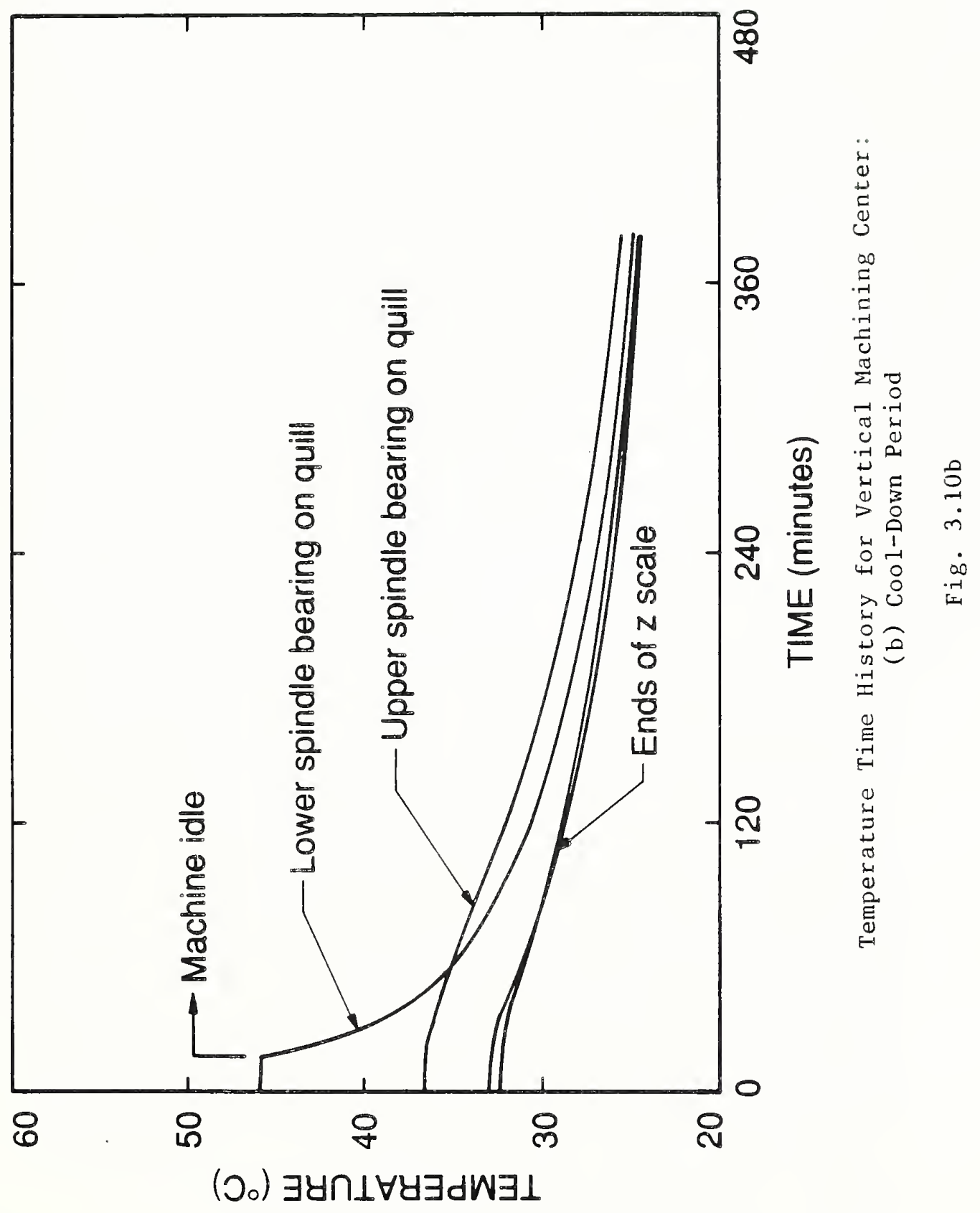


TABLE 3.4

MEASUREMENTS REQUTRED FOR POSITIONING ERRORS

\begin{tabular}{|c|c|c|c|}
\hline Measurement & Axis on Plane & Method & Comment \\
\hline Distance & All Axes & Laser Interferometer & \\
\hline Pitch & All Axes & Laser Interferometer & \\
\hline Yaw & All Axes & Laser Interferometer & \\
\hline $\begin{array}{l}\text { Vertical \& } \\
\text { Horizontal } \\
\text { Straightness }\end{array}$ & All Axes & Laser Interferometer & \\
\hline Squareness & All Planes & Laser Interferometer & $\begin{array}{l}\text { Measurement Along } \\
\text { Diagonals in Plane }\end{array}$ \\
\hline Roll & Hoxizontal Axes & Electronic Levels & \\
\hline Roll & Vertical Axis & $\begin{array}{l}\text { Offset Probe \& } \\
\text { Straightedges }\end{array}$ & \\
\hline
\end{tabular}




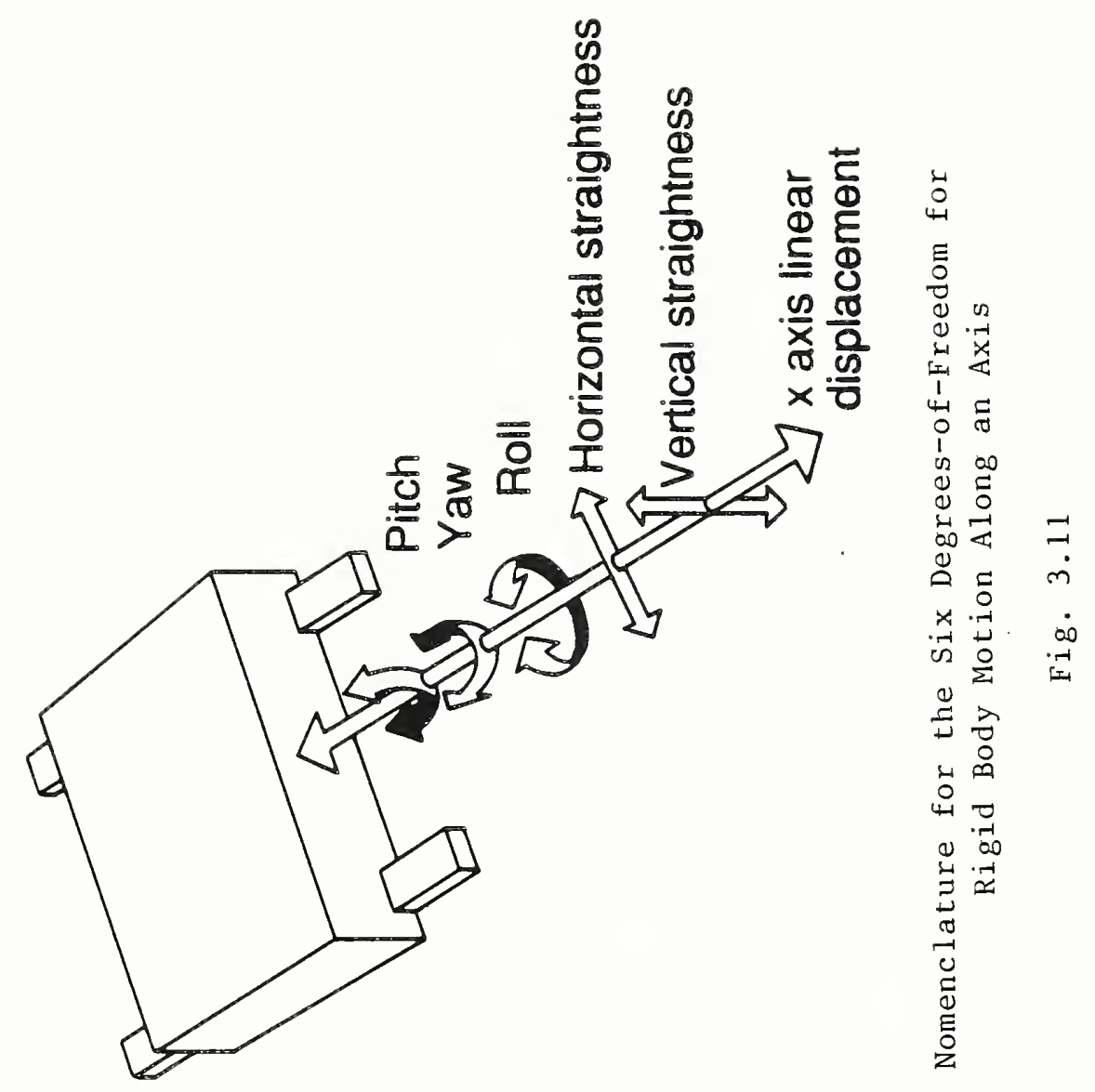




\section{3 .6 DATA ANALYSIS}

The position error data represents the combined effect of the geometric errors characteristic of the machine tool configuration and construction and the temperature distribution and resulting thermal distortion at the tool tip. As described previously, the thermal distortion most likely is characterized by an instantaneous temperature and a set of temperatures describing the prior thermal environment. This consideration identifies the requirement to evaluate the analysis techniques used to develop the position error compensation. Possible alternative analysis methods are described below. However, the basic approach will be discussed first.

a) Quasi-static conditions: For a very slowly changing thermal environment, it can be assumed that the temperature distribution results in insignificant position errors. In this case the basic approach to developing a compensation algorithm is to estimate statistically the position errors as a function of the machine tool axes positions. The resulting compensation would be estimated, in this case, using some form of regression analysis and would, most likely, result in a table look up scheme. Classical least-square analysis or a numerically-generated "weighted" regression analysis would be appropriate in this situation.

b) Dynamic conditions without Time-Lag: For a changing thermal environment without time-lag effects, the classical least-square analysis and the "weighted" regression analysis also apply. Donmez et al. [4], for instance, were quite successful in developing computational algorithms for real-time compensation for a CNC turning machine center. Their approach utilized high-order polynomials relating total position errors to the machine tool axis position and instantaneous temperatures monitored during the machining operation. Whereas a classical least-square regression analysis results in an 
analytical equation suitable for computation, the weighted regression analysis results in a table look up format.

c) Dynamic conditions with Time-Lag: For a changing thermal environment with time-lag effects, some form of active or dynamic compensation is required. The basis for considering time-lag effects is the nonequilibrium temperature conditions characteristic of machining operations. Since time is an additional parameter, some form of multivariable regression analysis is required to estimate the position errors resulting from thermal distortion. The approach taken to implement the compensation may be in the form of a postulated mathematical relationship using empirically derived constants [34], a computationally intensive scheme such as the General Method of Data Handling or GMDH modeling [37] or a table look up scheme based upon a multivariable regression model derived from a well-constructed data base of position errors and temperature time-histories.

In each of the above approaches, it will be necessary to establish the required time-lag and this, in turn, will establish the update rate for revising the machine tool compensation. Janeczko, for example, has indicated that an update rate of 10 seconds is appropriate for a horizontal machining center. 


\section{TEST PARTS AND INSPECTION RESULTS \\ C. Denver Lovett}

\subsection{GENERAL}

As stated earlier the ultimate goal of the PFMS is to implement a quality control system that assures a high level of product quality without depending on traditional product inspection methods to provide that quality assurance. The QIA objective is to develop a test that could evaluate the effectiveness of the QIA strategies in controlling machining variability and to measure the effectiveness of the in-process quality assurance. It is essential that a part of this evaluation include a baseline characterization of the machining performance of each machine, because the final conformance of the workpiece to its specifications ultimately tests the accuracy of the machine tool. As part of this evaluation process, the on-machine probing results obtained from selected test parts will be compared with the dimensional inspection results obtained from coordinate measuring machines for those same test parts.

\subsection{TEST PART SELECTION}

Two test parts were selected for the project. The horizontal machining center and the vertical machining center are being evaluated with the NAS 979 composite cutting test [38], and the turning center is being evaluated with the modified BAS test [39]. These test parts are shown in Fig. 4.1. Our primary purpose in both tests is to evaluate the accuracy and repeatability of the machine under loaded conditions. Other sections of the NAS 979 test also check the machine's dynamic stiffness and overshoot and undercut, which verify the dynamic response of the machine and the machine's control system, but these will not be tested initially. 


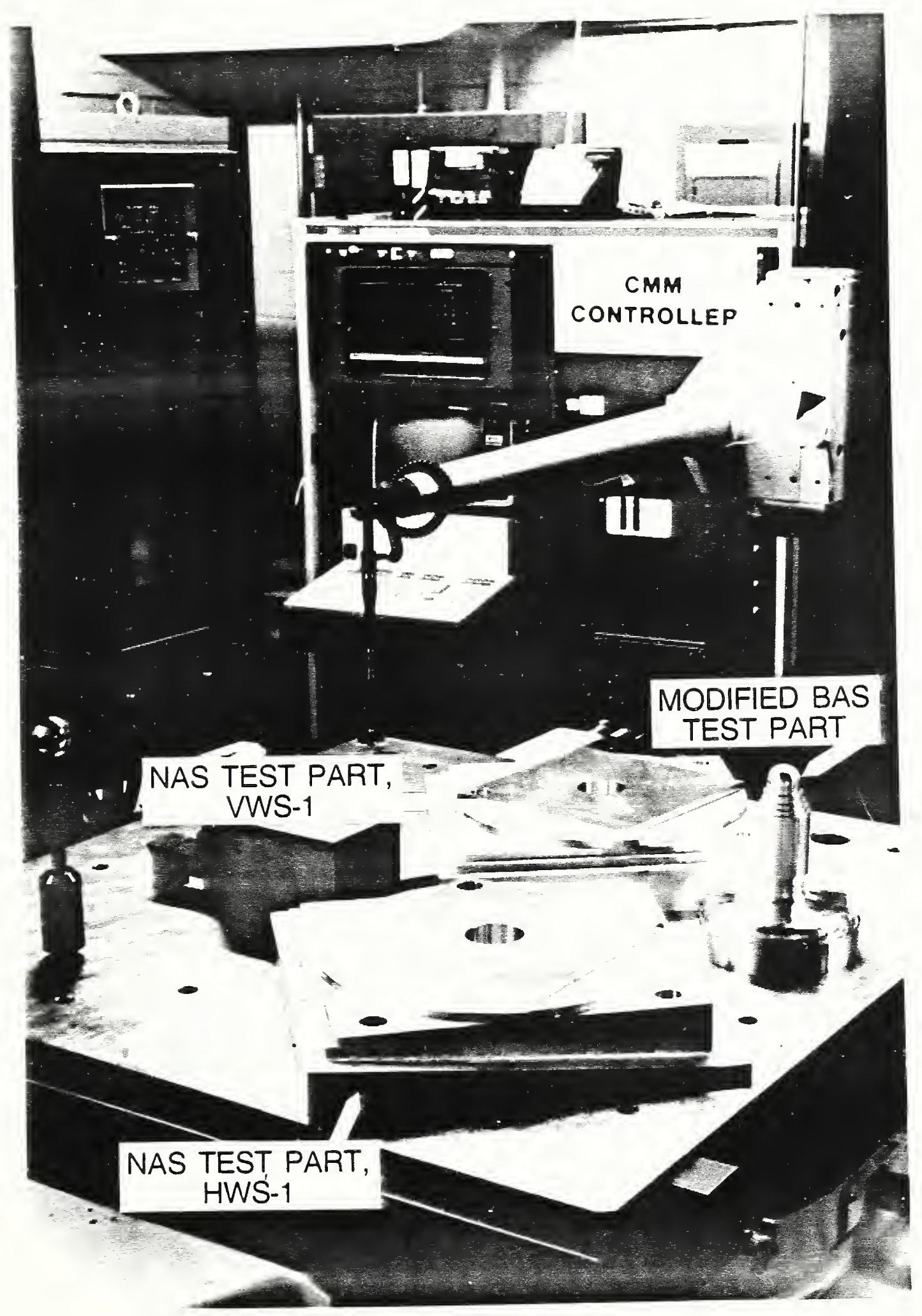

TEST PARTS

Fig. 4.1 


\subsection{FIXTURES AND BLANK PREPARATION}

Fixtures for mounting the test parts have been designed and fabricated. Six test-part blanks were prepared; four for the horizontal machining center and two for the vertical machining center.

\subsection{NC CODE FOR FIRST TEST PART AND INSPECTION}

The NC code has been written for all three machines. The first NAS test-part was machined on each of the two machining centers and the first modified BAS test-part was machined on the turning center. The inspection of these baseline NAS parts was performed with a coordinate measuring machine with nominal accuracy of 0.0001 inch. The parts are showr in Fig. 4.1. The VWS-1 part was machined on the Vertical Workstation and the HWS-1, on the Horizontal Workstation.

We are currently developing programs for the computer directed inspection of these parts. These baseline parts and all subsequently machined parts will be evaluated with the automated computer directed inspection system of the AMRF.

The inspection result for the two NAS test parts revealed good conformity to the tolerances that were specified in these tests with a few exceptions. Both parts, HWS-1 and VWS-1 failed the circular concentricity tolerance. The HWS-1 part also failed the diameter tolerance and its ramp failed to conform to the specified angularity tolerance.

Some of the inspection results of the NAS 979 part (HWS-1) are shown as follows: (1) the dimensions for the large square are shown in Figs. 4.2 and 4.3 , (2) the radii for the circle at twelve points are shown in Fig. 4.4, and (3) the dimensions for the small square are shown in Figs. 4.5 and 4.6. These figures are from an internal inspection sheet prepared by David Capparrelli [40]. 


\section{NAS 979 CUTTING TEST PART NUMBER HWS-1}

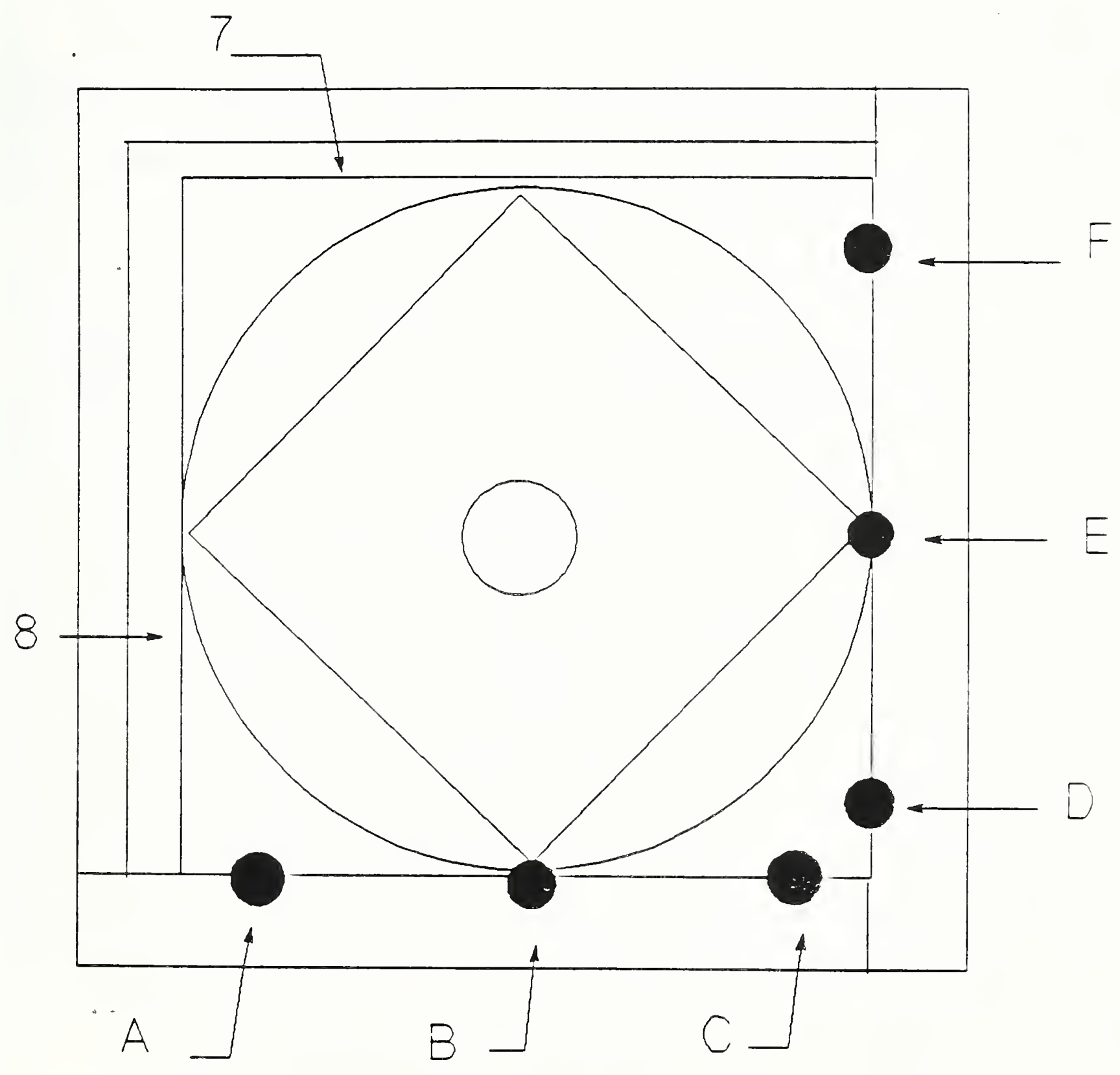

Figure 4.2 - Six Probing Points For Larger Square 


\section{NAS 979 CUTTING TEST PART NUMBER HWS-1}

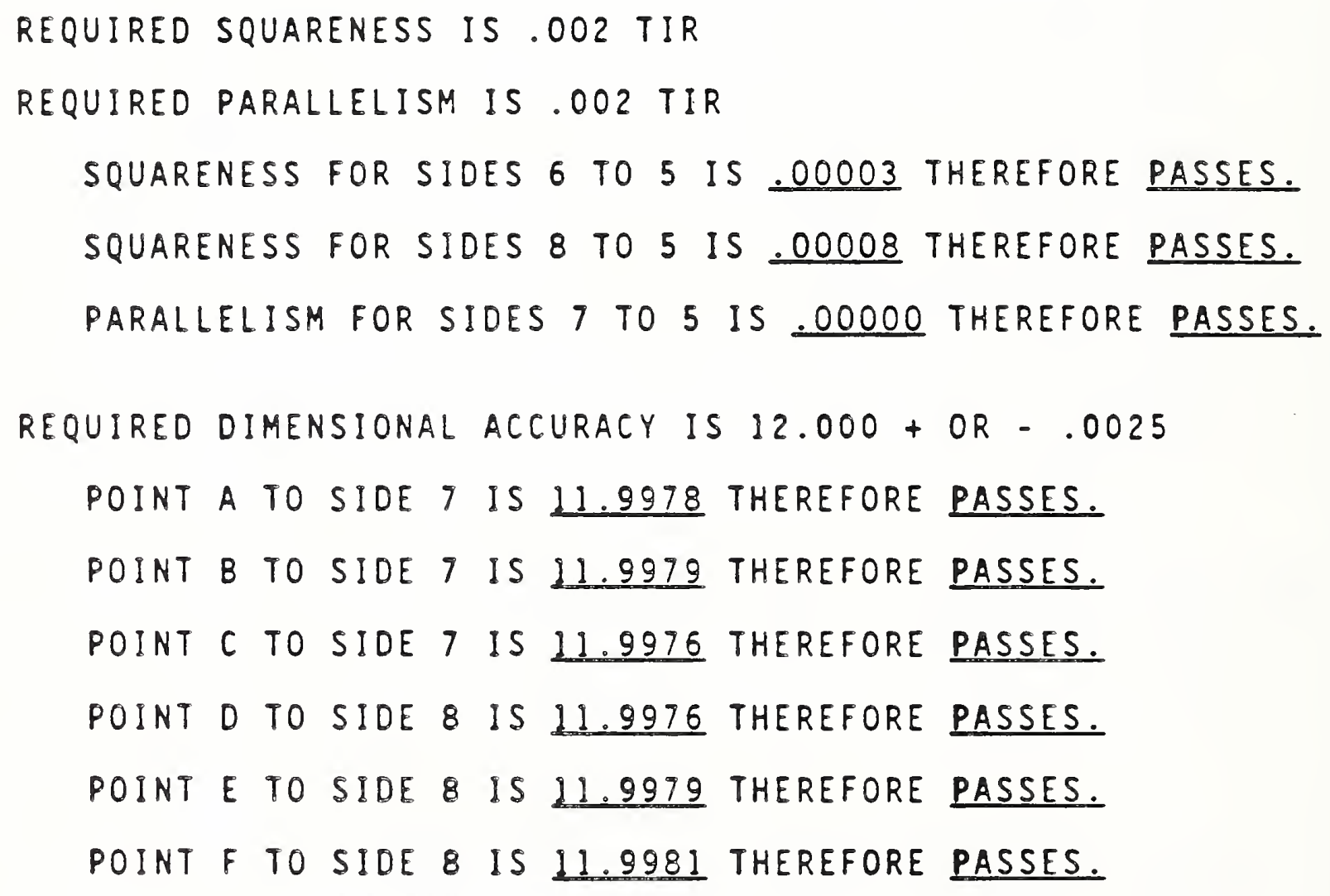

Figure 4.3-Measured Values of Length, Squareness and Parallelism for Large Square 


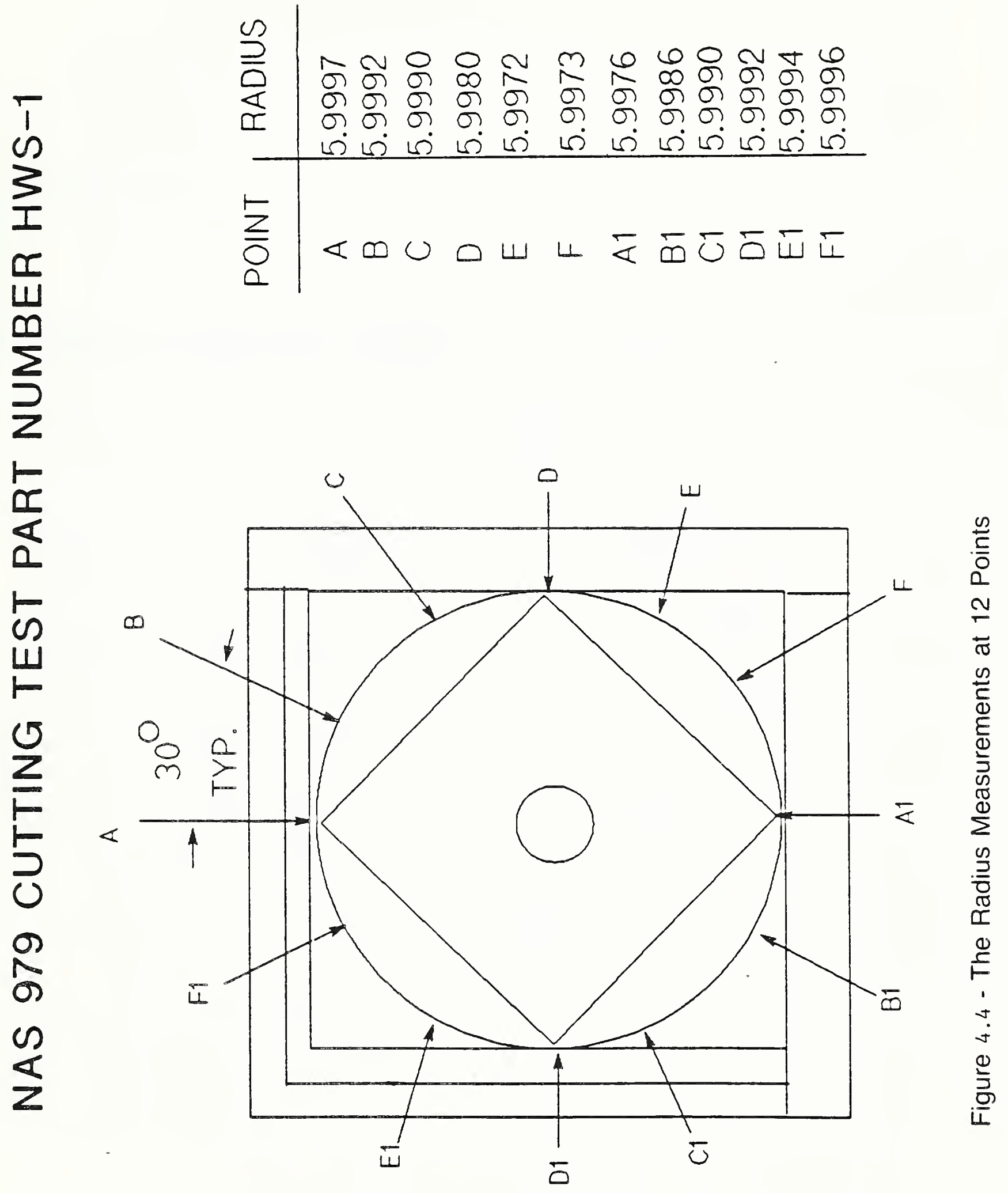




\section{NAS 979 CUTTING TEST PART NUMBER HWS-1}

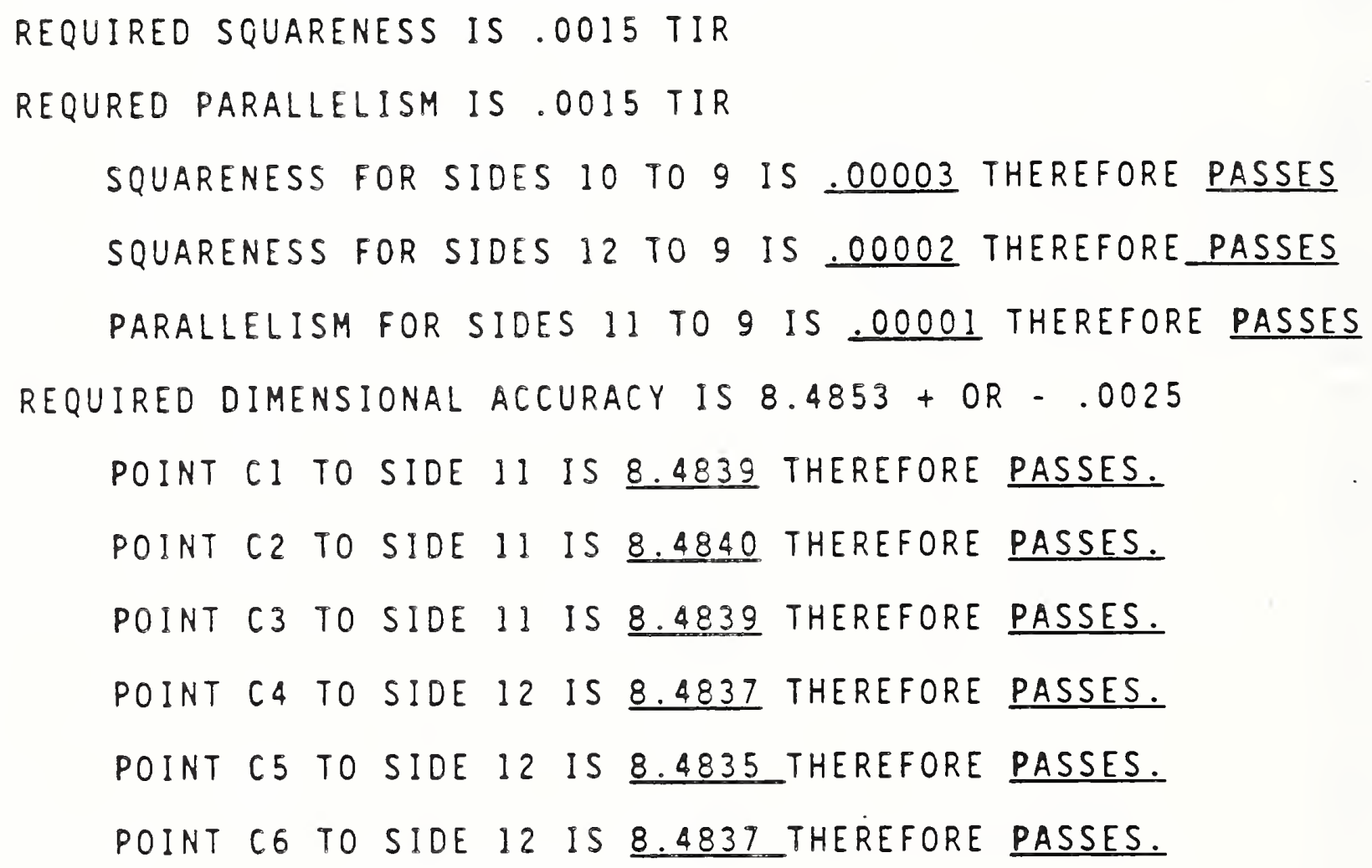

Figure 4.5 - Measured Values of Length, Squareness and Parallelism for Small Square 
NAS 979 CUTTING TEST PART NUMBER HWS -1

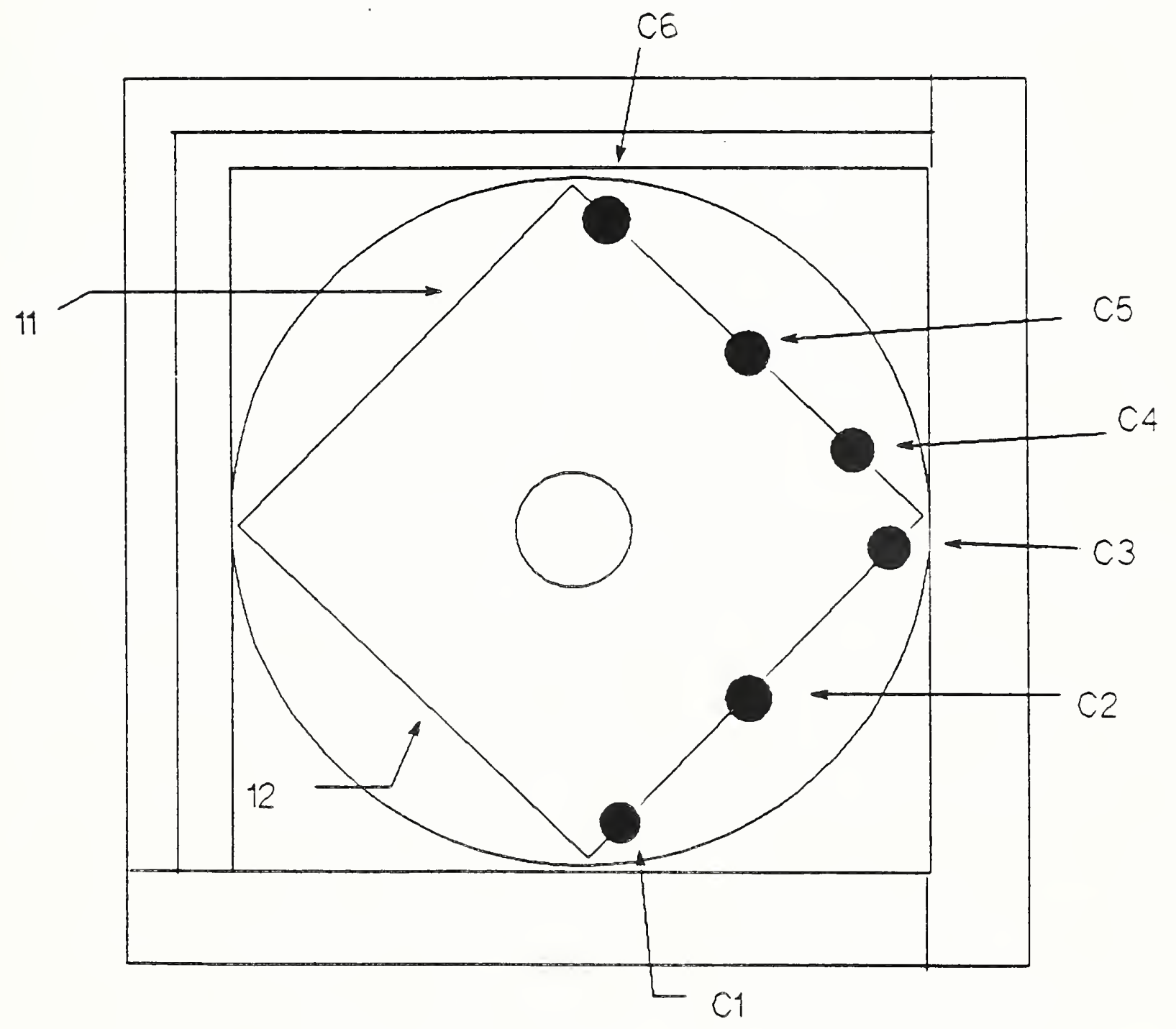

Figure 4.6 - Six Probing Points For Small Square 
Figure 4.2 clearly identifies sides 7 and 8 . Side 5 is opposite to side 7 and side 6 is opposite to side 8 . Points A, B, and C are located on side 5 and points $D, E$, and $F$ are located on side 6 . These points were selected for measuring distances between opposite sides of the square at the six locations shown in Fig. 4.2. As shown in Fig. 4.3, the six measured values to the reference sides 7 and 8 , all fall within the acceptance reference tolexance of \pm 0.0025 inch.

The inspection procedure for measuring squareness, parallelism, concentricity and ramp angle is described in the inspection sheets prepared by David Capparrelli [40].

The NAS 979 specification gives tolerance as low as \pm 0.0015 inch on some dimensions and as high as \pm 0.0025 inch on other dimensions. However, our goal is to maintain tolerance of \pm 0.001 inch for all dimensions. 


\title{
5. REAL-TIME ERROR CORRECTOR AND PROCESS-INTERMITTENT PROBING
}

\author{
K. W. Yee
}

\subsection{GENERAL}

Some error sources require immediate control actions in order to avoid unacceptable machining variability. The time-variant influence of temperature, vibration, and cutting forces, for example, sometimes dictates a hardware approach to error correction rather than a software approach. Shorter product cycle-time and demands to move from the traditional product inspection methods toward the active control of the machining process are additional reasons for developing an add-on hardware control feature that allows intermittent and active control of the machining process by an external computer. Existing on-machine probing is slow and time consuming; and the probing results are not easily transferrable to an external PC computer. These are problems that must be overcome, if the in-process quality assurance method is to replace the traditional inspection methods. These are realistic problems that are currently being addressed by the Real-Time Error Corrector (RTEC).

\subsection{CONCEPTUAL DESIGN OF RTEC}

The preliminary design for the RTEC is completed and its functions and design concepts are described below.

The RTEC is a hardware device that can be interfaced with the positional feedback elements (e.g., resolver, encoder, glass scale) for the axes of the machine tool. This device will unintrusively count the number of pulses in the signal from the feedback element, which provides the machine axes position to the machine tool controller (MTC). Based on this count, the RTEC will generate a position signal that is sent to the quality controller. Figure 5.1 shows the functional relationship of the RTEC to the other components in the system. 


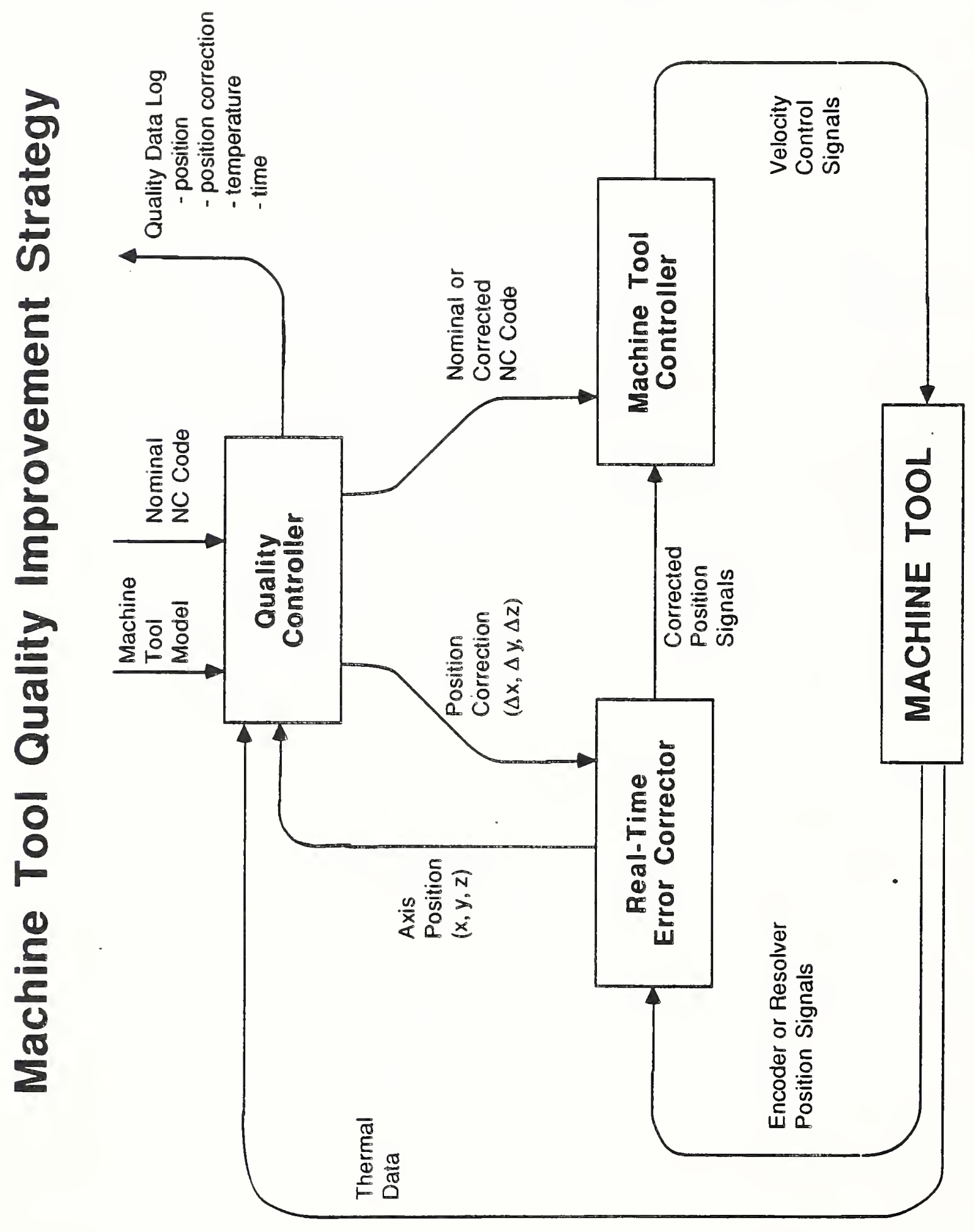


The RTEC will serve two functions: (1) during machining it will allow real-time error correction by modifying the machine tool axes position, (2) during process-intermittent gauging it will capture the axes position data in response to a touch probe "trip-point" and it will send that data to the quality controller. The RTEC will perform these functions without intrusion into the MTC and in a way that is invisible to the machine tool. The RTEC controls axis position by sightly modifying the signals from the feedback device to the MTC to make the axes go to a slightly different position than they would have without the correction applied. The quality controller has an empirically determined model that uses the axis position data and the temperature data to calculate a total correction factor. This total correction factor is based on both fixed geometric error and temperature-induced errors.

For process-intermittent gauging the RTEC is being designed to interface the quality controller to the probe measuring system. The design concept that is being implemented involves latching the axes position data in response to a probe "trip-point". The quality controller will download an inspection program to the MTC. This program will command the machine tool to rapidly move slightly beyond discrete points on the boundaries of a machined feature on the part. Each time the stylus of the probe passes the boundary by a small amount, the probe will trip and the RTEC will capture the axes position data at the instant the probe trips. Using this approach it is anticipated that the machine can be commanded to move the probe stylus at rates of 200 to $300 \mathrm{in} / \mathrm{min}$.

\subsection{IMPLEMENTATION}

The RTEC will be implemented on the vertical machining center (VMC), the turning center (TC) and a horizontal machining center (HMC). The VMC and the TC have GE-2000 controllers and both of these machines have glass scales as position feedback, which gives encoder type signals. The HMC has encoders. The RTEC will first be installed on the TC and then the VMC. 
The Real-Time Error Corrector is expected to contain a fast, singlechip microcomputer for each machine axis, which will both count the number of pulses in the position feed-back signal to keep track of absolute machine position and alter the position signals fed to the machine-tool controller to cause the machine to go to corrected positions. This altering of the positions will correspond to adding or dropping pulses from the "encoder" so that the machine axis moves further or stops sooner than ordinarily.

\subsection{TESTING}

The correction concept has been tested in hardware on the turning center. We have successfully moved the machine axis by a known amount (up to 10 milli-inches) plus and minus of the nominal position. The correction can be inserted with the axis moving in either direction. Corrections have been successful at slow feed rates typical of machining and at a rapid traverse speed of $275 \mathrm{in} / \mathrm{min}$. The velocity servo does not appear to be affected for this MTC. The resolution of the correction is 4 micrometers (approx. 160 microinches). The detailed design of the final hardware and correction software is underway. 


\title{
6. QUALITY ARCHITECTURE NETWORK
}

\author{
D. Blomquist
}

\subsection{GENERAL}

We are networking our QIA computers so that timely quality information is shared among the following elements of the QIA system: (1) quality monitors, (2) quality controllers, (3) inspection workstation, (4) CAD system and (5) the database. The network enables information to flow from the proper sources to the required destinations. The control loops that will require network service are shown in Figs. 2.2 and 2.6 , and key elements are described in Table 2.2. The network plan that we have started implementing is shown in Fig. 2.9. We will use two networks already in place, the AMRF network and the local Ethernet, and link thern together via a gateway to the VAX computer that serves the AMRF network. The AMRF Network has been in existence since 1983 and is discussed elsewhere [41].

\subsection{ETHERNET FEATURES}

This network provides flexibility by allowing communication among equipment from many different vendors. As long as the vendors' equipment operate within the ISO Open System Interconnect (OSI) Protocol, the equipment can communicate with other vendor's equipment over the network. The network implementation covers all seven layers of the OSI $[42,43]$. The first two layers are devoted to the physical link and the data link. These layers are connected by fiber optics. The fiber optics allows the Ethernet network to have a total length up to $3 \mathrm{~km}$. The application layers, five through seven are implemented in Xenix networking software. This software allows the quality monitor and the quality controller to access any file from the five file servers within the network. The five file servers have a storage of 650 Mbytes.

The Xenix networking software allows file protection and file sharing within the network. Both of these operations are transparent to the user 
of the file. Locking primitives are used to synchronize concurrent sharing of network files. Users at any quality monitor will be able to retrieve files created at any quality controller.

\subsection{IMPLEMENTATION}

The QIA project uses four of the fifty Ethernet workstations and one file server, although all five are available. When the network plan is fully implemented, the QIA system will link five quality monitors, three quality controllers, two inspection workstations, and a CAD system. Two of the quality monitors will be located in the metrology building at NIST, one in the shops and two in the sound building. 


\section{ULTRASONIC SENSOR \\ G.V. Blessing}

\subsection{GENERAL}

New developments in sensor technology are needed in order to achieve in-process quality assurance based on deterministic metrology and realtime sensors. A variety of sensors must be developed to measure the variability in machining. One example of this, a real-time sensor that can measure the relative magnitude of a machining process parameter, is the ultrasonic sensor for measuring surface finish. One of the real-time sensors that this QIA program will develop is a sensor that will produce an acoustic signal that contains information for evaluating surface roughness during the machining operation. This acoustic signal may contain other important information about the variability in the machining process. The conceptual design for this sensor is completed and is described below.

\subsection{CONCEPTUAL DESIGN}

An on-line ultrasonic sensor system is being developed to measure the surface finish or roughness of a turned part. The possibility of realtime monitoring during part fabrication in a machining center is also being considered. The purpose is two-fold: (1) to evaluate the surface roughness per se, and (2) to use that surface information to detect cutting-tool condition and possibly other machining parameters.

The approach being taken is to couple short bursts of ultrasound between the sensor and the part surface via a stream of the coolant/lubricating fluid existent in the cutting process. As such it is a noncontacting technique that can be applied to a rapidly turning part.

The same sensor is used to transmit and receive signals in a pulseecho mode of operation. The reflected echoes are a measure of the surface roughness, decreasing in amplitude with increasing roughness due to 
increased scattering. The sensor itself is constructed around a piezoelectric transducer that converts an electric excitation signal into an elastic wave pulse on transmission, and vice versa on reception. The wave pulses, typically less than one microsecond in duration, are generated at a nominal repetition rate of $1 \mathrm{kHz}$. The ultrasonic carrier frequency is in the megahertz region.

\subsection{IMPLEMENTATION AND TEST}

In our initial tests, a transducer operating at an ultrasonic frequency of $10 \mathrm{MHz}$ ( $150 \mu \mathrm{m}$ wavelength in the coupling fluid) was used, producing an insonified area of about $1.0 \mathrm{~mm}^{2}$ on the part surface. With such a wavelength and beam spot size much greater than the micrometer dimensions of the surface features, the sensor is operating in the mode of a scatterometer as opposed to a profilometer. The ultrasonic signature from an unknown surface may then be interpreted by a comparator technique, with reference to known surface signatures.

The results obtained on a $10 \mathrm{~cm}$ diameter aluminum cylinder mounted in the Monarch Metalist turning center have been encouraging. Two areas of the cylinder surface, with respective roughness average $\left(R_{a}\right)$ values of 0.6 and $4.6 \mu \mathrm{m}$ as determined by contacting stylus measurements, were studied under varying operating conditions. The received echoes (at a rate of one thousand per second) were amplified and passed through a detector stage to yield a proportional dc level output for recording purposes. With the surface stationary (Fig. 7.1a)), the relative echo amplitude of the rough section was $0.7 \mathrm{~dB}$ less than the smooth section. Upon rotation at 40 and then $500 \mathrm{rpm}$ (Fig. 7.1(b)), the relative difference in peak signal level remained rather constant. As the variation in the signal levels increased, the rough surface signal became more variable than the smooth surface signal. This was attributed to turbulence at the fluid-metal interface. Figure 7.1b illustrates these contrasting features to which microprocessing could be applied to correlate surface and signal features. 


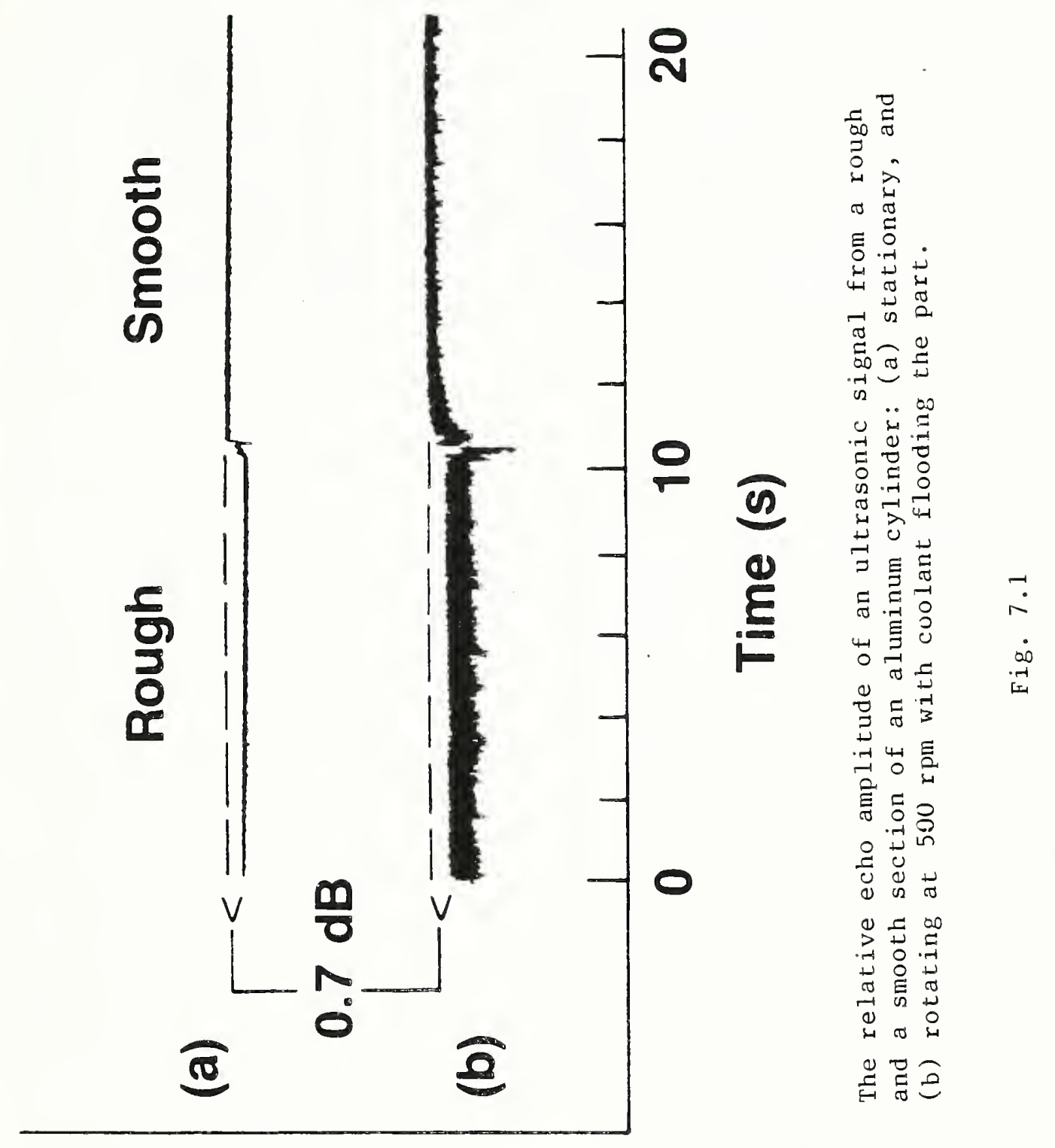

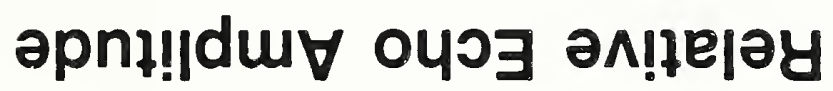




\subsection{FUTURE PLANS}

Future plans include improvements to the mechanical configuration of the sensor and increased ultrasonic sensitivity to fine surface features. We will try to mount the sensor unobtrusively on the tool turret of the turning machine and directly tap into the coolant line available on the turret as the source for our coupling fluid. We plan to take the received echo signals and pass them through an $A / D$ converter. Subsequent microprocessing of these echo signals will increase system sensitivity and eliminate extraneous echo signals such as those due to turbulence. Finally, we will increase the ultrasonic frequency with a goal of achieving a surface roughness resolution of better than $1 \mu \mathrm{m} \mathrm{R}_{\mathrm{a}}$. 


\section{VISUALIZATION OF INSPECTION RESULTS}

\section{H. T. Moncarz}

\subsection{GENERAL}

As quality assurance moves from the traditional post-process inspection methods toward in-process inspection methods, it is essential that efficient certification procedures are developed for evaluating the effectiveness of the in-process inspection results as compared with the traditional post-process inspection methods. Effective methods for correlating the on-machine probing results with the results obtained from the coordinate measuring machines need to be developed. The QIA approach is to use a quality monitor that relies heavily on graphical representation of quality information; thus giving the operator the capability to visualize and compare the many aspects of the inspection results. The main objective of this work is to facilitate the certification of the process by avoiding the burdensomeness of comparing large amounts numeric data taken from the two different inspection methods.

\subsection{DESIGN CONSIDERATIONS}

This task involves designing software and/or selecting software that will graphically emphasize the small difference obtained from two different irspection procedures. The program will read in a file that specifies the part's geometry and topology. This file may eventually be a PDES file. The visualization system will display a three-dimensional representation of the part on the Quality monitor screen. This system will allow the operator to rotate, translate and size the part in any manner desired, using a menu system with a mouse.

This program will provide two types of procedures. The first procedure will allow the operator to select an entity or a group of entities that are associated with the ideal part or with the actual part, based on inspection data. An example of these entities could include 
features from the ideal part, the set of all probe points, individual probing points picked by the mouse, and fitted surfaces. The user interface will be designed to include a mechanism which would allow the user to enter his preferred selection algorithm.

The second type of procedure would allow the operator to select the type of operation to be performed on a particular entity, which was previously selected. One operation would include enhancements to exaggerate the difference between actual measurements and the ideal part. Graphical facilities will allow the user to visualize these differences. Another operation would include statistical algorithms, for example, to fit points measured to the types of surfaces which they ideally represent.

\subsection{IMPLEMENTATION AND TESTING}

One module of the computer progam is completed. It is implemented on the IRIS Silicon Graphics computer in the $C$ programming language. This module can read an arbitrary AMRF Part Model data file and display a three dimension wire frame representation of the part on the screen. The viewing attributes can be selected before the program is started. This module will become one component of the completed program. 


\section{REFERENCES}

[1] Blaedel, K.L., "Error Reduction," in Technology of Machine Tools: Machine Tool Accuracy, Vol. 5 (Lawrence Livermore National Laboratory, Livermore, CA, 1980).

[2] Simpson, J.A., "The National Bureau of Standards Approach to Quality," Test \& Measurement World $\underline{3}, 38$ (Dec. 1983).

[3] ANSI B46.1-1985, "Surface Texture, Roughness, Waviness, and Lay" (ASME, New York, 1985).

[4] Donmez, M.A.; Blomquist, D.S.; Hocken, R.J.; Liu, C.R.; Barash, M.M., "A General Methodology for Machine Tool Accuracy Enhancement by Error Compensation," Prec. Eng. 4, 187 (1986).

[5] Donmez, M.A.; Liu, C.R.; Barash, M.M., "A Generalized Mathematical Model for Machine-Tool Errors," in Modeling. Sensing, and Control of Manufacturing Processes, BK.No.H00370, K. Srinivasan, D.L.E. Hardt, and R. Komanduri, eds. (ASME, New York, 1987).

[6] Donmez, M.A.; Lee, K.; Liu, C.R.; Barash, M.M., "A Real-Time Error Compensation System for a Computerized Numerical Control Turning Center," Proc. IEEE Int. Conf. on Robotics and Automation (IEEE Computer Soc., Washington, D.C., 1986).

[7] Machining Data Handbook, 3rd Edition (Metcut Res. Assocs., Cincinnati, OH, 1980).

[8] Evans, L.; Yee, K.W., "Alternatives for Sensing Tool Wear in Peripheral and Slot End-Milling," SME Technical Paper MS851001 (Soc. Manuf. Engs., Dearborn, MI, 1985).

[9] Kisler and Kennametal Force Dynamometer.

[10] Blessing, G.V.; Eitzen, D.G., "Surface Roughness Sensed by Ultrasound," Surface Topography 1 , 253 (1988).

[11] Somerville, L., Renishaw Corporation (private communication).

[12] Moncarz, H.T.; Vorburger, T.V., Implementation of the Surface Roughness Instrument (SRI) Controller, NISTIR 88-3794 (U.S. Dept. of Commerce, NIST, 1988).

[13] Zimmerman, J.; Vorburger, T.V.; Moncarz, H., "Automated Optical Roughness Inspection", in Optical Testing and Metrology II, Proc. SPIE. 954,252 (1988). 
[14] Garbini, J.L.; Jorgensen, J.E.; Down, R.A.; Kow, S.P., "Fringe Field Capacitive Profilometry," Surface Topography 1, 99 (1988).

[15] Lieberman, A.G.; Vorburger, T.V.; Giauque, C.H.W.; Resnick, R.; Rose, J., "Capacitance versus Stylus Measurements of Surface Roughness", Surface Topography 1, 315 (1988).

[16] Kohno, T.; Ozawa, N.; Meyamoto, K.; Musha, T., "Practical Non-Contact Surface Measuring Instrument with One Nanometer Resolution", Prec. Eng. I, 231 (1985).

[17] Brodmann, R.; Smilga, W., "Evaluation of a Commercial Microtopography Sensor", Proc. SPIE 802, 165 (1987).

[18] Proc. of the ASME/NSF Workshop on Mechanical Tolerancing, (American Society of Mechanical Engineers, New York, 1989) to be published.

[19] ISO/TC184/SC4/N32, STEP Physical File Structure, SC4/WG1, Document number N219, May 1988.

[20] Kramer, T.R., Process Plan Expression. Generation, and Enhancement for the Vertical Workstation Milling Machine in the AMRF at NBS, NBSIR 87-3678 (NIST, Gaithersburg, MD, 1987).

[21] "Interchangeable Variable Block Data Format for Positioning, Contouring, and Contouring/Positioning Numerically Controlled Machines", (Electronics Industries Association, February 1979).

[22] ANSI X-3 135, 1986 "Data Base Language-SQL", (American National Standards Institute, Inc., October 1986).

[23] Bandy, H.T.; Carew, V.E., Jr.; Boudreaux, J.C., An AMPLE Version 0.1 Prototype: The HWS Implementation, NBSIR 88-3770 (NIST, Gaithersburg, MD, 1988).

[24] Boudreaux, J.C., "Problem Solving and the Evolution of Programming Languages," in The Role of Language in Problem Solving-1, R. Jernigan, B.W. Hamill, and D.M. Weintraub, eds. (North-Holland, Amsterdam, 1985), pp. 103-126.

[25] Boudreaux, J.C., "AMPLE: A Programming Language Environment for Automated Manufacturing, " in The Role of Language in Problem Solving-2, J.C. Boudreaux, B. Hamill, and R. Jernigan, eds. (NorthHolland, Amsterdam, 1987).

[26] Boudreaux, J.C., The AMPLE Project, NBSIR 86-3496 (NIST, Gaithersburg, MD, 1986).

[27] Boudreaux, J.C., OED: Object-Oriented Editor, NBSIR 86-3530 (NIST, Gaithersburg, MD, 1986). 
[28] Boudreaux, J.C., "Requirements for Global Programming Languages," in Proc. of the Symposium on Manufacturing Application Languages, MAPL $\underline{88}, 107$ (1988).

[29] van Wijk, J.J., On New Types of Solid Models and Their Visualization with Ray Tracing (Delft University Press, 1986), Chap. 3 and 4.

[30] Sowa, J.F., Conceptual Structures: Information Processing in Mind and Machine (Addison-Wesley, 1984), Chap. 3.

[31] Staley, S.M.; Boudreaux, J.C., "Programming Language Environments for CIM," in Proceedings of PROCIM 88.

[32] Jackson, R.H.F.; Jones, A.W.T., "An Architecture for Decision Making in the Factory of the Future," Interfaces 17 (6), 15 (1987).

[33] Jones, A.; McLean, C., "A Proposed Hierarchical Control Model for Automated Manufacturing Systems," J. Manuf. Systems 5 , 15 (1986).

[34] Janeczko J., "Machine Tool Thermal Distortion Compensation", Proc. 4 th Biennial International Machine Tool Technology Conference, September 7-14, 1988, Session 17, (National Machine Tool Builders Association, McLean, VA) pp. 7-167 to 7-181.

[35] Paul, R.P., Robot Manipulators: Mathematics, Programming, and Control (The MIT Press, Cambridge, MA, 1981).

[36] Zhang, G.; Veale, R.; Charlton T.; Borchardt B.; Hocken R., "Error Compensation of Coordinate Measuring Machines," CIRP 34, 445 (1985).

[37] Lee, S.K.; Barash, M.M., Accuracy Improvement of a CNC Machining Center by Using a Touch Probe and a Metrology Pallet, Final Report Vol. 6 (School of Industrial Engineering, Purdue University, West Lafayette, IN, 1986).

[38] National Aerospace Standard, NAS 979. Uniform Cutting Tests - NAS Series Metal Cutting Equipment Specifications (National Standards Association, Washington, D.C., 1969).

[39] BAS Machine Tests (Sveriges MeKanf8bound, Box 5506, S-114 85 Stockholm).

[40] Capparrelli, D., "Measurement Results for the NAS 979 Test Part (HWS-1)", (Internal Inspection Sheets) (NIST, Gaithersburg, MD, 1988).

[41] Rybszynski, S., AMRF Network Communication, NBSIR 88-3816 (NIST, Gaithersburg, MD, 1988). 
[42] IsO 8073 - "Information Processing Systems - Open System Interconnection - Connection Oriented Transport Protocol Specifications" (International Standards Organization, 1986).

[43] IEEE 802.3, "Standards for Local Area Networks: Carrieri Sense Multiple Access with Collision Detection" (1985), Supplements (1988). 
BIBLIOGRAPHIC DATA

SHEET (See instructions)

4. TITLE AND SUBTITLE

Progress Report of The Quality in Automation Project for FY88

\section{AUTHOR(S)}

Edited by $C$. Denver Lovett

6. PERFORMING ORGANIZATION (If joint or other than NBS. see instructions)

7. Contract Grant No.

NATIONAL BUREAU OF STANDARDS U.S. DEPARTMENT OF COMMERCE GAITHERSBURG, MD 20899

Document describes a computer program: SF-185, FIPS Software Summary, is attached.

11. ABSTRACT (A 200-word or less factual summory of most significant information. If document includes a significant bibliography or literature survey. mention it here)

This document describes a quality control architecture that uses real-time sensing, deterministic metrology methods, machine tool characterization, process-intermittent gauging, and process certification to control the machining process, with the objective of reducing the reliance on traditional post-process inspections methods and moving toward greater reliance on in-process verification. This Quality In Automation project is developing a computer-based system that includes real-time sensors, a quality database, quality monitors, quality controllers, an inspection station, and a communication network, which allows data to flow among components of the quality system. The quality architecture includes control loops such as post-processing characterization, pre-processing characterization, process intermittent gauging and real-time sensing. The sensors for temperature, force and ultrasonic may result in a command signal to the machine tool controller to alter its pre-programmed positions, to change its manipulative variables (speed and feed) and to modify the NC code. The corrective action will depend on the time varying nature of the errors and the predictions derived from the analysis models. A strategy for allocating the corrective action to a real-time error corrector or a software module that revises the process plan and the NC code is being developed. The machining of standard work pieces (NAS and modified BAS) and nachine tool characterization serves as a major component in certifying the machining process.

12. KEY WORDS (Six to twelve entries: alphabeticol order: capitalize only proper nomes; and separate key words by semicolons) controller; force; inspection; machine tool; monitor; process certification; process-intermittent gauging; quality; real-time control; sensors; temperature; ultrasonic

13. AVAILABILITY

$[\bar{X}$ Unlimited

For Official Distritution. Do Not Release to NTIS

Order From Superintendent of Documents, U.S. Government Printing Office, Washıngton. D.C. 20402.

14. NO. OF PRINTED PAGES

$$
107
$$

15. Price

Order From National Technical Information Service (NTIS), Springfield, VA. 2216I 
.

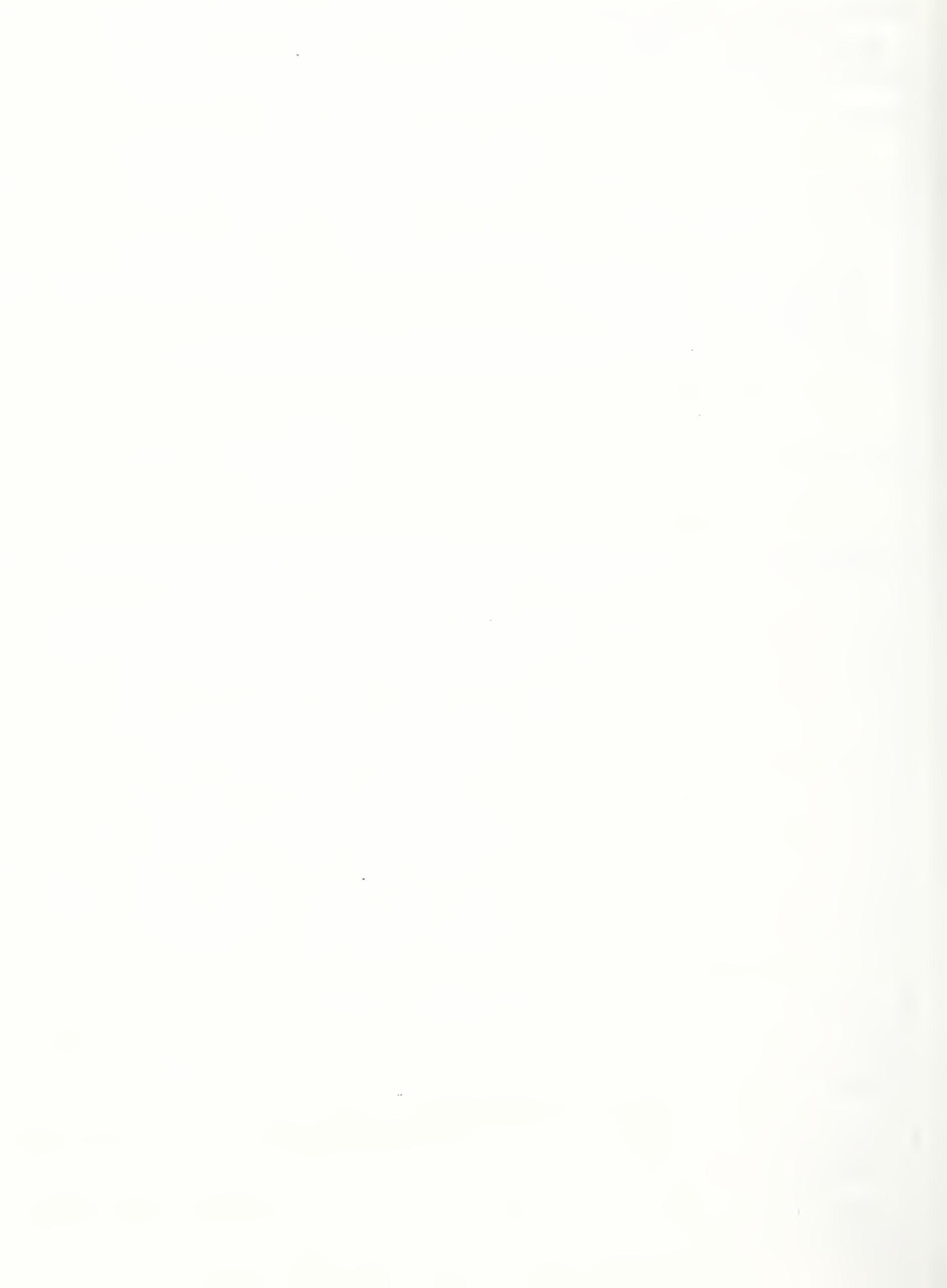



